\title{
Intrusion Detection System in Software-Defined Networks Using Machine Learning and Deep Learning Techniques -A Comprehensive Survey
}

\author{
Md. Rayhan Ahmed, Salekul Islam, Senior Member, IEEE, Swakkhar Shatabda, A. K. M. Muzahidul \\ Islam, Senior Member, IEEE, Md. Towhidul Islam Robin
}

\begin{abstract}
At present, the Internet is facing numerous attacks of different kinds that put its data at risk. The safety of information within the network is, therefore, a significant concern. In order to prevent the loss of incredibly valuable information, the Intrusion Detection System (IDS) was developed to recognize the outbreak of a stream of attacks and notify the network system administrator providing network security. IDS is an extrapolative model used to detect network traffic as routine or attack. Software-Defined Networks (SDN) is a revolutionary paradigm that isolates the control plane from the data plane, transforming the concept of a software-driven network. Through this data and control plane separation, SDN provides us the opportunity to create a manageable and programmable network, allowing applications in the top plane to access physical devices via the controller. The controller functioning inside the control plane executes network modules and establishes flow rules to forward packets in the switches residing in the data plane. Cyber attackers target the SDN controller to subdue the control plane, which is considered the brain of the SDN, providing a plethora of functionalities such as regulating flow control to switches or routers in the data plane below via southbound Application Programming Interfaces (APIs) and business and application logic in the application plane above via northbound APIs to implement sophisticated networks. However, the control plane becomes a tempting prospect for security attacks from adversaries because of its centralization feature. This paper includes an in-depth overview of the notable published articles from 2015 to 2021 that used Machine Learning (ML) and Deep Learning (DL) techniques to construct an IDS solution to provide security for SDN. We also present two detailed taxonomic studies regarding IDS, and ML-DL techniques based on their learning categories, exploring various IDS solutions to secure the SDN paradigm. We have also conducted brief research on a few benchmark datasets used to construct IDS in the SDN paradigm. To conclude the survey, we provide a discussion that sheds light on continuous challenges and IDS issues for SDN security.
\end{abstract}

Index Terms - Intrusion Detection System, Software Defined Networks, Machine Learning, Deep Learning, Network Security, Attacks.

\section{INTRODUCTION}

Network security is now one of the most significant concerns with the network's explosive development because it directly influences the interests of the nation, the companies, and the individuals. Modern IDS[1] in the network needs to cope with a substantial quantity of data being generated every second due to the swift expansion of information technology. The internet progression and the fast exchange of data brings the threat of increasing cyber-attacks targeting governments and commercial enterprises worldwide at a rapid rate. In response to that threat, the need to develop an intrusion detection system has been developed and intensified. IDS continuously monitors the network by creating a traffic pattern that enables itself to detect traffic behavior or patterns that deviate from the normal pattern. Continuous irregular traffic is deemed a threat that can eventually turn into an attack. IDS examines the network traffic records on computer networks to mark risky events and actions and warns when such an activity is identified. IDS also assists by detecting external attacks in the network.

According to [2], the worldwide market size for SDN is estimated to rise from USD 13.7 billion in 2020 to USD 32.7 billion by 2025 . The transpiring SDN architecture [3] provides more flexibility and control than traditional network architecture by splitting the data plane and the control plane in the SDN, hence providing more programmability and flexibility. The significant difference of SDNs with the standard hardwarecentric network paradigm is their functionality that varies according to their architecture. In a traditional hardware-based network, it is challenging to deploy new protocols and required services since many switches are required to be updated or changed with manual configuration, which is error-prone and sluggish. In contrast, in SDN, it is configured automatically along with a centralized authentication process. SDN's improved performance is based on dynamic global control and cross-layer information, whereas a traditional network is static with limited information within it [4]. Nevertheless, it is necessary to preserve a satisfactory security level with the versatility and manageability of SDN architecture. In addition to the classical IDS and Intrusion Prevention System (IPS), the IDS, incorporated with the SDN paradigm provides an additional security level due to the unique programmability feature of SDN.

With the rapid development of ML and DL-based applications in numerous network paradigms, IDS based on ML-DL is gaining much popularity for automated threat and intrusion detection within a network. Supervised and Unsupervised Learning schemes of ML and DL methods enable the IDS to distinguish both known and unidentified attacks by training the model with improved accuracy. In the literature, a number of IDSs are developed in the SDN paradigm by adopting various ML and DL algorithms. However, there is a shortage of in-depth, comprehensive survey that summarizes ML and DL-based recent development of IDS within the SDN that maps each study to a specific IDS domain. Table I presents a comparative analysis of 
this survey with previous surveys. We believe it is really important for a study covering IDS solutions in SDN to provide a detailed study of various IDS mechanisms.

To detect and prevent attacks in SDN, a taxonomic study of IDS can play a pivotal role for a developer to design a robust ML-DL-based IDS solution for the SDN paradigm by providing several IDS domain knowledge about the anomaly-based, misuse-based, host-based, and network-based IDS mechanisms, covering statistical analysis, log-data analysis, flow rules matching, expert-system development, pattern matching, packet inspection, session data analysis, and time-series-based subdomains. One of the significant factors in developing any ML-DL-based IDS in SDN is the appropriate choice of datasets. There is an apparent lack of study of the datasets used in the MLDL-based IDS-SDN research in the surveys mentioned above. Ref. [5], [6] provides a brief study about some of the commonly used datasets in developing IDS solutions. However, those surveys are not specific to providing solutions for SDN, neither provide a comparative summary among those datasets by differentiating each dataset from other in terms of their data collection format, labeling, type of addressing attacks, number of features, utilized network and topology, etc.; instead, those are very generic, leaning towards the traditional network-based IDS solution. Besides no other survey paper, reviews shallow ML, DL, Reinforcement Learning (RL), and Ensemble Learning (EL) mechanisms specific to provide IDS solutions in SDN in a single survey paper. Some notable surveys [6]-[9] provide a state-ofthe-art review of ML-DL-based IDS. However, those are leaning towards the generic network-based IDS solution, not specific to SDN, which is the primary field of this study. Therefore, this survey paper aims to review the state-of-the-art shallow MLbased, DL-based as well as RL-based and ensemble models for developing IDS in SDN architecture over the last six years following a taxonomic structure. The major contributions of this paper are enumerated below:

\begin{tabular}{|c|c|c|c|c|c|c|c|c|c|c|c|c|}
\hline \multicolumn{13}{|c|}{ TABLE I } \\
\hline \multicolumn{13}{|c|}{ COMPARISON WITH OTHER SURVEYS ( $\checkmark$ : ADDRESSED, X: NOT ADDRESSED) } \\
\hline \multirow[b]{2}{*}{ Ref. } & \multirow[b]{2}{*}{ Year } & \multirow{2}{*}{$\begin{array}{l}\text { Discussion of } \\
\text { SDN } \\
\text { architecture }\end{array}$} & \multirow{2}{*}{$\begin{array}{l}\text { Discussion of } \\
\text { OpenFlow } \\
\text { procedure }\end{array}$} & \multirow{2}{*}{$\begin{array}{c}\text { IDS-based } \\
\text { taxonomic } \\
\text { study }\end{array}$} & \multirow{2}{*}{$\begin{array}{l}\text { ML-DL- } \\
\text { based } \\
\text { taxonomic } \\
\text { study }\end{array}$} & \multirow{2}{*}{$\begin{array}{l}\text { Number of } \\
\text { reviewed papers } \\
\text { using ML and } \\
\text { DL to develop } \\
\text { IDS solution } \\
\text { (SDN-specific) }\end{array}$} & \multirow{2}{*}{$\begin{array}{c}\text { Study of } \\
\text { datasets in } \\
\text { the } \\
\text { reviewed } \\
\text { papers }\end{array}$} & \multicolumn{5}{|c|}{ AI Based Approaches for SDN-based IDS } \\
\hline & & & & & & & & $\begin{array}{c}\text { Shallow } \\
\text { ML }\end{array}$ & $\begin{array}{c}\text { Deep } \\
\text { Learning }\end{array}$ & $\begin{array}{l}\text { Reinforcement } \\
\text { Learning }\end{array}$ & $\begin{array}{c}\text { Hybrid } \\
\text { Mechanism }\end{array}$ & $\begin{array}{l}\text { Ensemble } \\
\text { Learning }\end{array}$ \\
\hline$[10]$ & 2017 & $\checkmark$ & $\mathbf{x}$ & $\checkmark$ & $\checkmark$ & 5 & $\mathbf{x}$ & $\checkmark$ & $\checkmark$ & $\mathbf{x}$ & $\mathbf{x}$ & $\mathbf{x}$ \\
\hline [7] & 2018 & $\mathbf{x}$ & $\mathbf{x}$ & $\mathbf{x}$ & $\mathbf{x}$ & Not Applicable & $\mathbf{x}$ & $\checkmark$ & $\mathbf{x}$ & $\mathbf{x}$ & $\checkmark$ & $\mathbf{x}$ \\
\hline$[11]$ & 2018 & $\checkmark$ & $\checkmark$ & $\mathbf{x}$ & $\checkmark$ & 16 & $\mathbf{x}$ & $\checkmark$ & $\checkmark$ & $\mathbf{x}$ & $\mathbf{x}$ & $\mathbf{x}$ \\
\hline [6] & 2019 & $\mathbf{x}$ & $\mathbf{x}$ & $\checkmark$ & $\checkmark$ & Not Applicable & $\mathbf{x}$ & $\checkmark$ & $\checkmark$ & $\mathbf{x}$ & $\checkmark$ & $\mathbf{x}$ \\
\hline$[12]$ & 2020 & $\checkmark$ & $\mathbf{x}$ & $\mathbf{x}$ & $\mathbf{x}$ & 17 & $\mathbf{x}$ & $\checkmark$ & $\checkmark$ & $\mathbf{x}$ & $\mathbf{x}$ & $\mathbf{x}$ \\
\hline$[13]$ & 2020 & $\checkmark$ & $\mathbf{x}$ & $\mathbf{x}$ & $\checkmark$ & 13 & $\mathbf{x}$ & $\checkmark$ & $\checkmark$ & $\mathbf{x}$ & $\checkmark$ & $\mathbf{x}$ \\
\hline$[14]$ & 2020 & $\checkmark$ & $\mathbf{x}$ & $\mathbf{x}$ & $\mathbf{x}$ & 30 & $\mathbf{x}$ & $\checkmark$ & $\checkmark$ & $\mathbf{x}$ & $\mathbf{x}$ & $\mathbf{x}$ \\
\hline$[15]$ & 2021 & $\checkmark$ & $\checkmark$ & $\mathbf{x}$ & $\mathbf{x}$ & 9 & $\mathbf{x}$ & $\checkmark$ & $\mathbf{x}$ & $\mathbf{x}$ & $\mathbf{x}$ & $\mathbf{x}$ \\
\hline [5] & 2021 & $\mathbf{x}$ & $\mathbf{x}$ & $\mathbf{x}$ & $\mathbf{x}$ & 4 & $\checkmark$ & $\mathbf{x}$ & $\checkmark$ & $\mathbf{x}$ & $\checkmark$ & $\mathbf{x}$ \\
\hline$[16]$ & 2021 & $\checkmark$ & $\mathbf{x}$ & $\mathbf{x}$ & $\checkmark$ & 79 & $\mathbf{x}$ & $\checkmark$ & $\checkmark$ & $\mathbf{x}$ & $\checkmark$ & $\checkmark$ \\
\hline [9] & 2021 & $\mathbf{x}$ & $\mathbf{x}$ & $\mathbf{x}$ & $\checkmark$ & Not Applicable & $\checkmark$ & $\checkmark$ & $\checkmark$ & $\mathbf{x}$ & $\mathbf{x}$ & $\mathbf{x}$ \\
\hline $\begin{array}{l}\text { This } \\
\text { Paper }\end{array}$ & - & $\checkmark$ & $\checkmark$ & $\checkmark$ & $\checkmark$ & 92 & $\checkmark$ & $\checkmark$ & $\checkmark$ & $\checkmark$ & $\checkmark$ & $\checkmark$ \\
\hline
\end{tabular}

- A comprehensive survey of existing IDS in SDN that use different ML and DL techniques is carried out. The existing works are then broadly categorized into ML, DL, RL and ensemble-based models. In each category, the existing solutions are compared based on the algorithms for building IDS solution, used dataset, core feature selection, SDN controller, and the attack classification type along with their names.

- Two taxonomies - taxonomy of IDS and taxonomy of ML-DL algorithms utilized in IDS - are developed. Using these two taxonomies summary and comparison of existing IDS solutions in SDN are also presented.

- The available benchmark datasets used to construct IDS in the SDN paradigm are reviewed and a comparative study is presented among those by highlighting their metadata, attack types, features, format, label, network, topology, and class balance issue also the reviewed papers are categorized along with their model evaluation metrics.

- Open issues and challenges in designing ML-DL-based IDS for SDN are also identified.

The organization of this survey is visually presented in Fig.1. Rest of the paper is organized as follows: Section II discusses about SDN, OpenFlow (OF) architecture, and various applications of SDN. Section III provides a taxonomic study of IDS. In section IV, we provide a taxonomic discussion about the AI-based IDS approaches that. In section V, we discuss about some frequently used ML-based IDS approach in SDN and review existing literature adopting this ML-based IDS approach. Section VI provides a literature review of some of the frequently used DL-based IDS approach in SDN, along with a brief study of the leveraged DL-based algorithms. In section VII, we review hybrid mechanism-based IDS approaches in SDN. In section VIII, we review Ensemble-based IDS approaches in SDN. Section IX provides taxonomic summary of all the reviewed articles of this survey. Section X provides a brief study of the .

.


frequently used datasets in IDS research along with their attack mechanism. In section XI, we discuss some of the research issues and underlying challenges in developing IDS solutions for SDN. Finally, we conclude the survey in section XII. The acronyms that will be followed throughout the paper is listed in Table II.

TABLE II

LIST OF SOME IMPORTANT ACRONYMS

\begin{tabular}{|c|c|}
\hline IDS & Intrusion Detection System \\
\hline SDN & Software Defined Networks \\
\hline ML & Machine Learning \\
\hline DL & Deep Learning \\
\hline IPS & Intrusion Prevention System \\
\hline RL & Reinforcement Learning \\
\hline OF & OpenFlow \\
\hline VoIP & Voice over Internet Protocol \\
\hline ICMP & Internet Control Message Protocol \\
\hline МPTCP & Multi-Path Transmission Control Protocol \\
\hline SSH & Secure Shell Protocol \\
\hline IMAP & Internet Message Access Protocol \\
\hline NFV & Network Function Virtualization \\
\hline DBM & Detection-Based Method \\
\hline DSBM & Data Source-Based Method \\
\hline $\mathbf{A B M}$ & Anomaly-Based Method \\
\hline MBM & Misuse-Based Method \\
\hline NBM & Network-Based Method \\
\hline HBM & Host-Based Method \\
\hline AI & Artificial Intelligence \\
\hline SL & Supervised Learning \\
\hline $\mathbf{U L}$ & Unsupervised Learning \\
\hline $\mathbf{A N N}$ & Artificial Neural Network \\
\hline Bi-RNN & Bi-directional Recurrent Neural Network \\
\hline CNN & Convolutional Neural Network \\
\hline DT & Decision Tree \\
\hline ID3 & Iterative Dichotomiser 3 \\
\hline DNN & Deep Neural Network \\
\hline KNN & K-Nearest Neighbor \\
\hline LR & Logistic Regression \\
\hline SOM & Self-Organizing Map \\
\hline SVM & Support Vector Machine \\
\hline RNN & Recurrent Neural Network \\
\hline RBM & Restricted Boltzmann Machine \\
\hline MLP & Multi-Layer Perceptron \\
\hline NB & Naive Bayes \\
\hline MDP & Markov Decision Process \\
\hline RF & Random Forest \\
\hline FSLC & Few Shots Learning Classifier \\
\hline FCM & Fuzzy C-Means \\
\hline BCI & Bienaymé-Chebyshev Inequality \\
\hline CART & Classification and Regression Trees \\
\hline REPTREE & Reduced Error Pruning Tree \\
\hline LSTM & Long Short-Term Memory \\
\hline Bi-LSTM & Bidirectional Long Short-Term Memory \\
\hline KM & K-Means \\
\hline GAN & Generative Adversarial Network \\
\hline GRU & Gated Recurrent Unit \\
\hline $\mathbf{A E}$ & AutoEncoder \\
\hline SAE & Stacked AutoEncoder \\
\hline VAE & Variational AutoEncoder \\
\hline DBN & Deep Belief Network \\
\hline HММ & Hidden Markov Model \\
\hline XGBoost & Extreme Gradient Boosting \\
\hline AdaBoost & Adaptive Boosting \\
\hline PCA & Principle Component Analysis \\
\hline LVQ & Learning Vector Quantization \\
\hline SD-IoT & Software Defined Internet of Things \\
\hline Src/Dst & Source/Destination \\
\hline
\end{tabular}

$\begin{array}{ll}\text { NMAP } & \text { Network Mapper } \\ \text { DoS } & \text { Denial of Service } \\ \text { DDoS } & \text { Distributed Denial of Service } \\ \text { U2R } & \text { User to Root } \\ \text { R2L } & \text { Remote to Local } \\ \text { XSS } & \text { Cross Site Scripting } \\ \text { DQN } & \text { Deep Q Network } \\ \text { PPO } & \text { Proximal Policy Optimization } \\ \text { TMANET } & \text { Tactical Ad hoc Mobile Network } \\ \text { TPR } & \text { True Positive Rate } \\ \text { FAR } & \text { False Alarm Rate } \\ \text { DDPG } & \text { Deep Deterministic Policy Gradient } \\ \text { DPI } & \text { Deep Packet Inspection } \\ \text { IoMT } & \text { Internet of Medical Things } \\ \text { DRL } & \text { Deep Reinforcement Learning }\end{array}$

\section{SOFTWARE-DEFINED NETWORKS (SDN)}

Due to the rapid changes of information structure from one place to another, governments and commercial organizations change their network configuration as per requirements of the fast-changing network paradigm on the internet. The intricate routine network creates an obstacle for many data centers to apply new services, connect various data centers with organizations. This is where SDN comes to aid. SDN architecture empowers the system to be centrally controlled and programmed using software applications rather than changing the structure of physical appliances [11]. The goal of SDN can be defined as improving network functions by allowing corporations as well as service providers to adapt rapidly to evolving market demands. The control plane is physically separated from the data plane, and it can intelligently control network resources. This intelligent network control is established when SDN decouples the network setup and traffic engineering from their underlying hardware infrastructure.

SDN separates network controller and data plane/switch activities with a protocol that updates forwarding tables in network switches. This enables on-the-fly network optimization and rapid response to changes in network traffic without the need for manual reconfiguration of current infrastructure or the purchase of new devices. SDN removes the control of network devices from the data they transmit and switches to software applications to regulate the behavior of the network residing outside of the networking devices (hardware) that offer physical connectivity. The logically centralized SDN controller which is a software entity that plays the central management role and does the job of flow management [17]. In a typical SDN outline, packet handling protocols are sent from a controller to the switch. An application program executing somewhere on a server and switches request supervision from the controller when required to provide it with traffic information that they manage. Controllers and switches typically interact through the OpenFlow [18] interface. There are three stages or layers in SDN architecture, which are shown in Fig. 2. 


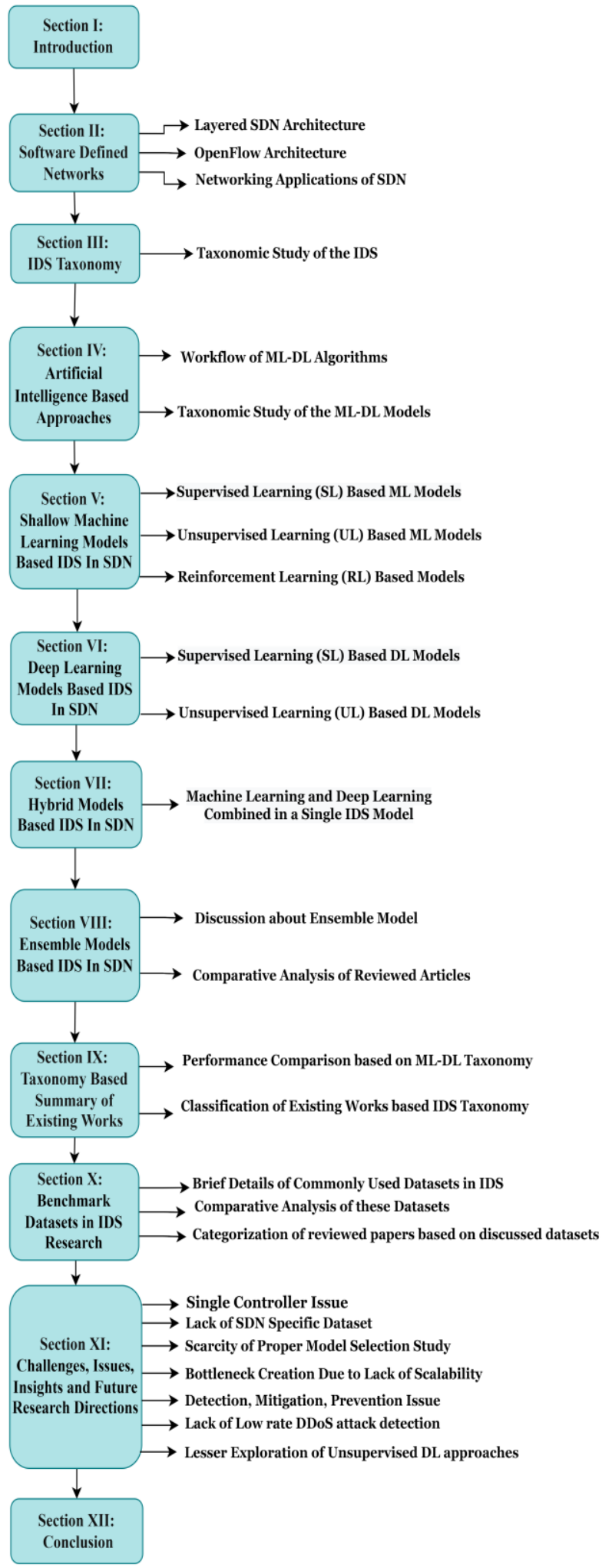

Fig. 1. Pictorial representation of the organization of this survey.

Data (Infrastructure) layer: It resides in the bottom layer of the SDN paradigm. It is comprised of physical switches, virtual or software-based switches routers and access point. softwarebased switches work on a variety of operating systems, including Linux. Some of the common virtual switches are Open vSwitch
[19], Pantou [20], Indigo [21], and Nettle [22]. Physical switches are hardware-based such as NetFPGA [23] and multiple vendor switches. Some of the NetFPGA-based implementations are [24], [25]. The functions data layer performs are dispatching, rejecting and transforming data according to the rules or policies provided by the control layer. The SDN data plane has a variety of devices that lack intelligence. They just simply carry out the controller's instructions or rules [26], [27].

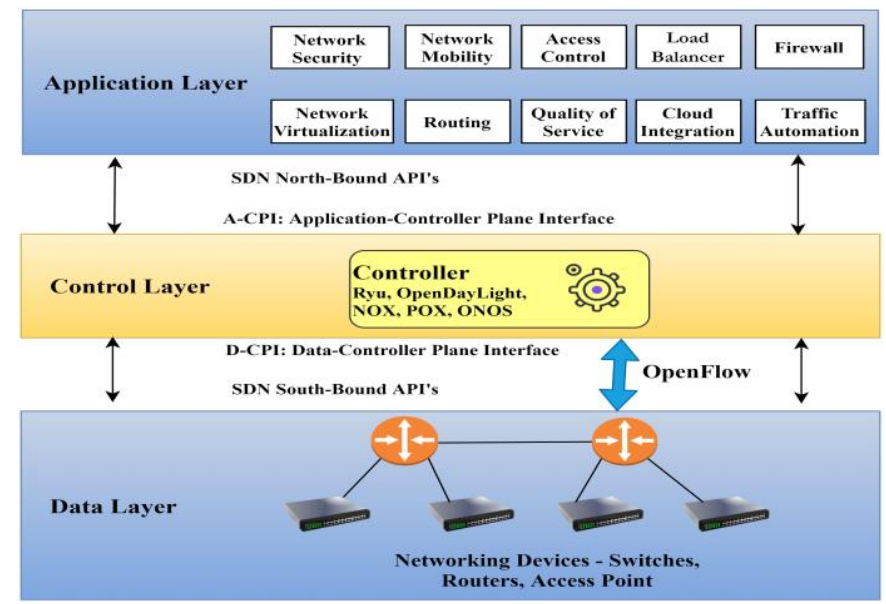

Fig. 2. Block diagram of a three-layered SDN architecture.

Control layer: Principal logical controller is the crucial unit of the control plane that facilitates overall SDN functionalities. The controller manages the entire traffic flow and is completely responsible for routing, sending, and dropping packets by programming [28]. Some of the common OpenFlow-based SDN controllers extensively used by the researchers are Ryu [29], POX [30] , NOX [31], OpenDayLight [32], Floodlight [33], and ONOS [34]. This layer controls the exchange of data between different applications and the dispatching devices.

Application layer: It is a pool of business applications. The application plane contains one or more modules, each managed solely by the SDN controllers over a resource collection [35]. Note that a typical IDS application is deployed in this layer. Though other studies have placed the IDS module in the control layer as well [36], [37]. Network security, mobility management, access control, load balancing, firewall implementation, quality of service, and cloud integration are some of the examples of applications that application layer deals, as shown in Fig. 2. The main function of this layer is to perform the required optimization for business network services. The following subsections cover the workflow and applications of SDN.

\section{A.Workflow of OpenFlow Based Switching}

OpenFlow (OF) is a standard managed by the Open Networking Foundation (ONF) [38]. OF is a protocol, which enables programmability of the data forwarding plane. The workflow of OF-based switching is governed by the Flow table, which stores a list of flow entries sorted by the priority of the 
flow, and a secure channel that connects the switch to the controller. The highest priority flow resides at the top of the table. An entry in the flow table consists of rules (matching fields), priority, counters, actions, timeouts, cookie and flags [38] as shown in Fig. 3. The flow rules are being applied to a corresponding flow [39], one entry per each flow. The rule (matching fields) consists of an algorithm that can be used for exact or wildcard matches with the information about the packet header that defines the flow. The information consists of ingress ports where OF packets are received, packet header fields such as IP/MAC address of source/destination (src/dst) and VLAN, switch port, etc., and metadata from the previous table. The priority field defines the matching priority of the flow entry to process the rules. When a packet is matched with the rules, the highest priority flow entry that matches the packet is selected and the counter is updated. When a switch finds a match according to the order of the priority flow, it applies the action, which defines the packet handling process (e.g., forward packets to ports/controller, drop, modification of source and destination IP/MAC address, etc.) to the matching packet. If it does not find a match, it performs the default operation of the packet, which is to transfer it to the controller. The maximum amount of time or inactive time before the switch expires a flow entry is defined as a timeout. Cookies are the flow entry identifiers specified by the $\mathrm{OF}$ controller. Flags change the way flow entries are managed by the controller. A brief workflow procedure of an $\mathrm{OF}$ switch scenario in SDN is presented in a stepwise manner in Fig. 4. Flow tables of an OF switch are numbered sequentially, starting from zero (0). The packet is first matched against flow entries in flow table 0 , which is the first flow table. If the flow entries do not match with the packet, then the packet is forwarded to the flow table with the larger number.

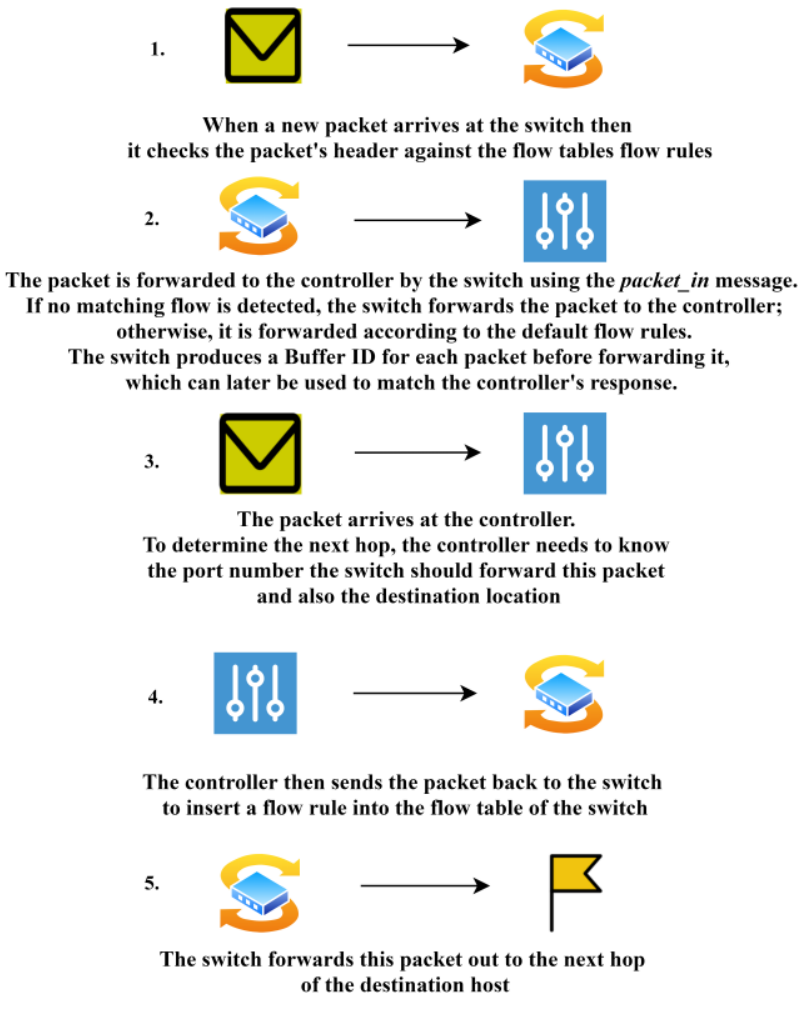

Fig. 4. Workflow procedure of OpenFlow-based switches.

\section{B. Networking Applications Based on SDN}

For a long time, the SDN research community has proposed and analyzed several network applications. These applications can be assembled into network management, inter-domain routing, traffic engineering, SDN security, virtualization of networks, and load balancing of application servers, and control of network access [18]. In the following subsections, some of them are briefly described.

\section{1) Traffic Engineering and Network Management}

Successful network management needs knowledge of the network's current state and prompt adjustment in the control area of the network. Authors in [40] proposed a multi-protocol level switching traffic engineering network, based on SDN Flow Tables Major Components

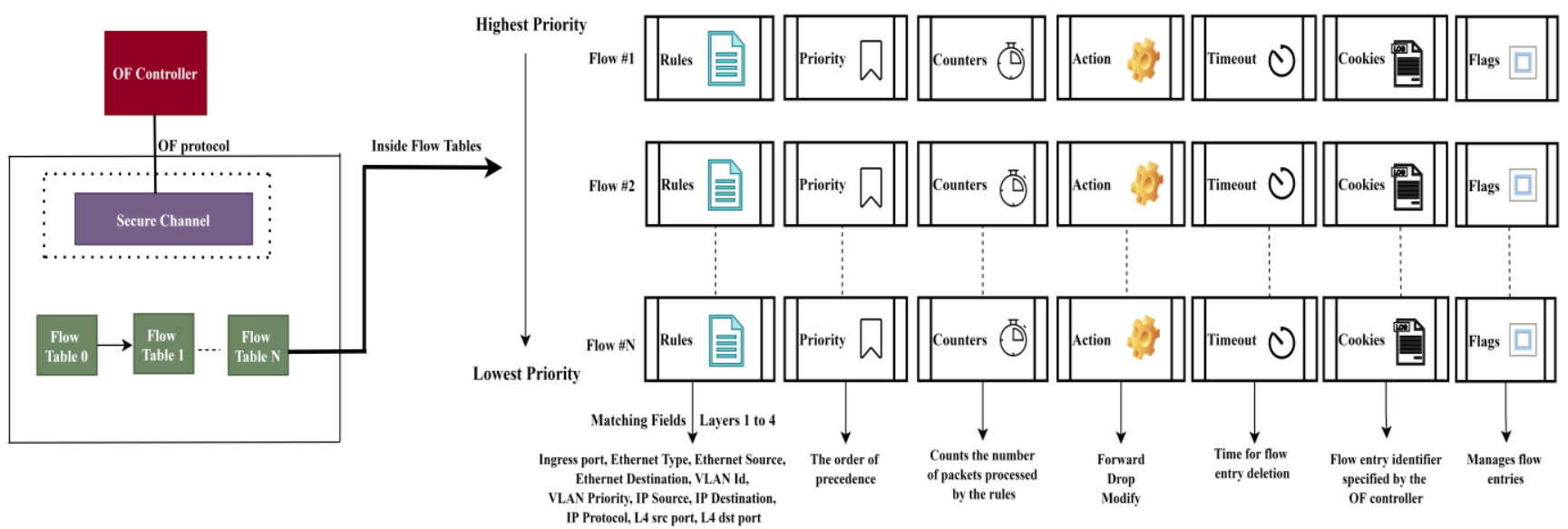

Fig. 3. Anatomy of the flow tables of OpenFlow-based switches. 
architecture that follows a new smart and complex paradigm of bandwidth allocation. The architecture handles the Quality of Service (QoS) and routing with QoS restrictions. They assessed the performance of their network by improving packet loads on HTTP, VoIP, video, and ICMP traffic. To precisely manage the network, the network's present status and the flow of information throughout the network needs to be known. In [41] the authors used the network data obtained by the principal controller to increase the capacity of the network and lessen packet failures. Specifically, they concentrate on the event where SDN is gradually integrated into a current network. Also, partial deployment of the optimization problem in the field of traffic engineering is formulated by the SDN controller. In [42] the authors discussed the user responsive interfaces of SDN based paradigm. The article presents an interface to the OF network to remotely manage the network, titled OMNI. It enables the administration of OF-based networks that is challenging due to a large number of configuration variables and various configuration management choices. OMNI watches and configures dynamic flow formation.

$\mathrm{Yu}$ et al. proposed OpenSketch [43], a traffic measurement architecture of SDN which is described by applications designed to support more personalized and complex measurements yet maintaining the precision of the SDN measurement by separating the data plane from the control plane. The authors suggest a 3 stage data plane framework consisting of counting, hashing and filtering that can be designed to accommodate a wide variety of measurement tasks such a traffic change identification, delay measurement, flow size dissemination assessment, etc. OpenSketch distinguishes the capacity of the data plane by associating many tasks of the control plane by constructing and allotting various computation tasks to accomplish this purpose.

\section{2) MiddleBox/Load Balancing}

To generate improved middlebox-based services such as load balancing, SDN solutions can be utilized. In [44], authors have used Multi-Path TCP (MPTCP) to allocate traffic around various routes in a WAN operated by OF. On one side, MPTCP can change the load balance with regards to the load on the path from flows of different traffic. Whereas, the OF software selects the best routes that can be used by diverse sub-flows of TCP and compels them to OF based switches. The authors in [45] proposed a method called MiceTrap, It controls the flow aggregation power offered by $\mathrm{OF}$ for managing the number of flowing mice and to implement a weighted routing algorithm that can accomplish better load balancing.

\section{3) Inter-Domain Routing}

Currently, the inter-domain routing method poses several serious issues, primarily due to its highly decentralized model. In [46], the authors suggest an inter-domain routing approach using the NOX [31] - OF infrastructure, initially developed for routing in business networks only. In [47], the authors proposed a framework made on top of Mininet [48] and Quagga routing suite [49] that uses the centralization functions of SDN to linearly lessen the border gateway protocol's convergence time as well as churn rates. Authors in [50] suggested a method for optimizing the route in an SDN-based setting. Route optimization was based on different constraints such as bandwidth, flow operations, and number of domains available while a mobile node travels through the SDN-based structure.

\section{4) Access Control and Network Security}

The invention of SDN provides a distinctive potential to commendably identify as well as cover security challenges in home-based and office-based networks. In [51], authors incorporate multiple traffic anomaly identification methods utilizing OF-based switches and NOX controllers to resolve system security concerns in the home-based and office networks. Studies showed that the SDN-based approach resulted in much more reliable in terms of various types of fraudulent activity recognition than the internet service provider driven method.

In [52], the authors suggest an OF based "Resonance" scheme that offers complex access management policies to enforce network access control in network components. It helps switches to take action, such as dropping of packets, to implement increased security policies. Identification and protection against DoS and DDoS attacks have been studied many times in the field of SDN. In [53], the authors detected DDoS attacks by calculating the occurrence of the flow of traffic. With the occurrence of the stream exceeding the stated level, it is presumed that there is a DoS attack. In such instances, the SDN controller suggests the switches to drop those packets which are part of the spiteful stream to minimize the impact of the attack.

\section{5) Network Virtualization (NV) Based on SDN}

In certain ways, NV and SDN are strongly interlinked [54]. However, due to many connected elements in a network and their complex communication structure, NV and SDN integration is a challenging task. In [55] authors presented an software defined network virtualization framework that combines SDN and NV. The SDNV model blends the SDN concept of data isolation and control plane with the NV principle of convergence operation functions from services and infrastructure, thereby offering a simple broader picture of SDN and NV integration.

In [56], the authors suggest a hierarchical policy-based architecture named PANE that produces flow-specific inputs from virtualized specifications, which reveals an API via a northbound interface which could be utilized to query a virtual network. The framework is capable of identifying conflicts whenever there is a requirement for a new virtual network. 


\section{IDS in SDN - Concept}

The goal of the IDS is to inspect traffic from the network, monitor irregular traffic regarding unusual behavior or trends, and notify the network administrator. The ML-DL-based IDS implementation in SDN can be divided into three key steps: attack identification, reporting, and mitigation [57]. In the attack identification step, the IDS module collects data, and using the ML-DL-based model; it detects the attack in SDNs. The reporting step occurs when the IDS module sends an alert to the controller regarding the detected attacks, as shown in Fig. 5. In the mitigation step based on the received alert from the IDS module, the controller can change the flow rules for incoming packets and transfer those rules to the OF-based switches to block the corrupted network traffic. Within that OF-based switches, the changed flow rules are saved indefinitely. An ideal IDS must-have features such as higher precision, improved performance, low degree of fault tolerance, fast, low memory consumption, and robustness [58]. In the next section, we provide a taxonomic study of different domains of IDS.
An anomaly is simply a suspicious event from the standpoint of security [63]. The crucial point of ABM is the settings of a standard behavior profile. Any behavior, which deviates beyond the standard profile, can be treated as uncommon behavior. The anomaly detection has robust generalized nature and can identify unidentified attacks. Drawbacks are excessive wrong alert levels and incapability in offering probable causes for an irregularity in the system [6].

MBM is also referred to as signature-based detection method. The primary intuition of MBM is to characterize attack procedures as signatures. It saves each attack procedure in its database and checks each new signature sample with the previously stored signatures to detect misuse. One of the benefits of this system is that it has a low incorrect warning rate and defines the attack natures with the cause in detail. One of the drawbacks is that it needs a large database to store all the signatures; also, this system lacks in identifying unfamiliar attack [63]. This strategy works well in general, except when we encounter a novel attack or one that has been purposefully

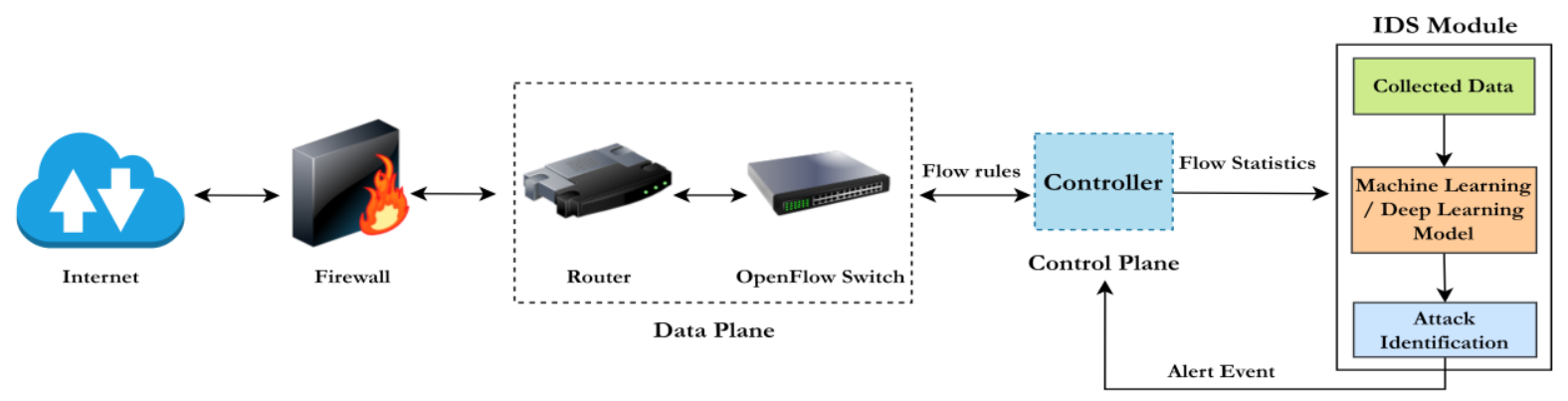

Fig. 5. A high-level view of the deployment of IDS in SDN architecture.

\section{IDS TAXONOMY}

Whenever someone attempts to get unauthorized information to harm the system, it is defined as an intrusion. We need an effective system to understand those abnormal patterns in data traffic and keep the system secure. The key task of IDS is to monitor suspicious behavior that goes beyond the normal procedures of action of every individual host in the network, creates warnings when such action is detected, and respond to that chary behavior. A taxonomy of the IDS is presented in Fig. 6.

Mainly IDS classification approaches are categorized into two parts, namely Detection-Based Methods (DBM), and Data Source-Based Methods (DSBM) [6], [59]-[61]. This survey includes these two types of IDS classification techniques in SDN paradigm, with the DSBM serving as the primary classification criteria and the DBM serving as a secondary classification criterion. Regarding DSBM and DBM, the survey focusses on ML and DL-based methods in IDS solution. DBM is further categorized into two classes: Anomaly-Based Method (ABM) and Misuse-Based Method (MBM). On the other hand, DSBM is further categorized into two more classes: Network-based and Host-based [62]. designed to not match previous attack signatures. Large number of studies adopted the MBM-based IDS solution for SDN, such as pattern matching, expert system-based MBM-based IDS solution [64]-[67]. Pattern matching technique functions by assuming that the infected hosts show analogous behavioral patterns which are unlike from benign hosts [68]. Several studies [69], [70] have adopted pattern matching technique for developing SDN-IDS solution. To design a misuse detection system in SDN-enabled infrastructure, the expert system is utilized for decision-making based upon behavior-profile [71], [72]. A comparison between $\mathrm{ABM}$ and $\mathrm{MBM}$ is presented in Table III.

We can further classify IDS based on data gathered from different input sources to classify normal and abnormal attacks. Network-Based Method (NBM) and Host-Based Method (HBM). NBM-based IDSs are used to analyze the arrival and departure of traffic to and from nodes in the network and raises alerts if an intrusion is detected [73]. It tracks the extracted network traffic like packet captured, NetFlow, and further data traffic streams [8]. NBM-based IDSs are capable of detecting particular kinds of protocol oriented and network attacks [74]. ML approaches have been widely used in the field of NBMbased IDS, particularly in the task of classifying network traffic 
[75]. Researchers have explored a great deal in the possibility of robust NBM-based IDS development using ML, and DL approaches in the last decade [63], [76]-[81]. On the downside, NBM-based IDS would only track that traffic going through a particular network sector. Most NBM-IDSs do not process encrypted packets. As a result, the encrypted packet might allow a malicious and unnoticed network breach until more serious network intrusions occur. Because of the nature of the NMB-IDS solutions and their requirement to analyze protocols as they are collected, NBM-IDS solutions can be subject to the same protocol-based attacks that network hosts are vulnerable to [1].

The HBM is an IDS solution that monitors the computer infrastructure on which it is deployed, monitoring traffic and logging unwanted and malicious activities. The HBM will defend local machines and serve as a final line of defense, whereas the NBM will maintain the network backbone safe and secure [82]. HBM-based IDSs detects the non-official operation by matching the new version of the available archives and logs with the earlier version [83]-[86]. HBM-based IDSs monitor log files created by running programs, generating a historical record of events and functions that can be easily searched for abnormalities and evidence of an intrusion. It also compiles the $\log$ files and allows a user to organize them in ways that correspond to the directory structure of the designated log file server, allowing a user to search or sort the files in various ways. Numerous studies [82], [85], [87]-[92] have adopted ML and DL-based algorithms such as SOM, HMM, MDP, KNN, CNN, RNN, and LSTM to effectively design host-based IDSs. The drawbacks of HBM-based IDSs are that it consumes host assets, are contingent on host stability, and therefore incapable to recognize attacks effectively. A host-based IDS cannot provide the complete solution for the network security all by itself. Though monitoring the host seems practical and obvious, there are three major drawbacks: The visibility is confined to a single specific host; the IDS process consumes many resources like memory, file system organization, CPU, storage, implementation of commands and procedures etc., potentially affecting host performance; and lastly, attacks are not detected until after they have already reached the host [93]. The primary downside is that it must be deployed on all hosts requiring protection from intrusion, which provides additional processing overhead to each node and eventually reduces the IDS's performance. A comparison between NBM and HBM is presented in Table IV. A solid business network should ideally include both hostbased and network-based IDSs.

\begin{tabular}{|c|c|c|}
\hline \multicolumn{3}{|c|}{ TABLE III } \\
\hline CoMPARISON BETWEEN ABM AND MBM \\
\hline Domain [94] & $\begin{array}{c}\text { Machine Learning Model, } \\
\text { Time Series Based } \\
\text { Model, Statistical Model. }\end{array}$ & $\begin{array}{c}\text { Expert System, Pattern } \\
\text { Matching, Finite State } \\
\text { Machine. }\end{array}$ \\
\hline $\begin{array}{c}\text { The efficiency of } \\
\text { Detection [95] }\end{array}$ & $\begin{array}{c}\text { Depends on a model } \\
\text { hurdle. }\end{array}$ & $\begin{array}{c}\text { High, detects attack } \\
\text { with a lower degree of } \\
\text { false alarms. }\end{array}$ \\
\hline New Attack [8] & $\begin{array}{c}\text { Can detect both identified } \\
\text { and new attacks. }\end{array}$ & $\begin{array}{c}\text { Unable to identify new } \\
\text { attack types which it has } \\
\text { not seen before. }\end{array}$ \\
\hline $\begin{array}{c}\text { Performance } \\
\text { Metrics [96] }\end{array}$ & $\begin{array}{c}\text { A large number of false } \\
\text { alm rate, lesser number }\end{array}$ & $\begin{array}{c}\text { A large number of } \\
\text { missed alarm rate, lesser } \\
\text { number of false alarm } \\
\text { rate. }\end{array}$ \\
\hline Scalability [97] & High & Low \\
\hline Speed [10] & Low & High \\
\hline
\end{tabular}

Audit logs and system calls are the two primary data sources that may be used for the HBM-Based IDS solution. Audit logs are a collection of events generated by the Operating System (OS) to execute specific tasks and operations. In contrast, system calls describe the behavior of each user-critical application executing on the OS. Auditing is a technique that a host uses to identify and record security-related activities. The records of such activities are maintained in audit log files, which typically contain records of all the operating processes, the consumed internal memory, and the file systems that these processes use for their execution [98]. The core notion behind using system calls to detect an intrusion is that an abnormality in the running application will also influence how it interacts with the OS.

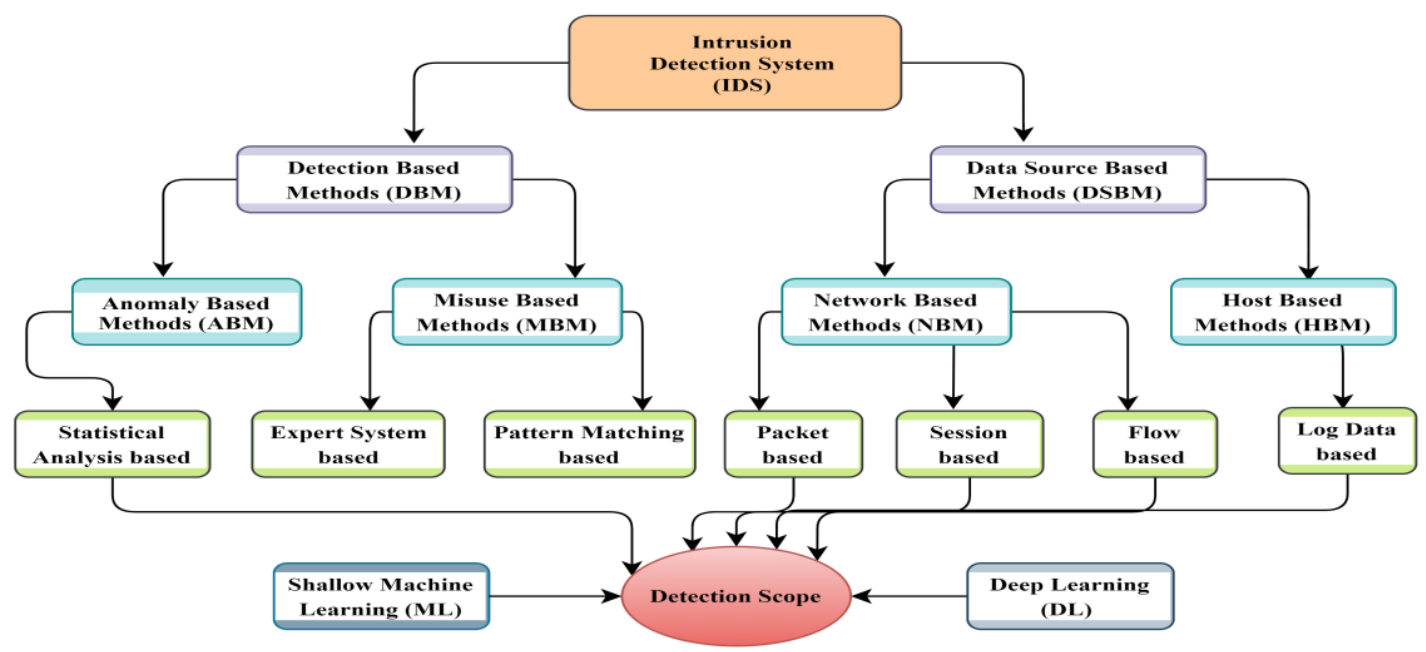

Fig. 6. Illustration of an IDS taxonomy. 
Log-based IDS uses a host's logs after logging into a machine as the primary information to detect attacks on a particular environment [99]. This approach's benefits are that it does not require costly hardware, system activity visibility, etc. Logbased IDS typically contains the following components: log file/data collection, pre-decoding, decoding, analysis, and report the consequence of the events [100].

\begin{tabular}{|c|c|c|}
\hline \multicolumn{3}{|c|}{$\begin{array}{c}\text { TABLE IV } \\
\text { COMPARISON BETWEEN NBM AND HBM }\end{array}$} \\
\hline Criteria & NBM & HBM \\
\hline Data Source & Network Traffic. & $\begin{array}{c}\text { Audit Trails, Log Files, } \\
\text { System Calls. }\end{array}$ \\
\hline Domain & $\begin{array}{c}\text { Packet Parsing, Payload } \\
\text { Analysis, Traffic } \\
\text { Grouping, Feature } \\
\text { Engineering, Sequence } \\
\text { Feature, Statistical Feature } \\
\text { Analysis. }\end{array}$ & $\begin{array}{l}\text { Text Analysis, Feature } \\
\text { Engineering. }\end{array}$ \\
\hline $\begin{array}{l}\text { The efficiency } \\
\text { of Detection }\end{array}$ & High & Low \\
\hline Drawbacks & $\begin{array}{l}\text { Hard to assess a network } \\
\text { with high measurement. }\end{array}$ & $\begin{array}{l}\text { Host-Specific. Needs to } \\
\text { be installed on every } \\
\text { host in the system. }\end{array}$ \\
\hline
\end{tabular}

In session-based IDS, the network traffic for a specific session is analyzed to detect anomalous traffic. The session-based IDSs, in particular, extract a few fundamental features from the header field of the incoming packets and pick payloads of the network application layer within a specific session as features. A record is made up of the header features and payloads of a session. The records are then put into a supervised or unsupervised ML-DLbased algorithm, which extracts significant features for categorizing legitimate and malicious network traffic [101] [103].

Statistical approaches use a statistical function of network traffic parameters to create a profile of regular network traffic. This regular traffic profile is used to check for unnoticed inbound traffic. Statistical metrics are used to calculate the similarity of incoming network traffic with the profile of regular network traffic. If the similarity measure exceeds a predetermined threshold, the flow is classified as malicious or benign [1], [104], [105]. The raw captured packets are evaluated for a series of time in statistical analysis-based IDS [106] and the packets' variances for behavior profile analysis in the packet's header. Univariate, multivariate, Markov decision model, and time-series analysis are some of the standard statistical approaches in flow-based IDS solution development [104], [107]-[111].

Packet-based analysis examines the whole packet (payload + header) for application-layer data looking for specific rules or signatures [112], [113]. In high-volume networks, packet-based inspection necessitates a large amount of memory and CPU time. This is due to the data's low abstraction level, the high frequency of data arrival, and the possibility of significant quantities of data per instance of information [114].
Flow-based IDSs examine just the header of the packet and do not examine the internal payload [104]. Flow-based data is the aggregated information of packets related to the network traffic in a flow, thus the amount of data to be examined is reduced. A record in a flow generally includes the IP addresses of the hosts, network ports, protocol, flow priority, several counters, quantity of data, and the time of the flow occurred [115]. There is hardly any article found which used expert system and pattern matching based analysis using ML-DL techniques regarding deployment of IDS solution for SDN paradigm. Besides, these are subdomains of misuse-based method (MBM), which is not part of this survey. This survey focuses on NBM, HBM, and ABMbased IDSs adopting ML-DL approaches. Therefore, these two types are out of the scope of this survey.

\section{ARTIFiCial InTELligenCE BASED APPROACHES}

In this section we provide an ML-DL based taxonomy to review the existing literatures that used to formulate IDS solution for SDN environment. Artificial Intelligence (AI) is an extensive term for a group of systems that allow machines to emulate intelligent human expertise. AI uses approaches such as ML, DL, and RL to solve real-world problems. The researchers investigated the use of shallow ML and DL approaches to meet the criteria of efficient and functional IDS. Both shallow ML and DL fall under the broad umbrella of AI and seek to learn meaningful information from big data. Though DL is a subset of ML, for this survey, we are reviewing each paper based on the traditional/shallow ML and DL-based method/technique adopted to develop IDS solution in SDN. Hence, ML and DL are set up at the same rank in our taxonomy presented in Fig. 7. The way each algorithm learns is where ML and DL differ. In the last decade, there has been an upsurge where the deployment of MLDL methods in IDS is yielding better performance than traditional IDS systems in fields like SDN, mobile cloud, traffic engineering, and service optimization.

ML-DL methods learn from the knowledge it gathers from the data during the training of the model. In general, for IDS, there exist two types of ML-DL algorithms: supervised ML-DL algorithms and unsupervised ML-DL algorithms. An IDS model attempts to differentiate between regular and malicious traffic patterns in the supervised ML method, provided a set of network flows with labels (normal/malicious) before training [116]. The manual labeling of each data is costly as well as time intensive. However, unsupervised ML algorithms gather vital information or features directly from the unlabeled dataset. Supervised MLDL methods show much superiority than unsupervised ML-DL methods in IDS efficiency as observed in the works of previous researchers.

IDS has been using mainstream ML algorithms such as LR, SVM, DT, NB, RF, CART, ID3, KNN, SOM, K-Means and many more for a long time. Often it is observed that a collection of ML models-supervised and unsupervised is used in 
ensemble to perform a task. An ensemble-learning algorithm may combine one or more different types of algorithms shown in the taxonomy presented in Fig. 7. Major problems within this methodology are the need of availability of data and retraining of the data. Often, they are prone to overfitting and fail to detect unknown attacks.

In the following, we briefly discuss the workflow of ML-DL algorithms.

\section{Workflow of ML-DL Algorithms}

Building a system through ML-DL algorithms generally pass through several steps before achieving the outcome. These steps are data collection, data processing, model selection, training, evaluation, parameter tuning, and testing [117]. However, sometimes these steps could be varied subject to the particular task and available data and features. A workflow of a typical MLDL model is presented in Fig. 8.

Data Processing: Collected raw data is generally noisy in nature. It may require some pre-processing tasks like removing duplicate data, identifying anomalies, discarding erroneous data, scaling, normalization, feature engineering, transformations, etc. Besides, the format of the data may require to be converted for some specific ML models. Data processing also enables data visualization to identify biases.

Model Selection: A specific ML-DL method is selected in this step. Results of different ML models can be varied significantly. That is why the model should be chosen carefully.

Training: In the training step, the selected ML model is being learned by the collected data. The aim of this training step is to prepare the model to perform better against unseen data.
Evaluation: For the performance measurement of a selected model, various evaluation metrics and sampling techniques are used in this step. The trained model is often tested against unseen data using separate validation set or $\mathrm{k}$-fold cross validation. Use of unseen data depicts the possible performance measure of the model in the practical scenario.

Tuning: Tuning is the process of enhancing a model's performance while avoiding overfitting or excessive variance. In ML, this can be achieved by choosing optimal hyperparameters. The performance of the ML models depends on choosing the appropriate value of the different hyperparameters. This is often achieved by hyperparameters searching techniques like random search, grid search, neural architecture search, etc.

In the next section we discuss those studies which have used shallow ML-based algorithms to develop IDS solutions specific to SDN.

\section{Shallow Machine Learning (ML) Models BASED IDS IN SDN}

ML models can be trained based on different learning approaches. They can be categorized as Supervised Learning (SL), Unsupervised Learning (UL), and Reinforcement Learning (RL). SL-based models are trained with labeled data, as shown in Fig. 9. In IDS, most of the intrusion or anomaly detecting tasks are classified using different SL-based models. On the other hand, UL-based models gather information from unlabeled data, as shown in Fig. 10. In contrast, RL-based models rely on continuous feedback from critics based on some particular actions. However, in ML-based IDS, RL-integrated models are relatively rare compared to the other two approaches. An MLDL taxonomy is showed in Fig. 7. The taxonomy reflects the models which are used commonly in IDS.

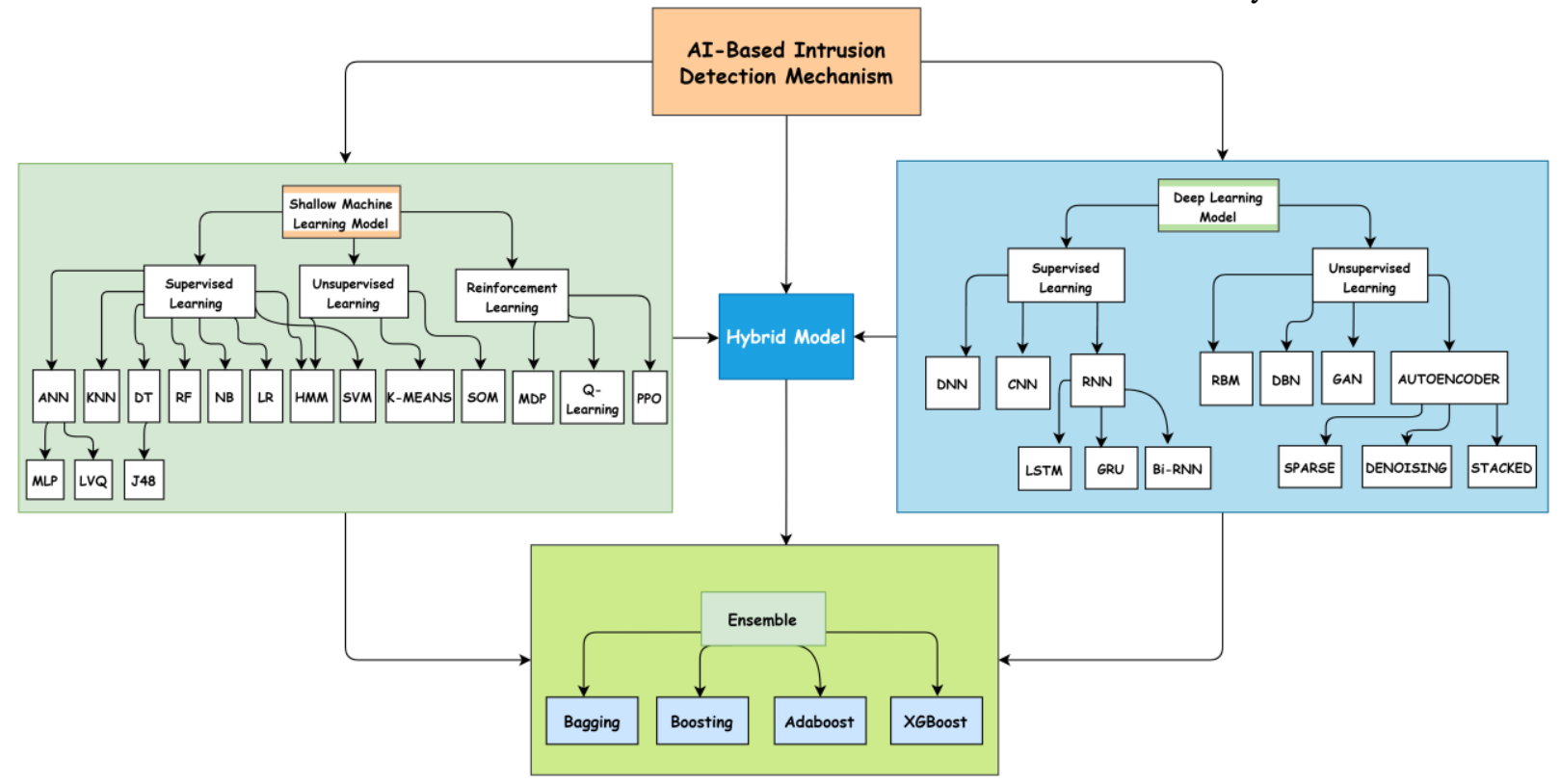

Fig 7. Taxonomy of ML-DL algorithms utilized in IDS. 


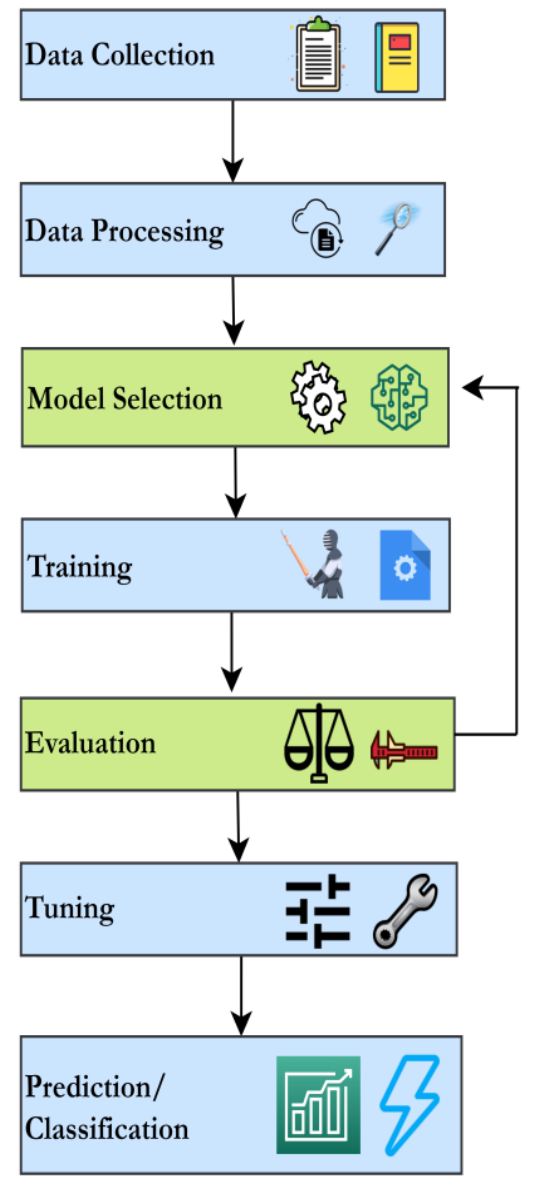

Fig. 8. Workflow of ML-DL based algorithms.

\section{A. Supervised Learning (SL) Based ML Models}

In the existing IDS literature in SDN, most of the researchers performed classification of network traffic using some supervised ML models. In SL, the machine is trained with welllabeled dataset and aids in the prediction of unseen data as shown in Fig. 9. It implies that some input data has already been marked with the accurate output label. DT, NB, RF, KNN and SVM are used more frequently in intrusion detection compared to other supervised models.

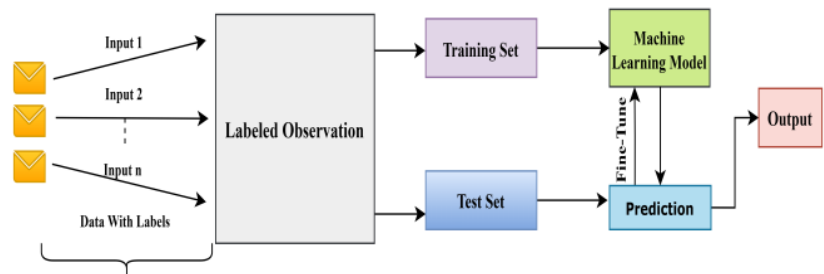

Fig. 9. Supervised Learning (SL) method structure.

\section{Decision Tree (DT)}

The DT is a tree-based representation of data and has a structure of nodes. Each node represents a decision to be taken based on features, and the leaf nodes of the tree denote the class label [118]. The route from the root node to the leaf node of a particular class depicts the classification rule.

\section{Naïve Bayes (NB)}

NB classifier is a probabilistic ML model. NB classifier assigns some probability measure by calculating the frequency or density of the feature values provided by the input dataset. NB classifier assumes that every feature is conditionally independent given the label [119]. For any labeled data, Eq. 1 can be applied, where $\mathrm{C}$ represents the class label and $\mathrm{F}_{\mathrm{i}}$ denotes different attributes or features.

$$
\mathrm{P}\left(\mathrm{C} \mid \mathrm{F}_{1} \ldots \ldots \ldots \mathrm{F}_{\mathrm{n}}\right)=\frac{\mathrm{P}(\mathrm{C}) * \mathrm{P}\left(\mathrm{F}_{1} \ldots \ldots \ldots \mathrm{F}_{\mathrm{n}} \mid \mathrm{C}\right)}{\mathrm{P}\left(\mathrm{F}_{1} \ldots \ldots \ldots \mathrm{F}_{\mathrm{n}}\right)}
$$

\section{Support Vector Machine (SVM)}

SVM finds a hyperplane to separate the data into two different classes [120]. SVM finds some points nearest to the hyperplane separating the classes; these points are called support vectors. The goal is to maximize the margin or width of the hyperplane, separating the support vectors selected from both classes. For a binary classification regarding intrusion or regular traffic, the decisive SVM function could be expressed through Eq. 2.

$\mathrm{F}(\mathrm{x})=\mathrm{w}^{\mathrm{T}} \cdot \mathrm{x}+\mathrm{b}$

Here, $w=\sum_{i} \alpha_{i} y_{i} x_{i}, \alpha_{i}=0$ for all cases except for the support vectors lying precisely at the splitting hyperplane, and $y_{\mathrm{i}} \in\{1,-1\}$ refers to the labels, and $\mathrm{x}_{\mathrm{i}}$ represents data points.

\section{K-Nearest Neighbor (KNN)}

$\mathrm{KNN}$ is an instance-based lazy classifier that performs relatively well without any assumptions about the underlying data [47]. This nonparametric feature is a compelling aspect, as most real-world data do not reflect any fundamental foundations and assumptions, e.g., linear independence, uniform or normal distribution, etc. It is widely used in the IDS. With a smaller k, there is a higher chance of overfitting, with the model being a bit complex. On the other hand, with a larger k, the model's fitting capability gets weaker, but the model becomes simpler. The Euclidean, Manhattan and Minkowski distance functions of KNN for calculating distance between two points $\left(\mathrm{P}_{\mathrm{i}}, \mathrm{Q}_{\mathrm{i}}\right)$ in the dataset are shown in Eq. 3, 4 and 5.

$$
\begin{aligned}
& E(P, Q)=\sqrt{\sum_{i=1}^{k}\left(P_{i}-Q_{i}\right)^{2}} \\
& M(P, Q)=\sum_{i=1}^{k}\left|P_{i}-Q_{i}\right| \\
& M K(P, Q)=\left(\sum_{i=1}^{k}\left(\mid P_{i}-Q_{i}\right)^{v}\right)^{\frac{1}{v}}
\end{aligned}
$$

Here, $v=\{1,2\}$ 


\section{Supervised ML Based IDS in SDN}

Intrusion detection in SDN using ML techniques has gained popularity because of the exceptional upsurge of vast amount of success of ML algorithms in network security. In the following, we review some of the supervised ML-based IDS solution in SDN paradigm.

Intricate attacks may inject malicious payloads into the packets. By inspecting packets deeply, Cheng et al. [121] propose an OpenFlow-based deep packet inspection method on the SDN environment that incorporates the DT, RF, Multinomial-NB, KNN, SVM and RF ML algorithms. First, the method introduces a primary recognition mechanism linked with the filtering of IP at the flow level. After that, the packets are checked by a deep packet inspection (DPI) engine, which comprises two key components: unencrypted traffic inspection and encrypted traffic inspection. For the unencrypted traffic, the proposed system extracts the payload (tri-gram frequency based on TF-IDF, linguistic) features. For encrypted traffic, the system extracts some prominent features such as TLS cipher suites to train the classifier to classify malicious encrypted packets. This work proposes a customizable packet-driven sampling method based on the linear prediction to overcome the resourceperformance issue at the packet-level granularity.

Yu et al. [122] also performed DPI-based traffic classification on the SDN environment. For the classification task, they have used the Tri-Training mechanism [123] consisting of SVM, $\mathrm{KNN}$, and NB classifier after collecting flow features from the network traffic. Several other studies also performed DPI mechanisms to detect attacks in the SDN paradigm. [113], [124] [126]. In ref. [124] Lin et al. add a two-tier mechanism to classify traffic flow to the traditional SDN architecture. First, rather than the controller, it conducts traffic classification on the data plane. If the initial categorization module cannot handle the traffic, it will be forwarded to a DPI module. However, forwarding traffic to specific DPI engines typically results in global network load and link usage problems. However, since malware producers switch their product distribution from HTTP to HTTPS to avoid payload analysis, DPI cannot be relied on to extract characteristics for malware identification. For this reason, Cusack et al. [127] proposed a scheme that leverages programmable forwarding engines (PFE). The flow records created by PFEs offer per-packet information and allows to extract flow characteristics for ransomware classification. The architecture is divided into two sections: processing of stream and classification. The stream processor reads a PCAP, executes, maintains a customized flow table, and collects flow features for the ML-based classifier. The classifier uses the retrieved features to train a model that can detect ransomware.

In another study, Cabaj et al. [126] utilized the SDN method for ransomware detection by tracking the packet lengths of HTTP POST messages using DPI. Once the ransomware was detected, the IP addresses of the command-and-control servers were located and excluded.

Song et al. [128] propose a risk awareness-based IDS in SDN. The proposed model has four steps: i. Data processing, ii. Data modeling, iii. Decision-making, and iv. Response scheme. Data processing steps are designed to find appropriate features set. Two supervised ML algorithms (DT and RF) have been used in the data-modeling step to predict malicious activities. Decision making step analyzes the result from the data-modeling step and directs a signal to the response scheme to initiate a security response. In the response step, the system changes the flow rules for different network routes. Altering the traffic flow of malicious hosts will guard the system from overwhelming potential traffic. Thus, the system could maintain a balanced flow among all the routes.

Silva et al. [129] develop ATLANTIC project to detect abnormal traffic streams and classify them using SVM. It is twophase architecture to detect the anomaly and mitigate the traffic to achieve normal flow. In the first phase, tables are maintained to record the traffic flow. Further, the table records are utilized to find deviation using different entropy theory. In the second phase, SVM is used to categorize the abnormal traffic flow.

Nanda et al. [84] propose a system that can identify malicious connections using four ML algorithms, namely NB, DT, BayesNet, and decision table. The prediction results were utilized by SDN to implement security measures to protect vulnerable hosts. For their experiment, they used log data from different architectures and classified them into two other classes.

Peng et al. [130] present an SDN-based IDS that detects an anomaly in the flow of the network. In their system, the controllers' flow collection unit gathers information from the flow table of the OF switches, collects the flow data features, preprocesses the features, and implements the detection of anomaly flow from the normal flow using the KNN algorithm.

Satheesh et al. [131] present a priority-based machine learning approach using SDN to manage data packet flow across the network. Their model obtains network information and uses the principle of packet classification information and adjusts flow rules to block malicious information flows.

Abubakar and Pranggono [132] propose a neural networkbased IDS solution for SDN. They first built a simulated testbed, which simulates the actual scenario by offering a mechanism for signature-based attacks. The model is then configured to provide anomaly-based detection and incorporated the later model into the signature-based model to detect a previously unseen attack undetected by the first method. They achieved a $97.3 \%$ detection rate using pattern recognition technique.

Schueller et al. [133] present a two tier hierarchical IDS for SDN by combining the flow based properties and packet based properties of the network data. They have developed the flowbased IDS using SVM algorithm with DARPA dataset. It matches the flows of SDN traffic against the database that 
comprises flow rules. For advance assessment of packets, irregular flows are then transferred to the packet-based IDS.

Ajaeiya et al. [134] implement a model to determine potential attacks such as DoS and brute force attack variants such as HTTP credential and SSH. The major benefit of SDN is that OF switches sends statistical information per flow item to the controller. The proposed approach uses the OF switches' statistical features to extract flow patterns, features and combine them before labeling for classification model.

Wang et al. [135] presented a flow control-based IDS. Their enhanced behavior-based SVM model detects and monitors traffic for abnormal flow. They have used the Support Vector Classifier to select the most qualified features from a set of raw features. By doing this, they have enhanced their detection accuracy. For feature reduction, they used the ID3 DT algorithm.

Using SVM for the DDoS attack detection, Yang and Zhao [136] propose a 3-module based IDS model. The first module collects traffic data where a RYU controller extract features through statistical flow analysis information, then identifies DDoS attacks on the SDN campus network in the second module, and finally adjusts the flow delivery rules in the last module to withstand the DDoS attack.

By analyzing the current IDS solution's limitation in the SDN paradigm, Alshamrani et al. [137] present an ML-based DDoS attack recognition and mitigation scheme. They have studied misbehavior attack, where an attacker can fool the controller by sending a valid packet as the first packet of a flow, then sends malicious packets later, and NewFlow attack where packets with new and unseen flows are sent to the controller creating a bottleneck. NSL-KDD dataset was chosen as the dataset and selected subset of features form that dataset using ranker, greedy and genetic algorithm for the model training combining with correlation-based feature selector that ranks the subsets of feature following a correlation heuristic evaluation function [138].

Vetriselvi et al. [139] propose a ML based two level IDS solution for SDN paradigm. They created the IDS by combining ML and genetic algorithms. Their model is separated into two levels, the $1^{\text {st }}$ level (deployed in the switch) is used to detect the attacks and the second level categorizes the attack types.

Leveraging the metaheuristic optimization BAT algorithm [140] and ML based RF algorithm, Li et al. [141] project an artificial intelligence based 2-stage IDS solution for SoftwareDefined IoT (SD-IoT) networks. They have selected five class flow features from KDD CUP 99 dataset using a modified BAT algorithm with differential mutation and swarm division process. A modified RF algorithm and weighted voting mechanism were then used to adapt the weights of the sample data to categorize the flow from the initial stage. Their model achieves lower false alarm rates due to optimal and core feature selection processes regarding attacks in the SD-IoT networks.

Akbas et al. [142] studied ML algorithms' usage and effectiveness in the SDN paradigm to detect intrusion. To detect
DDoS attacks in SDN, their IDS solution was integrated along with the POX controller. They have used KNN, DT, and SVM for the IDS. Detection performance was evaluated using KNN, DT, and SVM algorithms using the NSL-KDD dataset. Using feature selection, they have selected only six features from the forty-one available features in the dataset.

Boero et al. [112] investigate an SVM-based IDS solution in the SDN for detecting various malware intrusions in the network. Using the Entropy-based Information Gain (IG) method, they have selected core features based on probability density estimation, contributing more to the intrusion. By ranking the features, they have selected nine major features well suited to SDN architecture to carry out the IDS solution in this study.

Elsayed et al. [143] have executed a careful investigation of the common ML methods for identifying attacks in the SDN. A study about the linearity and non-linearity issues of the datasets was carried out in their study. The t-Distributed Stochastic Neighbor embedding was used to cluster the dataset's malicious and normal traffic as a nondeterministic measure. Principal Component Analysis (PCA) algorithm was then used to reduce the dataset's dimensionality and thus reduced features from 122 to 20. They have suggested that traditional ML algorithms fail to classify a wide range of SDN attacks with precision; hence, the DL-based approach was suggested to work with complex feature analysis for the detection task.

An ML-based TCP-SYN and ICMP Flood attack mitigation method was proposed by N.N. Tuan et al. [144] in the SDNenabled Internet Service Provider (ISP) networks. For the training dataset, they have used CAIDA 2007 and also generated traffic by using the DDoS traffic generator testbed "Bonesi" [145] for additional training of the model. The attacker exploits the Network Address Translation mechanism of the ISP. When the controller (POX) [30] obtains a "packet_in" message for any unknown flow of packets, the KNN-based IDS module identifies the packets as normal or traffic, based on ICMP packets volume and by calculating the port's entropy during TCP traffic. For the mitigation part, they have used the time monitoring concept to calculate the port's entropy, based on the number of source IP address and their corresponding ports that are open. Two XGBoost events were used in the study, one is for the prediction of monitoring windows, and the other is for predicting the threshold value of the monitoring window.

Afsaneh et al. [146] propose a model for DDoS attack classification method combining three phases: the collector section, the entropy-based section, and the collection section. The system aims to train the classification model with recent data to overcome relatively older data dependency. They applied three different datasets (UNB-ISCX, CTU-13, and ISOT) to test the classification model's performance, where the chosen models are J48, BayesNet, Random Tree, REPTree, NB, and LR.

Pérez-Díaz et al. [147] propose a modular architecture for low rate DDoS attacks such as DDoSSim, hulk, Slowloris, etc. 
detection and prevention in SDN. The first module, the IPS running on top of the ONOS SDN controller, captures the flow and sends it to the IDS. In the second module, the ML-based IDS detects the flows and sends back the detection result. Based on the IDS's flow result, the IPS processes the captured flow according to the prevention strategies. They have performed the experiments on the CIC DoS dataset using six ML methods and achieved $95 \%$ accuracy with a very low False Alarm Rate (FAR).

To identify the Man In The Middle (MITM) attack, Sebbar et al. [148] propose a model using the RF algorithm that selects nodes based on the context to identify ARP resolution instances and any eavesdropping or poisoning within the network. Any connection request with TTL value greater than 200 milliseconds is considered as an attempt of an MITM attack. Nodes within a specified period will only be accepted. Then, using some preestablished policies and the TTL delay, the decision regarding any connection will be made. Any connection requests exceeding the delay will be considered as an attack, and the system cut off the connection to that node preventing any approval and verification process for that node.

Aiken and Scott-Hayward propose an IDS [149] for SDN by developing an anomaly-based network IDS module named Neptune motivated by Athena [150] and an adversarial test module as Hydra. Using Hydra, the authors have tested the detection accuracy of Neptune by attacking with SYN-Flood DDoS attack. Neptune uses SL on flow statistics to train and categorize live traffic. They have evaluated the model's detection accuracy using four ML classifiers, where RF achieved the best outcome.

To assess the performance of different ML algorithms for identifying DDoS attacks in SDN, Meti et al. [151] presented their experiment results in real-time data. The dataset is created from real-time TCP traffic between the experiment lab and the outside world. Mininet is used to create SDN topology. For classification, they have used SVM, NB, and ANN ML models. Their experiment shows that SVM and ANN both achieved $80 \%$ accuracy, however the result substantially varied in precision and recall calculation.

Santos et al. [152] analyze the performance of four ML methods, MLP, DT, RF, SVM, in detecting DDoS attacks in the Mininet simulated SDN environment. They have used the scapy tool to produce malicious traffic for the attack. The analysis was done concerning accuracy in the detection of DDoS attacks and processing time. They also provided a feature analysis to find the best features for training the model. RF algorithm achieved the best accuracy, whereas DT achieved the best efficiency in terms of processing time. Some other studies [153]-[155] mainly adopted SVM-based IDS solution for SDNs.

Table $\mathrm{V}$ presents a tabular summarization of the aforementioned reviewed articles that depicts the objective of the study, adopted SL based Shallow ML algorithms for building
IDS solution, used dataset, core feature selection, chosen SDN controller, and the attack classification type along with their names. In Table XI, we have also provided the summary of each reviewed articles performance based on ML-DL algorithms learning categories, and evaluation metrics.

\section{B. Unsupervised Learning (UL) Based Models}

UL-based models are used when the class label is unknown. In most of the IDS, the UL-based algorithm is used for cluster analysis. As shown in Fig. 10, UL-based algorithms do not require supervision and are trained using unlabeled data. Rather, it investigates the trends and patterns on its own. The unsupervised model's goal is to identify the group and categorized them according to their shared attributes. The most popular example of unsupervised models that are being used for intrusion detection are K-Means and SOM algorithm.

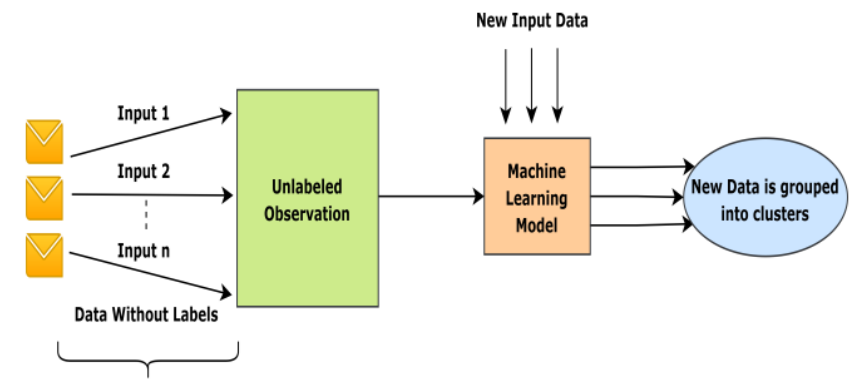

Fig. 10. Unsupervised Learning (UL) method structure.

\section{K-Means Clustering}

$\mathrm{K}-\mathrm{Means}$ clustering method is used to find groups from given dataset where several groups are represented by variable $\mathrm{K}$. Generally, centroids are picked randomly, and K-clusters are formed. It works iteratively to assign a data point to a particular cluster. At the end of the iterations, each data point is clustered according to its feature similarity. Each time cluster center (mean of a cluster) is updated; Iterated until the criterion function converges. Usually, the squared error measure is used through Eq. 6.

$$
\mathrm{E}=\sum_{\mathrm{i}=1}^{\mathrm{k}} \sum_{x \in \mathrm{c}_{\mathrm{i}}}\left|\mathrm{x}-\mathrm{m}_{\mathrm{i}}\right|^{2}
$$

Here, $E=$ sum of the squared errors of all objects, $x=$ point in space representing a given object, and $m_{i}=$ mean of cluster $c_{i}$. The findings of the K-Means method are the K cluster centroids that could be utilized for labeling novel data.

\section{Self-Organizing Map (SOM)}

SOM is an UL method that creates a nonlinear mapping of a high-dimensional data manifold on a regular, low-dimensional output space [156]. Using dimensionality reduction, they can cluster large amounts of data. Compared to the performance of other clustering algorithms, such as K-Means, the SOM output allows for simple visualization. 


\begin{tabular}{|c|c|c|c|c|c|c|c|c|c|}
\hline \multicolumn{10}{|c|}{$\begin{array}{l}\text { TABLE V } \\
\text {-BASED SHA }\end{array}$} \\
\hline Objective & Algorithm & Dataset & Controller & Testbed & $\begin{array}{c}\text { Features Selection } \\
\text { (FS) }\end{array}$ & FS Approach & $\begin{array}{c}\text { Classification } \\
\text { Type }\end{array}$ & IDS Domain & Ref. \\
\hline $\begin{array}{l}\text { Deep packet inspection } \\
\text { based malicious payload } \\
\text { identification in SDN. }\end{array}$ & $\begin{array}{l}\text { DT, RF, KNN, } \\
\text { SVM, LR, } \\
\text { Multinomial-NB }\end{array}$ & $\begin{array}{l}\text { CTU-BOTNET [157] } \\
\text { GitHub Payloads } \\
\text { HTTP CSTC } 2010\end{array}$ & RYU [29] & Mininet & $\begin{array}{l}\text { Term frequency and } \\
\text { Linguistic features. }\end{array}$ & Manual Selection & $\begin{array}{c}\text { Binary } \\
\text { Classification: } \\
\text { Normal class and } \\
\text { Anomalous } \\
\text { class. }\end{array}$ & $\begin{array}{l}\text { Packet-based } \\
\text { Flow-based }\end{array}$ & [121] \\
\hline $\begin{array}{l}\text { Develop a QoS-aware } \\
\text { traffic classification } \\
\text { framework using ML and } \\
\text { DPI in SDN environment. }\end{array}$ & $\begin{array}{c}\text { Tri-Training } \\
\text { Mechanism } \\
\text { (SVM, KNN, } \\
\text { NB) }\end{array}$ & Not Mentioned & $\begin{array}{c}\text { Not } \\
\text { Mentioned }\end{array}$ & Not Mentioned & 8 Features - Flow-based & Eigenvalue-based & $\begin{array}{c}\text { Binary } \\
\text { Classification: } \\
\text { Normal class and } \\
\text { Attack class. } \\
\end{array}$ & Flow-based & [122] \\
\hline $\begin{array}{l}\text { ML-based recognition of } \\
\text { ransomware in SDN }\end{array}$ & $\mathrm{RF}$ & $\begin{array}{c}\text { Malware Traffic } \\
\text { Analysis [158] }\end{array}$ & $\begin{array}{c}\text { Not } \\
\text { Mentioned }\end{array}$ & Not Mentioned & $\begin{array}{c}28 \text { Features -Flow- } \\
\text { based }\end{array}$ & $\begin{array}{l}\text { Manual selection } \\
\text { based on } \\
\text { measuring packet } \\
\text { interarrival times. }\end{array}$ & $\begin{array}{c}\text { Binary } \\
\text { Classification: } \\
\text { Clean class and } \\
\text { Ransomware } \\
\text { class. }\end{array}$ & $\begin{array}{l}\text { Packet-based } \\
\text { Flow-based }\end{array}$ & [127] \\
\hline $\begin{array}{c}\text { Detection and Mitigation of } \\
\text { DDoS attack in SDN though } \\
\phi \text {-entropy incorporating } \\
\text { with SVM and KNN } \\
\text { classifier. }\end{array}$ & KNN, SVM & $\begin{array}{c}\text { Synthetic Data } \\
\text { generated using } \\
\text { Hping3 and Nping. }\end{array}$ & $\begin{array}{c}\text { Not } \\
\text { Mentioned }\end{array}$ & Not Mentioned & $\begin{array}{c}\text { Average byte and } \\
\text { duration. }\end{array}$ & $\begin{array}{c}\text { Shannon entropy } \\
\& \phi \text {-entropy }\end{array}$ & $\begin{array}{c}\text { Multiclass } \\
\text { Classification: } \\
\text { Normal, SYN, } \\
\text { ICMP, UDP, } \\
\text { ACK, TCP } \\
\text { Connection, and } \\
\text { Flash event. }\end{array}$ & $\begin{array}{c}\text { Flow-based } \\
\text { Statistics- } \\
\text { based }\end{array}$ & [159] \\
\hline $\begin{array}{l}\text { Traffic awareness-based } \\
\text { IDS to maintain regulated } \\
\text { traffic in SDN. }\end{array}$ & $\mathrm{RF}$ & KDD'99 & $\begin{array}{c}\text { Floodlight } \\
\text { [33] }\end{array}$ & Mininet & $\begin{array}{l}10 \text { Features: Duration, } \\
\text { Service, Src. bytes, Dst. } \\
\text { bytes, Protocol type, } \\
\text { Flag, Land, Wrong } \\
\text { fragment, Urgent and } \\
\text { Hot. }\end{array}$ & $\begin{array}{c}\text { RF } \\
\text { Based on vote } \\
\text { count of correct } \\
\text { classes. }\end{array}$ & $\begin{array}{c}\text { Binary } \\
\text { Classification: } \\
\text { Normal class and } \\
\text { Anomaly class. }\end{array}$ & Flow-based & [128] \\
\hline $\begin{array}{l}\text { Anomalous traffic detection } \\
\text { and flow control } \\
\text { mechanism. }\end{array}$ & SVM & $\begin{array}{l}\text { Self-Collected data } \\
\text { from network traffic. }\end{array}$ & Floodlight & Not Mentioned & $\begin{array}{c}2 \text { Features: IP address } \\
\text { and Transport port } \\
\text { number. }\end{array}$ & $\begin{array}{l}\text { Based on entropy } \\
\text { analysis }\end{array}$ & $\begin{array}{c}\text { Binary } \\
\text { Classification: } \\
\text { Normal class and } \\
\text { Anomaly class. }\end{array}$ & Flow-based & [129] \\
\hline $\begin{array}{l}\text { Identify malicious hosts and } \\
\text { provide advance security. }\end{array}$ & $\begin{array}{c}\text { DT, NB, } \\
\text { Decision Table, } \\
\text { BayesNet }\end{array}$ & Log data & $\begin{array}{c}\text { Not } \\
\text { Mentioned }\end{array}$ & Not Mentioned & $\begin{array}{l}3 \text { Features: Invader IP, } \\
\text { Compromised host and } \\
\text { No. of efforts in an } \\
\text { outbreak. }\end{array}$ & Not Mentioned & $\begin{array}{c}\text { Binary } \\
\text { Classification: } \\
\text { Normal and } \\
\text { Attack. }\end{array}$ & Log-based & [84] \\
\hline $\begin{array}{l}\text { Recognition of DDoS } \\
\text { anomaly flows in SDN. }\end{array}$ & KNN & $\begin{array}{l}\text { Self-Collected data } \\
\text { From multiple data } \\
\text { centers. }\end{array}$ & RYU & Mininet & $\begin{array}{c}\text { 11 Features: Duration, } \\
\text { Protocol type, Src/Dst. } \\
\text { bytes, Service and } \\
\text { Server count, Server } \\
\text { SYN error Rate, Dst. } \\
\text { host count, Dst. host } \\
\text { server count, Dst. host } \\
\text { SYN error rate and Dst. } \\
\text { host server REJ error } \\
\text { rate. }\end{array}$ & Not Mentioned & $\begin{array}{c}\text { Binary } \\
\text { Classification: } \\
\text { Normal and } \\
\text { DDoS. }\end{array}$ & Flow-based & [130] \\
\hline $\begin{array}{l}\text { Identification of usual and } \\
\text { anomalous flow of data } \\
\text { transmission to detect the } \\
\text { intruder anomalies. }\end{array}$ & $\begin{array}{c}\text { RF, NB, } \\
\text { BayesNet, Part }\end{array}$ & NSL-KDD & Floodlight & Mininet & $\begin{array}{l}\text { Nominal, Numeric and } \\
\text { Binary Feature Set. }\end{array}$ & $\begin{array}{l}\text { Information Gain } \\
\text { ratio }\end{array}$ & $\begin{array}{l}\text { Multiclass } \\
\text { Classification: } \\
\text { Normal, DoS, } \\
\text { R2L, Probe, } \\
\text { U2R, and } \\
\text { Unknown. }\end{array}$ & Flow-based & [131] \\
\hline $\begin{array}{c}\text { Detection of DDoS attack in } \\
\text { SDN using a meta-heuristic } \\
\text { method. }\end{array}$ & $\begin{array}{l}\text { Fitting Curve, } \\
\text { Pattern } \\
\text { Recognition, } \\
\text { Time Series }\end{array}$ & NSL-KDD & $\begin{array}{c}\text { OpenDaylight } \\
{[32]}\end{array}$ & Mininet & $\begin{array}{l}5 \text { Features: Duration, } \\
\text { Protocol type, Src. } \\
\text { bytes, Dst. bytes, } \\
\text { Service and Server } \\
\text { count }\end{array}$ & Manual Selection & $\begin{array}{l}\text { Multiclass } \\
\text { Classification: } \\
\text { Normal, DoS, } \\
\text { R2L, Probe, } \\
\text { U2R, and } \\
\text { Unknown. }\end{array}$ & Flow-based & [132] \\
\hline $\begin{array}{l}\text { SVM based IDS for SDN in } \\
\text { cloud data center. }\end{array}$ & SVM & DARPA 1998 & RYU & Mininet & $\begin{array}{c}\text { 6 Features: Avg. } \\
\text { number of packets per } \\
\text { flow, bytes per flow, } \\
\text { avg. duration of a flow, } \\
\text { percentage of } \\
\text { symmetric paired flows, } \\
\text { rate of increase in the } \\
\text { number of single flows } \\
\text { and growth of new } \\
\text { ports. }\end{array}$ & Manual Selection & $\begin{array}{c}\text { Multiclass } \\
\text { Classification: } \\
\text { Normal, DoS, } \\
\text { U2R, R2L, and } \\
\text { Probe. }\end{array}$ & $\begin{array}{l}\text { Flow-based } \\
\text { Packet-based } \\
\text { Log-based }\end{array}$ & [133] \\
\hline $\begin{array}{l}\text { Statistical feature analysis } \\
\text { of flow-based IDS in SDN. }\end{array}$ & $\begin{array}{l}\text { SVM, DT, RF, } \\
\text { KNN and } \\
\text { Bagged Tress }\end{array}$ & Not mentioned. & RYU & Not Mentioned & $\begin{array}{c}8 \text { Features: Duration, } \\
\text { Packet count, Byte } \\
\text { count, Src/Dst IP, } \\
\text { Protocol, Src. and Dst. } \\
\text { port. }\end{array}$ & $\begin{array}{c}\text { Feature } \\
\text { aggregation based } \\
\text { on replaying the } \\
\text { gathered flows } \\
\text { using TCP Replay } \\
\text { to the SDN. }\end{array}$ & $\begin{array}{c}\text { Binary } \\
\text { Classification: } \\
\text { Normal class and } \\
\text { Anomaly class. } \\
\text { Multiclass } \\
\text { Classification: } \\
\text { Normal, DoS, } \\
\text { HTTP Brute- } \\
\text { Force, SSH } \\
\text { Brute-Force. }\end{array}$ & Flow-based & [134] \\
\hline $\begin{array}{l}\text { Threat detection using flow } \\
\text { control method in SDN. }\end{array}$ & $\begin{array}{l}\text { SVM, } \\
\text { ID3 DT }\end{array}$ & KDD'99 & RYU & $\begin{array}{c}\text { sFlow Toolkit } \\
{[160]}\end{array}$ & $\begin{array}{c}2 \text { Sets of behavioral } \\
\text { features selected from } \\
30 \text { features. } \\
1^{\text {ST }} \text { Set: } 23 \text { features } \\
2^{\text {nd }} \text { Set: } 29 \text { features }\end{array}$ & Information Gain & $\begin{array}{c}\text { Multiclass } \\
\text { Classification: } \\
\text { Normal, DoS, } \\
\text { R2L, Probe, } \\
\text { U2R. }\end{array}$ & Flow-based & [135] \\
\hline
\end{tabular}




\begin{tabular}{|c|c|c|c|c|c|c|c|c|c|}
\hline \multicolumn{10}{|c|}{ TABLE V (Continued) } \\
\hline Objective & Algorithm & Dataset & Controller & Testbed & Features Selection (FS) & FS Approach & $\begin{array}{c}\text { Classification } \\
\text { Type }\end{array}$ & IDS Domain & Ref. \\
\hline $\begin{array}{c}\text { Analyze flow statistics } \\
\text { and develop an SVM } \\
\text { based real-time DDoS } \\
\text { attack detection and } \\
\text { resistance model. }\end{array}$ & SVM & KDD'99 & RYU & Mininet & $\begin{array}{c}8 \text { Features: Count, Service } \\
\text { Count, Same Service Count, } \\
\text { Dst. Host Count, Dst. Host } \\
\text { Service Count, Dst. Host } \\
\text { Same Service, Same Port } \\
\text { Rate, Dst. Host SYN and } \\
\text { REJ Error Rate. } \\
\end{array}$ & Not Mentioned & $\begin{array}{c}\text { Binary } \\
\text { Classification: } \\
\text { Normal class and } \\
\text { DDoS class. }\end{array}$ & Flow-based & [136] \\
\hline $\begin{array}{l}\text { Investigates New flow } \\
\text { and Misbehavior attack }\end{array}$ & $\begin{array}{l}\text { SVM, } \\
\text { J48, } \\
\text { NB }\end{array}$ & NSL-KDD & POX & Mininet & $\begin{array}{l}25 \text { Features were selected out } \\
\text { of } 41 \text { features using } \\
\text { correlation heuristic function } \\
\text { evaluation. }\end{array}$ & $\begin{array}{c}\text { Ranker, } \\
\text { Genetic and } \\
\text { Greedy } \\
\text { algorithm. }\end{array}$ & $\begin{array}{l}\text { Multiclass } \\
\text { Classification: } \\
\text { Normal, DoS, } \\
\text { R2L, Probe, } \\
\text { U2R. }\end{array}$ & Flow-based & {$[137]$} \\
\hline $\begin{array}{c}\text { Build a Two-level ML } \\
\text { based IDS in SDN. }\end{array}$ & ID3 & Not Mentioned. & $\begin{array}{c}\text { Not } \\
\text { Mentioned. }\end{array}$ & Mininet & Not Mentioned & $\begin{array}{c}\text { Genetic } \\
\text { algorithm }\end{array}$ & Not Mentioned. & $\begin{array}{c}\text { Flow-based } \\
\text { Packet-based }\end{array}$ & [139] \\
\hline $\begin{array}{c}\text { Attack detection is } \\
\text { Software Defined Internet } \\
\text { of Things network using } \\
\text { modified BAT and RF } \\
\text { algorithm. }\end{array}$ & $\begin{array}{l}\text { Modified } \\
\text { BAT, } \\
\text { Modified RF }\end{array}$ & KDD'99 & $\begin{array}{c}\text { Not } \\
\text { Mentioned. }\end{array}$ & Not Mentioned & 32 Features-Flow. & $\begin{array}{l}\text { Modified BAT } \\
\text { algorithm }\end{array}$ & $\begin{array}{l}\text { Multiclass } \\
\text { Classification: } \\
\text { Normal, DoS, } \\
\text { R2L, Probe, } \\
\text { U2R. }\end{array}$ & Flow-based & [141] \\
\hline $\begin{array}{c}\text { ML algorithms } \\
\text { performance analysis } \\
\text { using NSL-KDD dataset } \\
\text { for attack detection in } \\
\text { SDN paradigm. }\end{array}$ & $\begin{array}{l}\text { SVM, } \\
\text { KNN, } \\
\text { DT }\end{array}$ & NSL-KDD & POX & Mininet & $\begin{array}{c}6 \text { Features: Duration, Type of } \\
\text { the protocol, Src/Dst. bytes, } \\
\text { Count, SYN error Rate. }\end{array}$ & $\begin{array}{c}\text { Manual } \\
\text { Selection }\end{array}$ & $\begin{array}{l}\text { Multiclass } \\
\text { Classification: } \\
\text { Normal, DoS, } \\
\text { R2L, Probe, } \\
\text { U2R. }\end{array}$ & Flow-based & [142] \\
\hline $\begin{array}{l}\text { SVM incorporated with } \\
\text { selective IP traceback- } \\
\text { based IDS mechanism for } \\
\text { SDN. }\end{array}$ & SVM & NSL-KDD & RYU & Mininet & $\begin{array}{c}\text { 9 Features: } \\
\text { Duration, Type of the } \\
\text { protocol, Flag, Src/Dst. } \\
\text { bytes, Count, Service, } \\
\text { Srv_count, } \\
\text { Dst_host_same_src_port_rate }\end{array}$ & $\begin{array}{l}\text { Manual } \\
\text { Selection }\end{array}$ & $\begin{array}{c}\text { Binary } \\
\text { Classification: } \\
\text { Normal and } \\
\text { Attack. }\end{array}$ & Flow-based & {$[153]$} \\
\hline $\begin{array}{l}\text { Malware intrusions } \\
\text { detection in SDN using } \\
\text { SVM based IDS. }\end{array}$ & SVM & Not Mentioned. & $\begin{array}{c}\text { Not } \\
\text { Mentioned. }\end{array}$ & Not Mentioned & $\begin{array}{l}7 \text { Features: No. of packets, } \\
\text { No. of bytes, Flow duration, } \\
\text { Byte rate, Avg. length of the } \\
\text { packet, Packet rate, First } \\
\text { packet length. }\end{array}$ & $\begin{array}{c}\text { Ranker } \\
\text { algorithm }\end{array}$ & $\begin{array}{c}\text { Binary } \\
\text { Classification: } \\
\text { Normal and } \\
\text { Malware. }\end{array}$ & Flow-based & [112] \\
\hline $\begin{array}{l}\text { Performance evaluation } \\
\text { of Traditional ML } \\
\text { methods regarding attack } \\
\text { detection in SDN. }\end{array}$ & $\begin{array}{c}\text { SVM, } \\
\text { J48, } \\
\text { NB, } \\
\text { RF }\end{array}$ & NSL-KDD & $\begin{array}{c}\text { Not } \\
\text { Mentioned. }\end{array}$ & Not Mentioned & 20 Features - Flow based. & $\begin{array}{c}\text { t-SNE } \\
\text { algorithm } \\
{[161]}\end{array}$ & $\begin{array}{c}\text { Binary } \\
\text { Classification: } \\
\text { Normal and } \\
\text { Malicious. }\end{array}$ & Flow-based & [143] \\
\hline $\begin{array}{l}\text { DDoS attack (TCP-SYN } \\
\text { and ICMP Flood) } \\
\text { detection in the SDN } \\
\text { enabled ISP networks. }\end{array}$ & $\begin{array}{c}\text { KNN, } \\
\text { XGBoost }\end{array}$ & CAIDA 2007 & POX & Self-constructed & Not Mentioned & $\begin{array}{l}\text { Based on Time } \\
\text { window } \\
\text { monitoring and } \\
\text { entropy } \\
\text { calculation }\end{array}$ & $\begin{array}{c}\text { Binary } \\
\text { Classification: } \\
\text { Normal and } \\
\text { DDoS. }\end{array}$ & $\begin{array}{c}\text { Flow-based } \\
\text { Statistics- } \\
\text { based }\end{array}$ & [144] \\
\hline $\begin{array}{c}\text { Building a robust } \\
\text { classification system to } \\
\text { detect DDoS attack by } \\
\text { reducing the dependency } \\
\text { on outdated data. }\end{array}$ & $\begin{array}{l}\text { J48, BayesNet, } \\
\text { Random Tree, } \\
\text { REPTree, NB, } \\
\text { LR. }\end{array}$ & $\begin{array}{l}\text { UNB-ISCX, CTU } \\
13, \text { ISOT. }\end{array}$ & Floodlight & Mininet & $\begin{array}{c}\text { 15 Features: } \\
\text { Statistical, IP, TCP, UDP and } \\
\text { Raw Feature Set. }\end{array}$ & $\begin{array}{c}\text { Manual } \\
\text { Selection based } \\
\text { on neighboring } \\
\text { nodes. }\end{array}$ & $\begin{array}{c}\text { Binary } \\
\text { Classification: } \\
\text { Normal and } \\
\text { DDoS. }\end{array}$ & Flow-based & [146] \\
\hline $\begin{array}{c}\text { Low-rate DDoS attack } \\
\text { detection using ONOS } \\
\text { controller and ML } \\
\text { methods. }\end{array}$ & $\begin{array}{c}\text { J48, REPTree, } \\
\text { RF Random } \\
\text { Tree, SVM, } \\
\text { MLP }\end{array}$ & CIC-DDoS-2019 & ONOS [34] & Mininet & 44 Features-Flow. & $\begin{array}{c}\text { Manual } \\
\text { selection }\end{array}$ & $\begin{array}{c}\text { Binary } \\
\text { Classification: } \\
\text { Normal and } \\
\text { DDoS. }\end{array}$ & Flow-based & [147] \\
\hline $\begin{array}{l}\text { Man In the Middle Attack } \\
\text { recognition in the SDN. }\end{array}$ & $\mathrm{RF}$ & $\begin{array}{l}\text { Self-Collected from } \\
\text { SDN traffic. }\end{array}$ & OpenDaylight & Mininet & Not Mentioned & Not Mentioned & $\begin{array}{c}\text { Binary } \\
\text { Classification: } \\
\text { Normal and } \\
\text { MITM. }\end{array}$ & $\begin{array}{c}\text { Flow-based } \\
\text { Session- } \\
\text { based }\end{array}$ & [148] \\
\hline $\begin{array}{l}\text { SYN-Flood DDoS attack } \\
\text { detection in SDN. }\end{array}$ & $\begin{array}{l}\text { RF, LR, KNN, } \\
\text { SVM }\end{array}$ & $\begin{array}{c}\text { CICIDS, DARPA } \\
2009\end{array}$ & Faucet [162] & Self-Constructed & $\begin{array}{l}15 \text { Features - Packet header } \\
\text { and Stateful Features. }\end{array}$ & $\begin{array}{c}\text { Ranker } \\
\text { algorithm }\end{array}$ & $\begin{array}{c}\text { Binary } \\
\text { Classification: } \\
\text { Benign and } \\
\text { Malicious. } \\
\end{array}$ & $\begin{array}{l}\text { Flow-based } \\
\text { Packet-based }\end{array}$ & [149] \\
\hline $\begin{array}{c}\text { Evaluating performance } \\
\text { of different } \mathrm{ML} \\
\text { algorithms }\end{array}$ & $\begin{array}{l}\text { NB, SVM, } \\
\text { ANN }\end{array}$ & $\begin{array}{c}\text { Self-Constructed } \\
\text { from Real-Time } \\
\text { Traffic. }\end{array}$ & $\begin{array}{c}\text { Not } \\
\text { Mentioned }\end{array}$ & Mininet & $\begin{array}{c}\text { Two Features: } \\
\text { Host time, No. of requests. }\end{array}$ & Not Mentioned & $\begin{array}{c}\text { Binary } \\
\text { Classification: } \\
\text { Normal and } \\
\text { DDoS. }\end{array}$ & Flow-based & [151] \\
\hline $\begin{array}{c}\text { Performance analysis of } \\
\text { four ML algorithms to } \\
\text { identify DDoS attack in } \\
\text { the SDN. }\end{array}$ & $\begin{array}{l}\text { MLP, DT, } \\
\text { SVM, RF }\end{array}$ & Simulated Data. & POX & Mininet & 23 Features - Flow. & $\begin{array}{c}\text { Experimental } \\
\text { Trail-based }\end{array}$ & $\begin{array}{c}\text { Binary } \\
\text { Classification: } \\
\text { Normal and } \\
\text { DDoS. }\end{array}$ & Flow-based & [152] \\
\hline $\begin{array}{c}\text { DDoS Flooding } \\
\text { Occurrence recognition } \\
\text { and mitigation scheme in } \\
\text { SDN. }\end{array}$ & SVM & $\begin{array}{c}\text { Real-Time Traffic } \\
\text { Collected from } \\
\text { home office and } \\
\text { ISP. }\end{array}$ & POX & Mininet & $\begin{array}{c}5 \text { Features: } \\
\text { Source IP, port } \\
\text { Destination IP, port } \\
\text { Protocol }\end{array}$ & $\begin{array}{l}\text { Shannon } \\
\text { Entropy }\end{array}$ & $\begin{array}{c}\text { Binary } \\
\text { Classification: } \\
\text { Normal and } \\
\text { DDoS. }\end{array}$ & Flow-based & [154] \\
\hline $\begin{array}{c}\text { Advanced-SVM-based } \\
\text { DDoS Detection in SDN. }\end{array}$ & SVM & Real-Time Traffic & $\begin{array}{c}\text { Not } \\
\text { Mentioned }\end{array}$ & Not Mentioned & Not Mentioned & $\begin{array}{c}\text { Manual } \\
\text { Selection }\end{array}$ & $\begin{array}{c}\text { Binary } \\
\text { Classification: } \\
\text { Normal and } \\
\text { DDoS. }\end{array}$ & Flow-based & [163] \\
\hline $\begin{array}{c}\text { DDoS attack detection } \\
\text { using feature selection } \\
\text { and ML-based techniques } \\
\text { in SDN. }\end{array}$ & $\begin{array}{l}\text { SVM, ANN, } \\
\text { KNN, NB }\end{array}$ & $\begin{array}{l}\text { Self-Generated } \\
\text { Simulated Data }\end{array}$ & POX & $\begin{array}{l}\text { Self-constructed } \\
\text { using Open } \\
\text { vSwitch. }\end{array}$ & 12 Features - Flow. & $\begin{array}{c}\text { Filter, Wrapper } \\
\text { and } \\
\text { Embedded- } \\
\text { based method. }\end{array}$ & $\begin{array}{c}\text { Multiclass } \\
\text { Classification: } \\
\text { Normal, TCP, } \\
\text { ICMP, and UDP. }\end{array}$ & Flow-based & [155] \\
\hline
\end{tabular}


The initialization of the weight vectors is the first stage in the SOM mapping process. Then, a sample vector is chosen at random, and the map of weight vectors is explored for the weight that best describes that sample. Every weight vector has weights in its immediate vicinity. The chosen weight is rewarded by the ability to become increasingly similar to the randomly picked sample vector. The neighbors of that particular weight are rewarded as well, as they can become increasingly similar to the sample vector chosen, allowing the map to expand and take on new forms. SOM algorithm is extensively used in developing unsupervised learning-based intrusion detection solutions for SDNs [67], [164]-[166].

\section{Hidden Markov Model (HMM)}

The Hidden Markov Model is a probabilistic model based on the Markov processes that have been used in a variety of research fields, including bioinformatics, speech recognition, and network intrusion detection [92], [167]. It enables us to forecast a series of hidden (unknown) states based on a set of observed states. HMMs can be applied to detect complicated internet attacks with a high noise ratio because of the variations in action sequence throughout execution of each identical attack [168].

\section{Unsupervised ML Based IDS in SDN}

In the following, we analyze some of the unsupervised MLbased IDS in SDN.

In a study by Wang et al. [169], HMM is combined with the calculated Renyi entropy of the source and destination IP of the incoming data packets collected by the SDN controller to create an HMM-R scheme that detects low-rate DDoS attacks. For the traffic acquisition, the authors have employed an SDN controller. In order to reduce detection time, the packet_in message is utilized to set the detection period. The authors then used the Renyi entropy as a statistical feature to limit the number of false positives. Finally, the authors employed the proposed HMM-R scheme to define a range of states in the form of probability to identify low-rate DDoS attacks at various rates. Baum-Welch algorithm was used for training the observation sequence data, and viterbi algorithm was utilized for detecting low-rate DDoS attacks in the HMM-R scheme.

The DDoS attack is one of the substantial threats to security in SDN. Braga et al. [170] present a DDoS attack recognition system applied on a NOX controller based SDN. NOX is used for collecting traffic flow features. These features were utilized using the SOM to detect the fake hosts. Their proposed model provides a guard against fake hosts that could produce many requests to exhaust SDN and limit genuine hosts' resources.

Barki et al. [171] design a module-based DDoS attack recognition structure. Their offered IDS has two modules: signature-based IDS detection system and a progressive IDS system. They used KNN, K-Means, NB, and K-Medoids methods for the signature-based IDS. The signature IDS module classifies abnormal traffic and identifies the hosts responsible for generating unusual traffic in the network. The advanced module inspects the packets from suspected hosts and puts a verdict on each host; either it is an authorized host or an irregular host responsible for a DDoS attack. The authors also claimed that the processing time is reduced as advanced modules only check the suspected hosts among all hosts.

Hurley et al. [167] propose a HMM based IDS that can monitor the network and learn from the evolving nature of the network to detect the probability of intrusion inside the network. The HMM defines the likelihood of intrusion by treating features like packet length, src/dst. port number, src/dst. IP addresses as independent measures.

By creating a virtual testbed for SDN traffic generation, Jankowski and Amanowicz [172] propose an IDS based on SOM and Learning Vector Quantization (LVQ) methods. They have also experimented with multiple improved versions of both the algorithm like Multipass SOM, Multipass LVQ-1 and Hierarchical LVQ-1 with an average True Positive Rate (TPR) value of more than $94 \%$.

Table VI presents a tabular summary of the aforementioned reviewed articles that depicts the objective of the study, adopted UL based shallow ML algorithms for building IDS solution, used dataset for the task, core feature selection, chosen SDN controller, and the attack classification type, corresponding IDS domain along with their simulated testbed.

In the next section we discuss those studies which have used RL-based algorithms to develop IDS solutions specific to SDN.

\section{Reinforcement Learning (RL) Based Models}

Reinforcement Learning (RL) is one of three primary ML paradigms, next to supervised and unsupervised learning. RL is concerned about how intelligent agents can achieve a goal in an unknown, potentially intricate environment in order to optimize the concept of total collective reward. RL can be used to solve problems where notable domain information is either inaccessible or prohibitively expensive [173]. In most cases, a function approximator, such as Neural Network, SVM, etc. is used to map state to value. For IDS, designing a reward feature associated with the detection of intrusions is incredibly challenging because there is no automated approach to distinguish intrusions from the normal traffic flow. Algorithms such as Q-learning, Deep-Q Network (DQN), and Proximal Policy Optimization (PPO) are mostly used in RL-based IDS for SDNs. Q-learning is an off-policy RL algorithm that determines the optimum course of action given the present situation [174]. Because the Q-learning function learns from its actions and isn't reliant on the existing policy, it's termed off-policy. 


\begin{tabular}{|c|c|c|c|c|c|c|c|c|c|}
\hline \multicolumn{10}{|c|}{ TABLE VI } \\
\hline Objective & Algorithm & Dataset & Controller & Testbed & Features Selection (FS) & FS Approach & $\begin{array}{c}\text { Classification } \\
\text { Type }\end{array}$ & $\begin{array}{c}\text { IDS } \\
\text { Domain }\end{array}$ & Ref. \\
\hline $\begin{array}{c}\text { Detecting low-rate } \\
\text { DDoS attacks using } \\
\text { HMM at different rates } \\
\text { in the SDN. }\end{array}$ & $\begin{array}{c}\text { HMM, } \\
\text { SOM, } \\
\text { KNN, } \\
\text { Back-Propagation }\end{array}$ & $\begin{array}{c}\text { Data packets } \\
\text { collected from } \\
\text { synthetically } \\
\text { created data center. }\end{array}$ & POX & Mininet & $\begin{array}{l}\text { Source and Destination IP } \\
\text { addresses. }\end{array}$ & Renyi entropy & $\begin{array}{c}\text { Binary } \\
\text { Classification: } \\
\text { Normal and } \\
\text { DDoS. }\end{array}$ & $\begin{array}{c}\text { Flow-based } \\
\text { Statistics- } \\
\text { based }\end{array}$ & [169] \\
\hline $\begin{array}{l}\text { DDoS attack } \\
\text { recognition. }\end{array}$ & SOM & $\begin{array}{l}\text { Self-Collected data } \\
\text { from network } \\
\text { traffic. }\end{array}$ & NOX & Not Mentioned & $\begin{array}{c}\text { Per flow average of } \\
\text { packets, bytes, duration, } \\
\text { and percentage of pair- } \\
\text { flows. }\end{array}$ & Self-Extracted & $\begin{array}{c}\text { Binary } \\
\text { Classification: } \\
\text { Normal and } \\
\text { DDoS. }\end{array}$ & $\begin{array}{l}\text { Flow-based } \\
\text { Statistics- } \\
\text { based }\end{array}$ & [170] \\
\hline $\begin{array}{l}\text { DDoS attack recognition } \\
\text { and identify the attacker } \\
\text { hosts. }\end{array}$ & $\begin{array}{c}\text { K-Means, } \\
\text { K-Medoids, } \\
\text { NB, } \\
\text { KNN }\end{array}$ & $\begin{array}{l}\text { Self-Collected data } \\
\text { from network } \\
\text { traffic. }\end{array}$ & RYU & Mininet & Not Mentioned. & Self-Extracted & $\begin{array}{c}\text { Binary } \\
\text { classification: } \\
\text { Normal and } \\
\text { DDoS. }\end{array}$ & $\begin{array}{l}\text { Flow-based } \\
\text { Statistics- } \\
\text { based } \\
\text { Log-based }\end{array}$ & [171] \\
\hline $\begin{array}{l}\text { Develop an HMM-based } \\
\text { IDS for SDN. }\end{array}$ & HMM & $\begin{array}{l}\text { Self-collected data } \\
\text { from } \\
\text { network traffic. }\end{array}$ & Floodlight & Mininet & $\begin{array}{l}\text { Packet length, Src and Dst. } \\
\text { port number, Src. and Dst. } \\
\text { IP addresses. }\end{array}$ & $\begin{array}{c}\text { Manual } \\
\text { Selection }\end{array}$ & $\begin{array}{c}\text { Binary } \\
\text { Classification: } \\
\text { Normal and } \\
\text { Anomaly. }\end{array}$ & Flow-based & [167] \\
\hline $\begin{array}{l}\text { Network-based DDoS } \\
\text { attack recognition in } \\
\text { SDN environment. }\end{array}$ & SOM & Simulated data. & Floodlight & NS-3 & Not Mentioned. & $\begin{array}{c}\text { Manual } \\
\text { Selection }\end{array}$ & $\begin{array}{c}\text { Binary } \\
\text { Classification: } \\
\text { Normal and } \\
\text { DDoS. }\end{array}$ & Flow-based & [67] \\
\hline $\begin{array}{l}\text { Competence Evaluation } \\
\text { of ML based algorithms } \\
\text { in IDS for SDN. }\end{array}$ & $\begin{array}{l}\text { SOM, } \\
\text { LVQ }\end{array}$ & $\begin{array}{l}\text { Self-collected from } \\
\text { a virtual testbed. }\end{array}$ & OpenDaylight & Mininet & $\begin{array}{l}9 \text { Features: Packet count in } \\
\text { a flow, Src. TCP/UDP port, } \\
\text { Byte's count in a flow, Dst. } \\
\text { TCP/UDP port, Duration, } \\
\text { Flows with diverse ports } \\
\text { from Src/Dst. hosts, Flow } \\
\text { rate to the host, and Single } \\
\text { flow rate to the host. }\end{array}$ & Not Mentioned & $\begin{array}{c}\text { Multiclass } \\
\text { Classification: } \\
\text { Normal, DoS, } \\
\text { R2L, Probe, } \\
\text { U2R. }\end{array}$ & $\begin{array}{l}\text { Statistics- } \\
\text { based } \\
\text { Flow-based }\end{array}$ & [172] \\
\hline
\end{tabular}

Rather than estimating the value function with a greedy approach, Q-learning updates its value functions using equations such as the Bellman equation. The letter ' $Q$ ' stands for quality in Q-learning. In this situation, quality refers to how valuable a specific action is in obtaining a future reward. By combining Qlearning with DNN, V. Mnih et al. [175] proposed the DQN algorithm that operates within discrete action spaces. A DQN agent is a value-based RL-based agent that teaches a critic to predict future rewards or returns. The OpenAI team launched the PPO algorithm in 2017 [176], and it immediately became one of the most popular RL approaches, overtaking Deep-Q learning. It is a policy gradient method that is used to train policies in a variety of prominent RL-based applications [177]. It involves gathering a small sample of the environment and using that sample to update the decision-making policy.

Sampaio et al. [178] emphasize the use of RL and Network Function Virtualization (NFV) architecture for detecting anomalies in SDN. The authors suggested that network metrics be collected and categorized into profiles, each with a series of actions that handle functions through RL, NFV, and an SDN controller. Based on the rewards for individual actions, authors have set up the anomaly detection policies. They have load balanced the traffic flow in an online manner without any supervision. Their model also detects honeypots by initiating a Linux bot running with secure shell and telnet service.

Zolotukhin et al. [179] propose an RL-based technique for attack detection and real-time dynamic reconfiguration of the network by redirecting the SDN flows to multiple security middle boxes. They have calculated the maximum number of packets sent to a host and created a set of rules defining threshold values for different processes. The snort virtual appliance detects any amount exceeding the threshold volume. They adopted a centroid-based clustering method to detect anomalous payload.
They have collected regular traffic from the network and divided them into numerous clusters using K-Means, SOM, FCM algorithm to train the RL agent. For RL, they have used DQN and PPO.

A Neural Fitted Q-Learning agent-based threat detection mechanism named ATMoS was proposed by Akbari et al. [180] using OpenDayLight, Snort, Docker, etc. This model's three key components are profiling of the behavior of the host, autonomous management for the RL agent, and SDN infrastructure. APT and TCP SYN-flood attacks were detected using deploying malicious hosts and variations of benign hosts in the simulated testbed.

Q-Learning is also explored by Phan et al. in their DoS defense framework named Q-Mind [181] in SDN. The Q- Learningbased agent controls the anomaly classification system based on SVM, SOM, and RF. A module named application operator and scheduler selects the optimal features from the collected data and chooses appropriate ML algorithms to classify each source IP address into a normal or malicious one.

Table VII presents a tabular summary of the aforementioned reviewed articles that depicts the objective of the study, adopted RL based ML-DL algorithms for building IDS solution, used dataset for the task, core feature selection, chosen SDN controller, and the attack classification type, corresponding IDS domain along with their simulated testbed. In the next section, we discuss those studies which have used DL-based algorithms to develop IDS solutions specific to SDN.

\section{DEEP LEARNING MODELS BASED IDS IN SDN}

Feature-based shallow ML detection schemes are incredibly resource-intensive, and their efficacy in intrusion detection performances in large-scale SDN is not very reliable [182]. Recently, DL-based models have gained popularity over traditional ML models because of higher accuracy and precision. 


\begin{tabular}{|c|c|c|c|c|c|c|c|c|c|}
\hline \multicolumn{10}{|c|}{$\begin{array}{r}\text { TABLE VII } \\
\text { NT LEARNING-BA }\end{array}$} \\
\hline Objective & Algorithm & Dataset & Controller & Testbed & Features Selection (FS) & FS Approach & $\begin{array}{c}\text { Classification } \\
\text { Type }\end{array}$ & IDS Domain & Ref. \\
\hline $\begin{array}{l}\text { Usage of RL and NFV in } \\
\text { SDN for Anomaly } \\
\text { Detection. }\end{array}$ & $\overline{R L}$ & $\begin{array}{c}\text { Self-generated } \\
\text { using Mininet } \\
\text { platform along with } \\
\text { Docker. }\end{array}$ & POX & Mininet & Not Mentioned. & $\begin{array}{c}\text { Not } \\
\text { Mentioned. }\end{array}$ & Not Mentioned & Flow-based & [178] \\
\hline $\begin{array}{l}\text { Attack detection and } \\
\text { alleviation using SDN } \\
\text { flow through redirection } \\
\text { into virtual appliances. }\end{array}$ & $\begin{array}{l}\text { DQN, } \\
\text { PPO }\end{array}$ & $\begin{array}{l}\text { Self-generated } \\
\text { using regular } \\
\text { network traffic. }\end{array}$ & OpenDaylight & Not Mentioned & $\begin{array}{l}\text { Port, TCP flags, Packet size, } \\
\text { Packet count, Security logs } \\
\text { and alerts, Protocol, and } \\
\text { SDN flows. }\end{array}$ & $\begin{array}{c}\text { Manual } \\
\text { Selection. }\end{array}$ & $\begin{array}{c}\text { Multiclass } \\
\text { Classification: } \\
\text { SSH password } \\
\text { brute-force, } \\
\text { Slowloris DDoS, } \\
\text { DNS Tunneling. }\end{array}$ & $\begin{array}{c}\text { Flow-based } \\
\text { Packet-based }\end{array}$ & [179] \\
\hline $\begin{array}{l}\text { TCP SYN-flood and } \\
\text { Advanced Persistent } \\
\text { Threats (APT) detection } \\
\text { using RL based agent. }\end{array}$ & $\begin{array}{c}\text { Neural Fitted } \\
\text { Q-Learning }\end{array}$ & Simulated Traffic. & OpenDaylight & Mininet & Not Mentioned. & $\begin{array}{c}\text { Not } \\
\text { Mentioned. }\end{array}$ & $\begin{array}{c}\text { Binary } \\
\text { Classification: } \\
\text { TCP SYN-flood } \\
\text { class and APT } \\
\text { attack. }\end{array}$ & Flow-based & [180] \\
\hline $\begin{array}{l}\text { Develops a Defense } \\
\text { mechanism for DoS } \\
\text { attack by incorporating } \\
\text { the optimal policy } \\
\text { resulting from the Q- } \\
\text { Learning agent }\end{array}$ & Q-Learning & Simulated Traffic. & ONOS & MaxiNet [183] & $\begin{array}{c}\text { 10 Features: } \\
\text { Average packets/flow, } \\
\text { average packet size/flow, } \\
\text { packet change ratio, flow } \\
\text { change ratio, average } \\
\text { duration/flow, percentage of } \\
\text { pair- flows, growth of } \\
\text { different ports, average flow } \\
\text { inter-arrival time, fraction of } \\
\text { TCP flows over total } \\
\text { incoming flows and entropy } \\
\text { of incoming flows. }\end{array}$ & $\begin{array}{l}\text { PCA with } \\
\text { SVM, } \\
\text { Binary BAT } \\
\text { with RF. }\end{array}$ & $\begin{array}{c}\text { Binary } \\
\text { Classification: } \\
\text { Normal and } \\
\text { DoS. }\end{array}$ & $\begin{array}{l}\text { Flow-based } \\
\text { Statistics- } \\
\text { based }\end{array}$ & [181] \\
\hline $\begin{array}{l}\text { Deep RL-based traffic } \\
\text { sampling for several } \\
\text { traffic analyzers in SDN. }\end{array}$ & $\begin{array}{l}\text { DDPG, } \\
\text { MDP }\end{array}$ & $\begin{array}{c}\text { Generated traffic } \\
\text { using Iperf, and } \\
\text { Hping3 tools. }\end{array}$ & OpenDaylight & Self-Constructed. & Not Mentioned. & $\begin{array}{c}\text { Not } \\
\text { Mentioned. }\end{array}$ & $\begin{array}{c}\text { Binary } \\
\text { Classification: } \\
\text { Normal and } \\
\text { Malicious. }\end{array}$ & $\begin{array}{c}\text { Flow-based } \\
\text { Packet-based }\end{array}$ & [184] \\
\hline
\end{tabular}

In IDS, many authors successfully applied deep learning models for classification tasks. DL-based models generally consist of complex and wide neural networks. DL-based models can extract a feature from the input data and do not require feature engineering. This is one of the significant reasons why DL models are becoming more popular day by day. DL models can also be classified as SL-based models and UL-based models. The most common SL-based DL models are CNN, RNN, DNN, and DBN. On the other hand, RBM, Stacked Auto Encoder, and Generative Adversarial Network (GAN) are the UL-based models.

\section{A. SL Based DL Algorithms}

Below, we briefly discuss some commonly used SL-based DL algorithms and review the existing literature utilizing these algorithms for IDS solution in SDN.

\section{Deep Neural Network (DNN)}

DNN is a feedforward architecture with numerous hidden layers. There is only one hidden layer in a shallow neural network, there are at least two or more hidden layers, as shown in Fig. 11. The performance of DNN is excellent compared to a

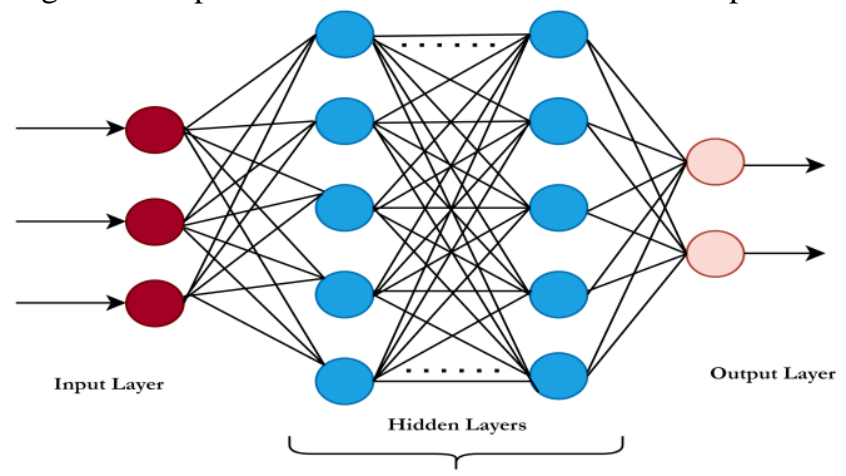

Fig. 11. A generic DNN architecture. single layer neural network because, through various layers, each layer creates features that are conceptual and represent the complex association among those. It also means that the run time complexity and training of the model are computationally costly.

\section{Recurrent Neural Network (RNN)}

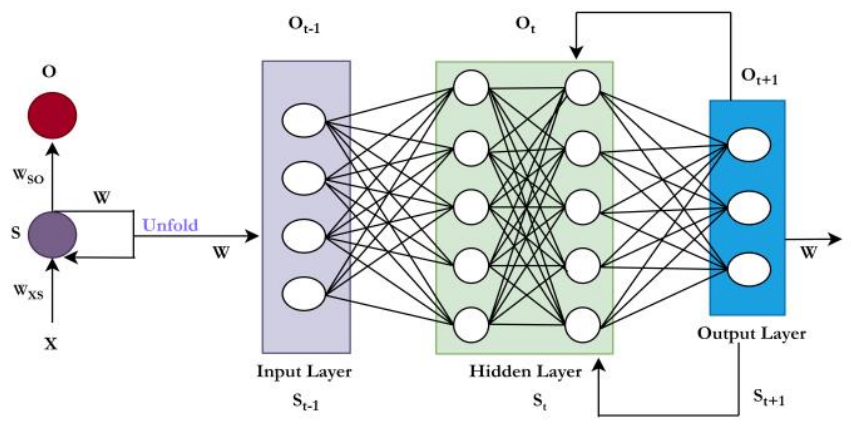

Fig. 12. A generic RNN structure.

RNN is designed to model contextual/sequential data by expanding the functionalities of the conventional feed-forward ANN. RNN consists of the input unit, hidden and output layers, where the memory components are the hidden layers. Every unit in a RNN obtains the current state as well as the previous states to extract contextual information to model the sequential data. Without ambiguity as depicted in Fig. 12, if the network's initial input is $\mathrm{X}$ and the output is $\mathrm{S}$, the network is fed both $\mathrm{S}$ and $\mathrm{X} 1$ (i.e., the next input in the sequential dataset) for the next round of learning. By doing this, the data context (previous inputs) is retained along with the network's training. The distributed hidden layers allow the RNN to store previous information efficiently, making it a powerful tool. LSTM and GRU are the two variants 
of RNN that try to overcome the short-term memory problem of RNN by using a mechanism named as Gates. Gates are basically neural networks that control the flow of data through the sequence chain.

\section{Convolutional Neural Network (CNN)}

CNNs are neural networks with one or more convolutional layers that are primarily used for image processing, detection, segmentation, classification-related tasks. Convolution and pooling are two primary procedures that are often present in CNN. The convolution operation with multiple filters is capable of extracting features (i.e., feature map) from the dataset while preserving their spatial information. Pooling, also known as subsampling, is a technique for reducing the dimensionality of extracted features (feature maps) created by the convolution operation. A typical CNN architecture is shown in Fig. 13, where every input image is processed through multiple layers of convolution and pooling of different kinds and filters before being passed to the fully connected layers.

\section{Supervised DL BASED IDS In SDN}

Now, we analyze some of the supervised DL-based IDS in SDN.

Tang et al. [185] present a DL scheme for flow based anomaly recognition method in SDN with an input layer, 3 hidden layers and lastly an output layer. The model achieved $75.75 \%$ accuracy in detecting flow traffic-based anomaly.

Tang et al. [186] have extended their previous work [185] by proposing a GRU-RNN facilitated IDS for the SDN paradigm and attained an accuracy of $89 \%$, which is improved by $13.25 \%$ from their previous work. The GRU-RNN acquires the long-term contextual representations better, which yields higher anomaly detection rate.

A DL-based botnet detection method is presented by Maeda et al. [187]. The proposed system initially detects the infected host and then separates that host using SDN. For detecting malware, training is conducted using the data obtained from the botnet traffic collected on the traditional network and afterward testing the detection performance. Botnet traffic is retransmitted to isolate a bot-infected device in the SDN, and connectivity with the source IP defined by the ML classifier is clogged and secluded.

DeepIDS, a flow based Deep Learning based IDS in SDN model is proposed by Tang et al. [188]. Using DNN and GRU-RNN they have implemented the DeepIDS in a POX controller. They have also assessed their model in terms of resource utilization, latency and throughput.

Albahar [189] presents an RNN based DL-based model on a novel regularization method called RNN-SDR by decaying the weights according to the calculated standard deviation of weight matrices and then matches the result through its predecessor. The proposed system is a 3-part framework collects flow information at stage-1. At stage-2, using the novel regularization method it detects anomaly, and based on the detection at stage-2, the mitigation module (i.e., stage-3) drops or forwards the packet.

Li et al. [190] propose a DL model to distinguish DDoS attacks in the paradigm of SDN. Their system is comprised of an input layer, forward and recursive layer, a fully connected hidden layer, and finally, the output layer. In their model, they have made use of RNN, LSTM, and CNN. They have used the time window concept for data inputting to the model.

Boukria and Guerroumi [191] propose a DNN-based IDS solution using the CICIDS2017 dataset in a Mininet environment set up using an ONOS controller [192]. Letteri et al. [193] also proposed a MLP-based botnet detection method in SDN with a subset of SDN-specific HogZilla dataset.

Kurochkin and Volkov [194] propose a GRU-RNN based model using a more advanced and practical attack-oriented dataset named CSE-CIC-IDS2018. The model achieved an F1score of 1.00 in detecting DDoS attacks. However, it yields a low detection score in web and infiltration labeled attacks.

Novaes et al. [195] propose an anomaly recognition and mitigation oriented modular system for detecting port scan and DDoS attacks by utilizing LSTM and Fuzzy inference logic algorithm in the SDN environment. The authors quantified the network attribute using the entropy measurement and then used LSTM to predict the pattern of each feature of normal traffic [12]. Its $1^{\text {st }}$ module is the classification module that uses LSTM to predict ordinary network traffic activity. The $2^{\text {nd }}$ module is responsible for detecting anomalous activities, and it uses the BCI theorem to dynamically create a normality threshold, followed by Fuzzy logic to classify the presence of an anomaly at a specific point in the analysis. The system's third module is in charge of mitigating observed irregularities, intending to minimize the damage caused by an intruder.

Gadze et al. [196] analyze the DL-based models' performance for identifying and mitigating DDoS attacks in SDN. The primary focus of their investigation is to detect UDP, TCP, and ICMP flood attacks. LSTM and CNN have been used to

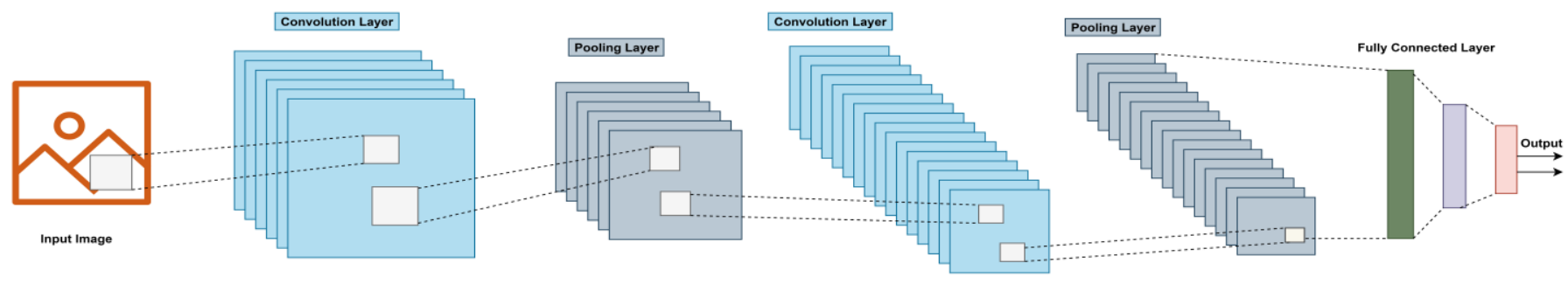

Fig. 13. A generic $\mathrm{CNN}$ architecture. 
demonstrate the DL-based models' performance over classical ML-based models. These models are trained and tested with simulated traffic using the hping 3 tool. Their experiment result shows that LSTM is the best model with an accuracy of $89.63 \%$ under the given circumstances. On the other hand, SVM performs best among linear-based models with an accuracy of $86.85 \%$. Additionally, they also present a comparison that reflects the highest and lowest time taken to detect anomaly using different split ratios.

Ali and Yousaf [197] propose a novel three IDS and prevention system regarding validation of user, packet, and flow in the network. It is a three-phase architecture where authentic IoT users are validated using radio-frequency identification and signature using SHA-256 in the first phase. To encrypt the security credentials, the authors also provided a novel algorithm. In the second phase, using type- 2 fuzzy, packets are classified as normal, dubious, and malicious. Using a CNN-based model, dubious packets are analyzed to predict invader packets and classify the source device correctly in the last phase.

CNN is also explored in the IDS solution in SDN by Kim et al. [198]. For the CNN-based model, the authors have created two kinds of intrusion images, namely RGB and grayscale. They have used KDD CUP 1999 and CSE-CIC-IDS2018, focusing on detecting DoS attacks. They have also experimented with the multiple kernel size, achieving different accuracies. Experiments are conducted using an RNN-based model with five (5) embedding vectors and a sigmoid activation function as hyperparameters and compared the performances between the two models using both binary and multiclass frameworks.

Dey and Rahman [199] investigate two different flow-based IDS methods in the OF controller. The first methods have used the RF classifier algorithm with gain ratio feature selection assessor. The second method has used GRU-LSTM memory with a recursive feature selection elimination method and achieved improved performance from the first one.

\section{B. UL Based DL Algorithms}

Now we briefly discuss some of the commonly used UL-based DL algorithms and review the existing literature utilizing these algorithms for IDS solution in SDN.

\section{AutoEncoder (AE)}

AEs are unsupervised DL technique that learns a compressed distributed representation of the given data mainly focusing on dimensionality reduction. It can learn Non-Linear (NL) transformations using NL activation function in multiple layers providing many representations of diverse dimensions [200], making it an alternative for PCA. It is composed of two symmetric modules namely, encoder and decoder, and a code segment, as shown in Fig. 14. The encoder collects features from the input data, the decoder recreates the data from the features collected. The code segment holds the extracted features. There exist various types of AE's, such as sparse, stacked, and denoising AE's.

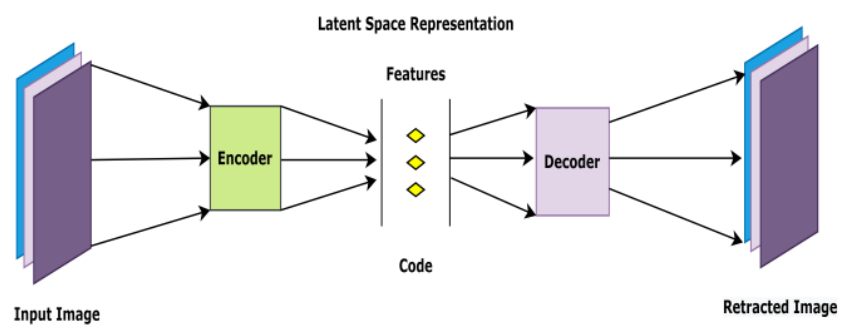

Fig. 14. A generic structure of an autoencoder.

\section{Restricted Boltzmann Machine (RBM)}

RBM is an UL-based method, an energy-based probabilistic neural network having a two-layer network structure with symmetric connectivity and no connections within a layer [201]. One layer is called the visible layer, and the other one is called the hidden layer. Every visible layer node is connected with all other nodes in the hidden layer, as shown in Fig. 15, $\mathrm{V}_{\mathrm{i}}$ represents a visible layer node, $\mathrm{H}_{\mathrm{j}}$ represents a Hidden layer node and $\mathrm{W}_{\mathrm{ij}}$ represents weight between nodes. RBM does not differentiate between the forward and backward propagation, keeping the weights same in both directions.

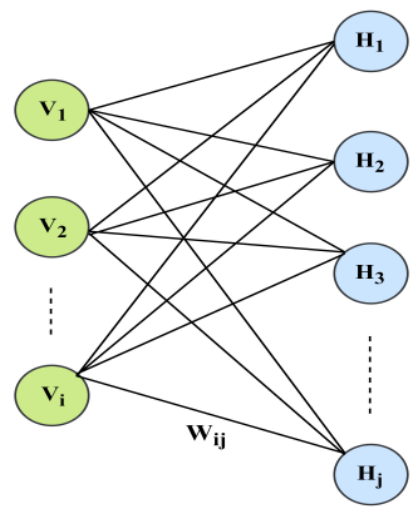

Fig. 15. A generic architecture of an RBM.

\section{Unsupervised DL BASED IDS In SDN}

Now we analyze some of the unsupervised DL-based IDS in SDN.

To create a multi-vector DDoS detection method, Niyaz et al. [202] have used a Stacked Auto-Encoder (SAE). Their model also outlines whether a received traffic is regular or attack oriented. The model reduced the collection of feature attributes extracted through the network traffic headers to increase the recognition performance and lessen the FAR.

An adaptive pooling and sFlow-based sampling scheme for identifying DDoS attacks in IoT network was proposed by Ujjan et al. [203]. They have used a snort-based IDS along with SAE for the detection process between normal and malicious traffic. 
Dawoud et al. [204] propose an unsupervised method for anomaly detection in SDN. At the first stage, they have employed $\mathrm{AE}$ algorithm to minimize the reconstruction error of input samples. In the second stage, clustering was done using K-Means algorithm to cluster normal and abnormal traffic.

By exploiting the collective intelligence between the data and control plane of SDN, Han et al. proposed a framework named Overwatch [205]. It detects DDoS attacks by utilizing autoencoder along with the softmax classifier. The framework also decreases overhead in the southbound interface of SDN. It divides defensive functions collectively between the data and control planes, allowing both planes to detect and defend against DDoS attacks at various levels.

In another study, Novaes et al. [206] have developed a detection and prevention system in SDN based on adversarial training, which leverages the GAN framework to identify DDoS attacks. The adversarial training makes the system less vulnerable to adversarial attacks. They also compared the proposed system's performances to those of other DLbased techniques for detecting DDoS in SDN, such as CNN, LSTM, and MLP. The system is divided into two phases. They assessed the suggested method for detecting UDP flood attacks in an SDN environment with high transmission rates in the first stage. The system performance for identifying DDoS attacks against various applications is evaluated in the second stage.

In [35], Dawoud et al. demonstrate a reliable anomaly detection system for an SDN-based IoT network. IoT devices are positioned at the lowest layer of their proposed architecture, whereas SDN layers such as control and forward layers are located at the top of the IoT devices. The detection system resides at the controller layer and uses RBM to interface directly with the network in their proposed system. According to the authors, the deployment of IDS at the application layer is unable to avoid the controller threats. This approach employs a two-layer RBM network with hidden and visible layers, the latter of which comprises 41 nodes equal to the characteristics of the KDD'99 dataset. The tests of this anomaly detection system revealed that it produces an accuracy rate of $94 \%$, which is greater than the PCA and SVM.

Shu et al. [208] demonstrate an IDS for Vehicular Ad Hoc Networks (VANETs) by installing a distributed SDN controller on each base station to distinguish regular network traffic and malicious network traffic. Using the full network flow information, they used GAN to jointly train numerous SDN controllers for the entire VANET without directly trading their sub-network flows. This IDS approach allows distributed SDN controllers to detect their sub-network flows separately, reducing communication and computation overheads.

Mohanapriya and Shalinie demonstrate a DDoS detection method based on RBM [209]. The suggested approach is divided into two phases: data acquisition and attack detection. Flow collector captures incoming traffic flows from data plane switches and saves them in the database during the data collection step. The hit count and energy consumption rate are determined initially in the attack detection step. The RBM model starts for a particular MAC address if the hit count exceeds the average threshold value and the energy consumption rate is higher than another threshold.

Table VIII presents a tabular summary of the aforementioned reviewed articles that depicts the objective of the study, adopted DL algorithms for building IDS solution, used dataset for the task, core feature selection, chosen SDN controller, and the attack classification type, corresponding IDS domain along with their simulated testbed.

In the next section we discuss those studies which have adopted hybrid approaches to develop IDS solutions specific to SDN.

\begin{tabular}{|c|c|c|c|c|c|c|c|c|c|}
\hline \multicolumn{10}{|c|}{ TABLE VIII } \\
\hline Objective & Algorithm & Dataset & Controller & Testbed & Features Selection (FS) & $\begin{array}{c}\text { FS } \\
\text { Approach }\end{array}$ & Classification Type & $\begin{array}{c}\text { IDS } \\
\text { Domain }\end{array}$ & Ref. \\
\hline $\begin{array}{l}\text { Securing SDN-IoT } \\
\text { network through DL } \\
\text { method. }\end{array}$ & RBM & KDD'99 & $\begin{array}{c}\text { Not } \\
\text { Mentioned }\end{array}$ & Self-Constructed & $\begin{array}{c}\text { All } 41 \text { Features of KDD'99 } \\
\text { dataset. }\end{array}$ & NA & $\begin{array}{c}\text { Binary Classification: } \\
\text { Normal class and } \\
\text { Anomaly class. }\end{array}$ & Flow-based & {$[35]$} \\
\hline $\begin{array}{l}\text { Detecting Intrusion in } \\
\text { SDN using a DNN. }\end{array}$ & DNN & NSL-KDD & $\begin{array}{c}\text { Not } \\
\text { Mentioned. }\end{array}$ & Self-Constructed & $\begin{array}{c}\text { Duration, Protocol type, } \\
\text { Src/Dst. bytes, Server count } \\
\text { and Dst. Host Same Source } \\
\text { Port Rate. }\end{array}$ & $\begin{array}{c}\text { Manual } \\
\text { Selection }\end{array}$ & $\begin{array}{c}\text { Multiclass } \\
\text { Classification: } \\
\text { Normal, DoS, R2L, } \\
\text { Probe, U2R and } \\
\text { Unknown Attacks. }\end{array}$ & Flow-based & [185] \\
\hline $\begin{array}{c}\text { DDoS Attack } \\
\text { Recognition System. }\end{array}$ & SAE & $\begin{array}{c}\text { Collected from a } \\
\text { real network } \\
\text { scenario and a } \\
\text { private network } \\
\text { Testbed. } \\
\end{array}$ & POX & $\begin{array}{l}\text { RIT and GENI } \\
\text { [207] }\end{array}$ & $\begin{array}{l}68 \text { features, extracted for } \\
\text { TCP flow, UDP flows and } \\
\text { ICMP flows. }\end{array}$ & NA & $\begin{array}{c}\text { Multiclass } \\
\text { Classification: } \\
\text { Normal and } 7 \text { class of } \\
\text { DDoS attacks. }\end{array}$ & $\begin{array}{l}\text { Flow-based } \\
\text { Packet- } \\
\text { based }\end{array}$ & [202] \\
\hline $\begin{array}{c}\text { Develop a GRU-RNN } \\
\text { based IDS solution in } \\
\text { SDN. }\end{array}$ & GRU-RNN & NSL-KDD & POX & Not Mentioned & $\begin{array}{c}6 \text { Features - Duration, } \\
\text { Protocol type, Src/Dst. } \\
\text { bytes, Server count, and } \\
\text { Dst. Host Same Src. Port } \\
\text { Rate. } \\
\end{array}$ & NA & $\begin{array}{c}\text { Binary Classification: } \\
\text { Legitimate class and } \\
\text { Anomaly class. }\end{array}$ & Flow-based & [186] \\
\hline
\end{tabular}




\begin{tabular}{|c|c|c|c|c|c|c|c|c|c|}
\hline \multicolumn{10}{|c|}{ TABLE VIII (Continued) } \\
\hline Objective & Algorithm & Dataset & Controller & Testbed & Features Selection (FS) & $\begin{array}{c}\text { FS } \\
\text { Approach }\end{array}$ & Classification Type & $\begin{array}{c}\text { IDS } \\
\text { Domain }\end{array}$ & Ref. \\
\hline $\begin{array}{l}\text { Detecting Botnets in } \\
\text { SDN. }\end{array}$ & MLP & CTU-13 and ISOT & RYU & Not Mentioned & $\begin{array}{l}\text { Statistical, IP, TCP, UDP } \\
\text { and Raw Feature Set. }\end{array}$ & NA & $\begin{array}{c}\text { Binary Classification: } \\
\text { Normal class and Botnet } \\
\text { class. }\end{array}$ & $\begin{array}{l}\text { Session- } \\
\text { based } \\
\text { Flow-based }\end{array}$ & [187] \\
\hline $\begin{array}{l}\text { Deep Learning based } \\
\text { IDS solution in SDN. }\end{array}$ & $\begin{array}{c}\text { DNN } \\
\text { GRU-RNN }\end{array}$ & NSL-KDD & POX & Cbench [210] & $\begin{array}{l}\text { Basic, Traffic and Mixed } \\
\text { Feature Set. }\end{array}$ & NA & $\begin{array}{c}\text { Multiclass } \\
\text { Classification: } \\
\text { Normal, DoS, R2L, } \\
\text { Probe, U2R. }\end{array}$ & Flow-based & [188] \\
\hline $\begin{array}{l}\text { Developing an RNN } \\
\text { based IDS for SDN. }\end{array}$ & RNN & $\begin{array}{c}\text { KDD99 } \\
\text { NSL-KDD and } \\
\text { UNSW-NB15 }\end{array}$ & Beacon [211] & Mininet & $\begin{array}{c}\text { Protocol Type, } \\
\text { Service, Duration and Flag. }\end{array}$ & NA & $\begin{array}{c}\text { Multiclass } \\
\text { Classification: } \\
\text { Normal, DoS, R2L, } \\
\text { Probe, U2R. }\end{array}$ & Flow-based & [189] \\
\hline $\begin{array}{c}\text { DDoS attack } \\
\text { Detection using Deep } \\
\text { Learning Method in } \\
\text { SDN. }\end{array}$ & DNN & ISCX 2012 & $\begin{array}{c}\text { Not } \\
\text { Mentioned. }\end{array}$ & Not Mentioned & 20 Features-Flow based. & NA & $\begin{array}{c}\text { Binary Classification: } \\
\text { Normal class and DDoS } \\
\text { attack. }\end{array}$ & $\begin{array}{c}\text { Flow-based } \\
\text { Statistics- } \\
\text { based }\end{array}$ & [190] \\
\hline $\begin{array}{l}\text { Detection of DDoS } \\
\text { Attack using sFlow and } \\
\text { Adaptive polling } \\
\text { sampling method. }\end{array}$ & SAE & $\begin{array}{c}\text { Self-Generated } \\
\text { using Mininet } \\
\text { Virtual IoT } \\
\text { Topology. }\end{array}$ & RYU & Mininet & $\begin{array}{l}18 \text { Features - TCP Flows } \\
15 \text { Features - UDP Flows } \\
10 \text { Features - ICMP Flows }\end{array}$ & NA & $\begin{array}{c}\text { Binary Classification: } \\
\text { Normal class and DDoS } \\
\text { class. }\end{array}$ & Flow-based & [203] \\
\hline $\begin{array}{l}\text { DNN-based IDS solution } \\
\text { for SDN paradigm. }\end{array}$ & MLP & CICIDS2017 & ONOS & Mininet & 79 Features-Flow based. & $\begin{array}{c}\text { Min/Max } \\
\text { Scaler } \\
\text { Technique }\end{array}$ & $\begin{array}{c}\text { Multiclass } \\
\text { Classification: } \\
\text { Normal, DDoS, DoS, } \\
\text { PortScan, Web Attack, } \\
\text { Brute force, Bot, } \\
\text { Infiltration. }\end{array}$ & Flow-based & [191] \\
\hline $\begin{array}{l}\text { DNN-based Botnet } \\
\text { detection } \\
\text { in SDN. }\end{array}$ & MLP & HogZilla & $\begin{array}{c}\text { Not } \\
\text { Mentioned. }\end{array}$ & Not Mentioned & 22 Features-Flow based. & $\begin{array}{c}\text { Manual } \\
\text { Selection }\end{array}$ & $\begin{array}{l}\text { Binary Classification: } \\
\text { Normal and Botnet. }\end{array}$ & Flow-based & {$[193]$} \\
\hline $\begin{array}{c}\text { IDS solution for SDN } \\
\text { and evaluate different } \\
\text { ML algorithms } \\
\text { performance. }\end{array}$ & GRU-RNN & CSE-CIC-IDS2018 & $\begin{array}{c}\text { Not } \\
\text { Mentioned. }\end{array}$ & Not Mentioned & 80 Features-Flow based. & NA & $\begin{array}{c}\text { Multiclass } \\
\text { Classification: } \\
\text { Normal, DDoS, DoS, } \\
\text { Infiltration, Web Attack, } \\
\text { Brute force, Bot. }\end{array}$ & Flow-based & [194] \\
\hline $\begin{array}{c}\text { Building a modular } \\
\text { architecture to detect } \\
\text { Port Scan and DDoS } \\
\text { attack in SDN. }\end{array}$ & $\begin{array}{c}\text { LSTM } \\
\text { FUZZY } \\
\text { INFERENCE } \\
\text { LOGIC }\end{array}$ & $\begin{array}{c}\text { CIC-DDoS2019 } \\
{[212],[213]}\end{array}$ & Floodlight & Mininet & Time-stamp Features. & LSTM & $\begin{array}{c}\text { Multiclass } \\
\text { Classification: } \\
\text { Normal, DDoS, Port- } \\
\text { Scan. }\end{array}$ & $\begin{array}{l}\text { Flow-based } \\
\text { Packet- } \\
\text { based }\end{array}$ & [195] \\
\hline $\begin{array}{c}\text { Investigating the } \\
\text { performance of DL- } \\
\text { based models in DDoS } \\
\text { attack detection }\end{array}$ & $\begin{array}{l}\text { RNN } \\
\text { LSTM } \\
\text { CNN }\end{array}$ & Simulated & Floodlight & Mininet & $\begin{array}{c}7 \text { Features: } \\
\text { Src/Dst. IP address, } \\
\text { Transmission protocol, } \\
\text { Delay, throughput, Number } \\
\text { of packets, Number of hosts. }\end{array}$ & NA & $\begin{array}{l}\text { Binary Classification: } \\
\text { Normal and DDoS. }\end{array}$ & $\begin{array}{l}\text { Flow-based } \\
\text { Statistics- } \\
\text { based }\end{array}$ & [196] \\
\hline $\begin{array}{l}\text { Developing a 3-tier IDS } \\
\text { and prevention } \\
\text { framework through } \\
\text { validation of user, packet } \\
\text { and flow of the network } \\
\text { in SDN-IoT paradigm. }\end{array}$ & $\begin{array}{c}\text { CNN } \\
\text { Type-2 Fuzzy }\end{array}$ & Not mentioned. & $\begin{array}{c}\text { Not } \\
\text { Mentioned. }\end{array}$ & Not Mentioned & $\begin{array}{c}\text { 6 Features: } \\
\text { Protocol type, Transport } \\
\text { protocol, Src/Dst. IP } \\
\text { address, Src/Dst. port no. }\end{array}$ & $\mathrm{CNN}$ & $\begin{array}{c}\text { Binary Classification: } \\
\text { Normal and Malicious. }\end{array}$ & Flow-based & [197] \\
\hline $\begin{array}{l}\text { CNN-based DoS attack } \\
\text { detection. }\end{array}$ & $\begin{array}{l}\text { CNN } \\
\text { RNN }\end{array}$ & $\begin{array}{c}\text { KDD CUP } 1999 \\
\text { CSE-CIC-IDS2018 }\end{array}$ & $\begin{array}{c}\text { Not } \\
\text { Mentioned. }\end{array}$ & Not Mentioned. & $\begin{array}{c}41 \text { Features - KDD CUP } \\
1999 \\
78 \text { Features - CSE-CIC-IDS } \\
2018\end{array}$ & NA & $\begin{array}{l}\text { Binary Classification: } \\
\text { Benign \& Attack. } \\
\text { Multiclass } \\
\text { Classification: } \\
\text { Benign, Hulk, } \\
\text { SlowHTTPTest, } \\
\text { GoldenEye, Slowloris, } \\
\text { LOIC-HTTP, HOIC. }\end{array}$ & Flow-based & [198] \\
\hline $\begin{array}{l}\text { Evaluating ML methods } \\
\text { impact in flow-based } \\
\text { anomaly detection. }\end{array}$ & $\begin{array}{c}\text { RF } \\
\text { GRU-LSTM }\end{array}$ & NSL-KDD & $\begin{array}{c}\text { Not } \\
\text { Mentioned. }\end{array}$ & Not Mentioned & $\begin{array}{l}\text { Nominal, Numeric and } \\
\text { Binary Feature Set. }\end{array}$ & $\mathrm{RF}$ & $\begin{array}{c}\text { Multiclass } \\
\text { Classification: } \\
\text { Normal, DoS, R2L, } \\
\text { Probe, U2R and } \\
\text { Unknown Attacks. }\end{array}$ & Flow-based & [199] \\
\hline $\begin{array}{l}\text { Detection and prevention } \\
\text { mechanism of SSH } \\
\text { brute-force and DDoS } \\
\text { attacks in SDN. }\end{array}$ & $\begin{array}{l}\text { CNN, LSTM, } \\
\text { MLP, SAE }\end{array}$ & $\begin{array}{l}\text { Self-Generated } \\
\text { using synthetic } \\
\text { topology. }\end{array}$ & RYU & Not Mentioned & $\begin{array}{l}\text { Packet length sequence and } \\
\text { packet header information }\end{array}$ & NA & $\begin{array}{l}\text { Binary classification: } \\
\text { Normal and Attack. }\end{array}$ & Flow-based & [214] \\
\hline $\begin{array}{c}\text { Synthesized attack } \\
\text { generation using GAN in } \\
\text { SDN test the IDS against } \\
\text { those attacks. }\end{array}$ & $\begin{array}{c}\text { GAN } \\
\text { KNN } \\
\text { RF }\end{array}$ & $\begin{array}{l}\text { Self-Generated } \\
\text { using synthetic } \\
\text { topology. }\end{array}$ & $\begin{array}{c}\text { Not } \\
\text { Mentioned. }\end{array}$ & GENI [215] & Not Mentioned. & $\begin{array}{l}\text { GAN } \\
\text { RF }\end{array}$ & $\begin{array}{l}\text { Binary Classification: } \\
\text { Benign and Suspicious. }\end{array}$ & $\begin{array}{l}\text { Flow-based } \\
\text { Packet- } \\
\text { based }\end{array}$ & [216] \\
\hline $\begin{array}{l}\text { Evaluating UL based } \\
\text { AutoEncoder, and K- } \\
\text { Means algorithm to } \\
\text { recognize anomalies in } \\
\text { SDN. } \\
\end{array}$ & $\begin{array}{c}\text { AutoEncoder } \\
\text { K-Means }\end{array}$ & KDD'99 & $\begin{array}{c}\text { Not } \\
\text { Mentioned. }\end{array}$ & Not Mentioned & $\begin{array}{c}\text { All } 41 \text { Features of KDD'99 } \\
\text { dataset. }\end{array}$ & NA & $\begin{array}{l}\text { Binary classification: } \\
\text { Normal and Abnormal. }\end{array}$ & Flow-based & [204] \\
\hline $\begin{array}{l}\text { Prevent DDoS attacks } \\
\text { using a cross-plane } \\
\text { framework that uses the } \\
\text { collective intelligence of } \\
\text { both the data and control } \\
\text { plane. }\end{array}$ & $\begin{array}{l}\text { AutoEncoder } \\
\text { Softmax }\end{array}$ & $\begin{array}{l}\text { Self-generated } \\
\text { real-time network } \\
\text { traffic. }\end{array}$ & RYU & $\begin{array}{l}\text { Self-Constructed } \\
\text { with real } \\
\text { hardware setup. }\end{array}$ & $\begin{array}{l}16 \text { Features- TCP, UDP, and } \\
\text { ICMP based. }\end{array}$ & NA & $\begin{array}{c}\text { Multiclass } \\
\text { Classification: } \\
\text { Normal, UDP flood, } \\
\text { SYN flood, ICMP flood. }\end{array}$ & Flow-based & [205] \\
\hline
\end{tabular}




\begin{tabular}{|c|c|c|c|c|c|c|c|c|c|}
\hline \multicolumn{10}{|c|}{ TABLE VIII (Continued) } \\
\hline Objective & Algorithm & Dataset & Controller & Testbed & Features Selection (FS) & $\begin{array}{c}\text { FS } \\
\text { Approach }\end{array}$ & Classification Type & $\begin{array}{c}\text { IDS } \\
\text { Domain }\end{array}$ & Ref. \\
\hline $\begin{array}{l}\text { GAN-based DDoS } \\
\text { detection and prevention } \\
\text { mechanism in SDN. }\end{array}$ & $\begin{array}{l}\text { GAN } \\
\text { LSTM } \\
\text { CNN } \\
\text { MLP }\end{array}$ & CIC-DDoS 2019 & Floodlight & Mininet & $\begin{array}{c}\text { 6 Features: } \\
\text { Quantitative features: } \\
\text { bits and packets. } \\
\text { Qualitative Features: } \\
\text { Source IP \& Ports. } \\
\text { Destination IP \& Ports. }\end{array}$ & $\begin{array}{c}\text { Manual } \\
\text { Selection }\end{array}$ & $\begin{array}{c}\text { Multiclass } \\
\text { Classification: } \\
\text { Normal, NTP, DNS, } \\
\text { LDAP, MSSQL, } \\
\text { NetBIOS, SNMP, } \\
\text { SSDP, UDP, UDP-Lag, } \\
\text { WebDDoS, SYN, and } \\
\text { TFTP. }\end{array}$ & Flow-based & [206] \\
\hline $\begin{array}{c}\text { A DL-based } \\
\text { collaborative IDS for } \\
\text { VANET. }\end{array}$ & GAN & $\begin{array}{c}\text { KDD'99 } \\
\text { NSL-KDD }\end{array}$ & $\begin{array}{c}\text { Not } \\
\text { Mentioned }\end{array}$ & Not Mentioned & $\begin{array}{c}\text { All } 41 \text { Features of KDD'99 } \\
\text { dataset }\end{array}$ & NA & $\begin{array}{l}\text { Binary classification: } \\
\text { Normal and Attack. }\end{array}$ & Flow-based & [208] \\
\hline $\begin{array}{c}\text { A GRU-based DL } \\
\text { system against DDoS } \\
\text { attack in SDN. }\end{array}$ & $\begin{array}{l}\text { GRU } \\
\text { FCN }\end{array}$ & $\begin{array}{c}\text { CIC-DDoS } 2019 \\
\text { CIC-IDS-2018 }\end{array}$ & $\begin{array}{c}\text { Not } \\
\text { Mentioned }\end{array}$ & Not Mentioned & 83 Features & $\begin{array}{c}\text { Manual } \\
\text { Selection }\end{array}$ & $\begin{array}{l}\text { Binary classification: } \\
\text { Normal and Attack. }\end{array}$ & Flow-based & [217] \\
\hline $\begin{array}{l}\text { RBM-based DDoS attack } \\
\text { detection in SDN. }\end{array}$ & RBM & $\begin{array}{l}\text { Self-generated } \\
\text { using synthetic } \\
\text { topology. }\end{array}$ & POX & Mininet & Not Mentioned & NA & $\begin{array}{l}\text { Binary classification: } \\
\text { Normal and Attack. }\end{array}$ & Flow-based & [209] \\
\hline
\end{tabular}

\section{HYBRID MODELS BASED IDS IN SDN}

This section discusses the IDS schemes that have combined two or more ML and DL techniques in either supervised or unsupervised manner in various steps of training a single model, such as preprocessing, feature selection, and classification, as presented in Fig. 6 of the intrusion detection process in SDNs. By hybrid model, we mean that for feature selection module, it can use an ML or DL-based algorithm; however, the classification module can use a different ML or DL-based algorithm in a single proposed model. Those studies which have experimented with both supervised and unsupervised ML-DLbased algorithms without combining them in a single model are classified as hybrid (multiple models) in this study (see Table $\mathrm{XI}$ ). Intrusion detection performances in SDNs were individually evaluated for each supervised and unsupervised learning-based algorithm in either ML or DL field in mixed architecture.

Abdallah et al. [218] present a hybrid IDS that learn spatial and temporal features from input flow by combining the CNN and LSTM algorithms. They have employed one of the most recent SDN attack-specific dataset, InSDN [219]. The highdimensional features extracted from the $\mathrm{CNN}$ are passed into the second stage, which comprises three layers: an LSTM, a fully connected, and an output layer.

Latah and Toker [220] propose a flow statistics based five-level hybrid IDS for SDN. They have used one classifier in each layer of the model. For the $1^{\text {st }}$ level, they have applied the $\mathrm{KNN}$ method; at the $2^{\text {nd }}$ layer, an extreme learning machine was used; and for the following levels, a hierarchical extreme learning machine was used in their approach. They have adopted the NSD-KDD datasets and selected six significant features for the model.

Malik et al. [221] propose a hybrid DL framework that detects cyber threats in the SDN's control plane by leveraging the extrapolative power of LSTM and CNN. They have used the CICIDS2017 dataset for their experiment. Their proposed framework successfully detects three separate attacks: XSS, Port Scan, and Botnet with a low FAR.
A DL-based detection and prevention mechanism from SSH brute-force attack is proposed by Lee et al. in [214]. Four different DL-based models, including CNN, LSTM, MLP, and SAE, are constructed and evaluated using the proposed IDS to detect SSH brute-force attacks on SDN-enabled networks using features, such as packet length sequence and packet headers.

Encouraged by the transformer network architectures [222] text-classification and image classification task in natural language processing and computer vision, He et al. [223] and Bikmukhamedo et al. [224] have applied the transformer architecture to traffic classification and attained decent results. By combining the CNN and the transformer to apply DDoS attack detection in SDN, Wang and Li propose a hybrid DDoSTC architecture [225]. They have divided the DDosTC model into three parts: the transformer Layer, CNN layer, and dense layer. The transformer architecture provides computational proficiency and scalability, and the CNN provides robust analytical ability.

The authors in [226] create a hybrid of SVM and improved history-based IP filtering scheme and a SOM classifier to secure the network. In this scheme, the raw-data processing module gathers traffic, extracts features, and sends it to the classifier module. The ensembled classifier determines if the data is an anomaly or routine. To filter the traffic for mitigation, an enhanced history-based IP filtering scheme filtering approach is used.

To identify ICMP attacks, Nam et al. [164] suggest a hybrid ML model based on SOM and KNN. They have added four additional modules to the controller: monitor, algorithm, alert, and DDoS mitigation. During the training phase, SOM is utilized in the algorithm module to cluster traffic into intrusion and normal categories. KNN assigns a label to the network state based on the labels of the $\mathrm{k}$ nearest neighbor.

To classify an abnormal flow in the SDN-based Smart Grid communication network, Ding et al. suggest a HYBRID-CNN approach [227]. The HYBRID-CNN, in particular, employs a DNN to efficiently learn global features from one-dimensional (1D) data and a CNN to generalize local features from twodimensional (2D) data. The HYBRID-CNN uses dual-channel data input to extract useful features from 1D and 2D flow data. 
It fuses essential features using the self-attention process and ultimately detects using an FCN.

Elsayed et al. proposed a new hybrid DL-approach based on CNN and SD-Reg, a new regularizer technique [228]. The model incorporates the $\mathrm{CNN}$ architecture as well as machine learning methods (SVM, KNN, and RF). CNN is used to extract the deeper representations of the data features, while ML methods are used to accomplish the classification task. The new regularization technique avoids the risk of overfitting the model.

A hybrid two-module DL-based model is presented by Garg et al. [229] detects anomaly for abnormal detection of flow in the sector of social multimedia. For anomaly detection module, it uses enhanced RBM and Gradient Descent based SVM to identify anomalous activities. Their module contains two parts, namely selection of features and classification of anomaly. In addition to the anomaly recognition module, they have also suggested a method that safeguards the frequently carriage of the multimedia content over the SDN paradigm.

Khan and Akhunzada [230] propose a hybrid deep learning model for the detection of malwares in IoMT. The model is composed of CNN and LSTM. The CNN models extract the local features and pass the output to the LSTM model to acquire more independent features to train the hybrid model.

By configuring the sampling rate for each switch, Kim et al. [184] propose a deep reinforcement learning-based traffic monitoring system in an SDN environment. They utilized a deep deterministic policy gradient-based method to manage MDPs with continuous action spaces to address intrusion utilizing realtime traffic analyzers and monitoring findings dynamically. The suggested system learns a sampling resource distribution strategy based on selected traffic inspection findings received from various traffic analyzers under the uncertainty of flow distribution.

Jiaqi et al. [231] propose an IDS solution for 5G enabled SDN paradigm. For feature selection using sub features of the dataset, it utilizes RF and combines K-Means with the AdaBoost classifier to classify flows. A comparative analysis of balanced and imbalanced data by evaluating cross validation technique results was also performed.

AlEroud and Karabatis [216] propose a GAN-based method that generates synthetic attacks in SDN. Their method collects flow entries sent back and forth between the controller and OF switches. Similarity analysis of those OFs flow was done against the non-OFs flow. They have hypothesized that the sampling flow entries of OFs and testing those using appropriate IDS can be used to determine threats on SDN. For data generation, they have used two samples. The first sample was collected from a dataset of one hour of malicious traffic from a DDoS attack. Only IP packets are used in the second sample. Both OF and non-OF data were used to generate attacks. The GAN structure in their study consists of a Generator network which creates an agitated form of attack examples, a Discriminator network which provides feedback to the generator for any modification of weights to create sufficient samples to dodge the IDS, and an intrusion detector that manages both OF and Non-OF traffic. The generated attacks were used against IDS built with KNN and RF. The detection performance of the IDS drastically reduced when tested against GAN-generated attacks.

Combining three supervised and one unsupervised ML algorithm, namely KNN, NB, SVM, and SOM, Deepa et al. [232] present an ensemble model for recognizing DDoS attacks in the SDN controller. By creating a Mininet virtual setup and a POX controller, they have created a virtual network setup, then applied the CAIDA 2016 dataset into the network consisting of TCP, ICMP, and UDP packets. A time difference of $0.004 \mathrm{sec}$ between the source and the destination is chosen as the threshold value to distinguish between attack and regular traffic. In their system, the SL-based models are passed through traffic, and the type of traffic is identified. If a novel type of attack is identified, the very same supervised learning systems is carried through the unsupervised configuration to recognize traffic, and the connection is then terminated.

Javed et al. propose a DL-based hybrid method by incorporating the DNN, LSTM, and GRU algorithms [233] to detect cyber-attacks in the IoT environment. In their approach, SDN's programmable control plane accommodates the hybrid intrusion detection model. The proposed model consists of several layers. DNN-LSTM is made up of two layers, having 500 and 300 neurons, respectively. They've also added a DNN-GRU layer of 200 neurons. In the output layer, Softmax is employed. The model achieved an efficient and accurate IDS solution by consuming a very little testing time of $9.33 \mathrm{~ms}$.

Tan et al. [234] suggest a hybrid DDoS detection trigger mechanism by merging K-Means and KNN on the SDN data plane. This technique counts the rate at which packet_in messages are sent on switches by utilizing the CPU resources of the switches. It notifies the controller to identify the abnormality when it detects the possibility of a DDoS attack, allowing the controller to respond quickly to the detection trigger mechanism. It comprises a training data processing module based on the $\mathrm{K}$ Means algorithm and a traffic detection module based on the KNN algorithm. The K-Means technique is only used to categorize related instances into distinct categories during the training phase. The measured instance is first normalized in the detection phase. The distances between that instance and the cluster centers are then determined. Finally, the measured instance is classified as normal or abnormal based on the labels of the $\mathrm{k}$ points nearest to it [16].

By combining entropy and C4.5 algorithm to detect DDoS flooding attack in SDN, Sudar and Deepalakshmi [235] have proposed a two-level IDS. A suspicious detection module and a C4.5 classification module make up the proposed system. In level one, an entropy-based approach is suggested to identify DDoS flooding attacks early on by temporarily interrupting a 
specific flow. A C4.5 approach based on ML is presented in level two to identify the attack by assessing additional attributes and sending a permanent notification to drop the packets. To identify DDoS attacks, the suspicious detection module analyzes the entropy of the IP addresses of the source to a threshold. The retrieved characteristics are evaluated, and a DDoS attack classification is done in the $\mathrm{C} 4.5$ classification module.

Table IX presents a tabular summary of the aforementioned reviewed articles that depicts the objective of the study, adopted Hybrid (ML+DL) algorithms for building IDS solution, used dataset for the task, core feature selection, chosen SDN controller, and the attack classification type, corresponding IDS domain along with their simulated testbed.

In the next section, we discuss those studies which have used ensemble-based approaches to develop IDS solutions specific to SDN.

\section{ENSEMBLE MODELS BASED IDS IN SDN}

The ensemble represents a group of independently trained classifiers, such as neural networks or DTs as shown in Fig. 16. The results are combined while categorizing new data points or instances to improve overall performance [236]. Models that belong to the ensemble, known as ensemble members, might be similar types or from various types and might not be trained on similar training data. Although ensemble learning is a very efficient technique, it does have some trade-offs. If an ensemble has one excellent performing member method and some other member methods do not give many benefits, or the ensemble cannot make effective use of their participation, this may lead to poor performance or unexpected outcome.

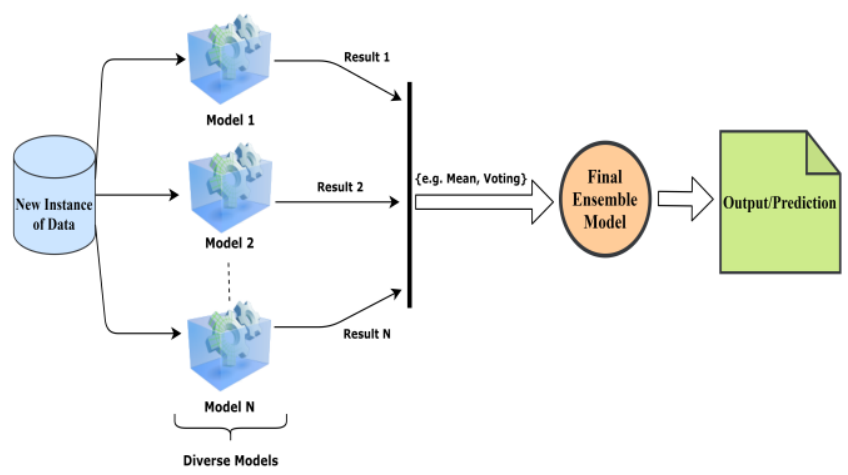

Fig. 16. A generic architecture of an ensemble learning-based model.

\section{Random Forest (RF)}

$\mathrm{RF}$ is another very useful algorithm that is being used for regression and classification. By constructing multiple DT's and combining them, RF is providing a more efficient and comprehensive prediction, as shown in Fig. 17. RF is a mixture of tree predictors, in which every tree depending on the values of the arbitrary vectors, is sampled separately with almost the similar dissemination across all the trees in the forest [237]. RF make the data more general in a better way. This randomized set of features make the RF much more precise than the DT algorithm. RF is suitable when we have a larger dataset, and interpretability is not our core concern.

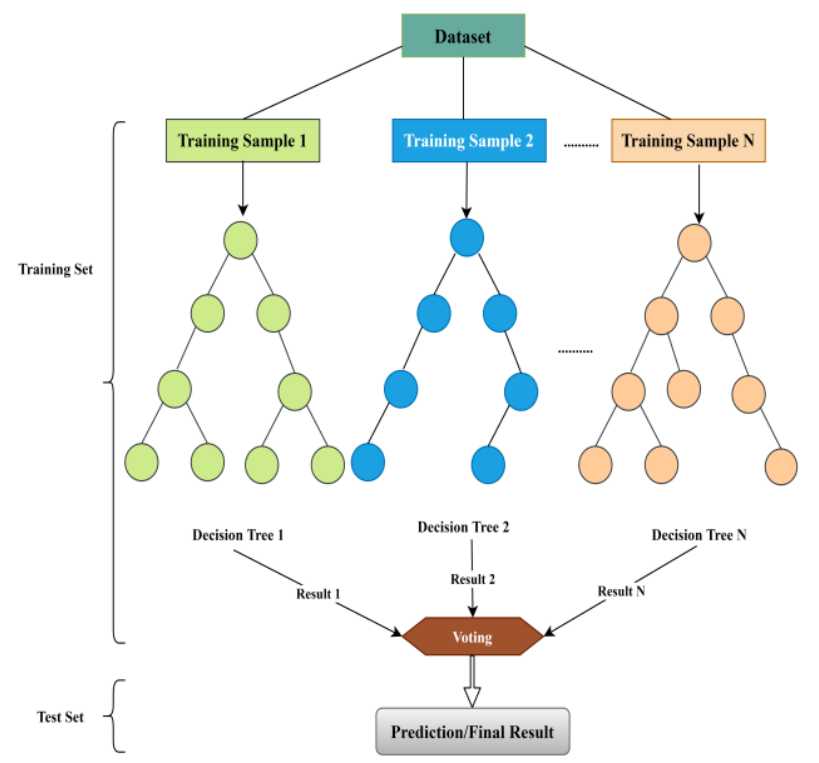

Fig. 17. A random forest (RF) classifier based on multiple DT's.

Most popular ensemble methods include RF, boosting, bagging, cascading and stacking. Boosting is a useful tool that learns flaws from previous models to improve classification efficiency [238]. Bagging improves the performance of the prediction by using DT's that decreases variance to a substantial measure. Bagging is categorized into two kinds: bootstrapping and aggregation. Stacking functions through enabling a training algorithm to combine a number of related learning algorithms result.

Demirpolat et al. [239] propose a novel SDN-specific intrusion dataset in their study. They have used Few Shots Learning Classifier (FSLC), weighted average ensemble, and SVM models to detect intrusion in SDN by evaluating performance in two publicly available datasets UNSW-NB15, Bot-IoT, and one proposed SDN Dataset. They have also highlighted how traditional intrusion datasets cannot capture full information regarding SDN architecture.

Swami et al. [240] propose two ensemble models named Voting-CMN combining CART, MLP, and NB algorithm and Voting-RKM combining RF, KNN, and MLP algorithm to identify DDoS attacks in SDN architecture. They have evaluated those models' performance along with each model used in the ensemble using the UNSW-NB15, CICIDS2017, and NSL-KDD datasets. Voting-CMN performed better than individual CART, MLP, and NB models with an F1-Score of $99.28 \%$ and an accuracy of $89.29 \%$ in the UNSW-NB15 dataset. Voting-RKM achieved an accuracy of $97.77 \%$ and F1-Score of $96.36 \%$ on the CICIDS2017 dataset, which is better than individual models comprising that architecture. However, the False Alarm Rate of Voting-CMN performed poorly with a value of $20.2 \%$. 


\begin{tabular}{|c|c|c|c|c|c|c|c|c|c|}
\hline \multicolumn{10}{|c|}{$\begin{array}{c}\text { TABLE IX } \\
\text { RY OF THE REVIEWED HYBRID MODELS TO DETECT INTRUSION IN SDN }\end{array}$} \\
\hline Objective & Algorithm & Dataset & Controller & Testbed & Features Selection (FS) & $\begin{array}{c}\text { FS } \\
\text { Approach }\end{array}$ & Classification Type & $\begin{array}{c}\text { IDS } \\
\text { Domain }\end{array}$ & Ref. \\
\hline $\begin{array}{l}\text { SOM-based methods in } \\
\text { DDoS flooding attack } \\
\text { detection using SDN. }\end{array}$ & $\begin{array}{l}\text { SOM } \\
\text { KNN }\end{array}$ & $\begin{array}{c}\text { Caida DDoS } \\
\text { Attack } 2007[241]\end{array}$ & POX & Not Mentioned & $\begin{array}{l}5 \text { Features: Entropy of } \\
\text { source IP, entropy of source } \\
\text { port, entropy of destination } \\
\text { port, entropy of packet } \\
\text { protocol, total no. of } \\
\text { packets. }\end{array}$ & $\begin{array}{c}\text { Manual } \\
\text { Selection }\end{array}$ & $\begin{array}{l}\text { Binary Classification: } \\
\text { Normal and Attack. }\end{array}$ & Flow-based & {$[164]$} \\
\hline $\begin{array}{l}\text { Flow-based 5-level } \\
\text { hybrid IDS in SDN. }\end{array}$ & KNN & NSL-KDD & POX [30] & Not Mentioned & $\begin{array}{c}5 \text { Features: Duration, } \\
\text { Protocol type, Src. bytes, } \\
\text { Dst. bytes, and Server count. }\end{array}$ & $\begin{array}{c}\text { Manual } \\
\text { Selection }\end{array}$ & $\begin{array}{c}\text { Multiclass } \\
\text { Classification: } \\
\text { Normal, DoS, R2L, } \\
\text { Probe, U2R and } \\
\text { Unknown Attacks. }\end{array}$ & Flow-based & {$[220]$} \\
\hline $\begin{array}{l}\text { Developing a hybrid DL } \\
\text { based Reconnaissance } \\
\text { and Surveillance } \\
\text { Recognition method on } \\
\text { the control plane of SDN. }\end{array}$ & $\begin{array}{l}\text { LSTM } \\
\text { CNN }\end{array}$ & CICIDS2017 & $\begin{array}{c}\text { Not } \\
\text { Mentioned }\end{array}$ & Not Mentioned & 83 Features-Flow based. & NA & $\begin{array}{c}\text { Multiclass } \\
\text { Classification: } \\
\text { Benign, Botnet, Port } \\
\text { Scan, Cross Site } \\
\text { Scripting, Brute force. }\end{array}$ & Flow-based & {$[221]$} \\
\hline $\begin{array}{l}\text { A transformer-based } \\
\text { hybrid IDS mechanism in } \\
\text { SDN. }\end{array}$ & $\begin{array}{l}\text { Transformer } \\
\text { CNN } \\
\text { FCN }\end{array}$ & CICDDoS2019 & $\begin{array}{c}\text { Not } \\
\text { Mentioned }\end{array}$ & Not Mentioned & 76- Flow features & NA & $\begin{array}{l}\text { Binary classification: } \\
\text { Normal and DDoS. }\end{array}$ & Flow-based & [225] \\
\hline $\begin{array}{c}\text { Effective Defense } \\
\text { mechanism to detect } \\
\text { DDoS attack in SDN- } \\
\text { based cloud. }\end{array}$ & $\begin{array}{l}\text { SOM, SVM, } \\
\text { and History- } \\
\text { based IP } \\
\text { filtering. }\end{array}$ & $\begin{array}{l}\text { CAIDA and } \\
\text { Synthetic data } \\
\text { using BoNeSi. }\end{array}$ & $\begin{array}{c}\text { Not } \\
\text { Mentioned }\end{array}$ & Self-Constructed & $\begin{array}{c}\text { 10 Features: } \\
\text { Flow duration, Packet } \\
\text { number, Byte number, } \\
\text { Protocol, Active time, } \\
\text { Priority, Flag, Flow number } \\
\text { and Transferred packet per } \\
\text { flow. }\end{array}$ & $\begin{array}{c}\text { Not } \\
\text { Mentioned }\end{array}$ & $\begin{array}{l}\text { Binary classification: } \\
\text { Normal and DDoS. }\end{array}$ & $\begin{array}{c}\text { Flow-based } \\
\text { Statistics- } \\
\text { based }\end{array}$ & {$[226]$} \\
\hline $\begin{array}{l}\text { Deep RL-based traffic } \\
\text { sampling for several } \\
\text { traffic analyzers in SDN. }\end{array}$ & $\begin{array}{l}\text { DDPG, } \\
\text { MDP }\end{array}$ & $\begin{array}{l}\text { Generated traffic } \\
\text { using Iperf, and } \\
\text { Hping3 tools. }\end{array}$ & OpenDaylight & Self-Constructed & Not Mentioned. & $\begin{array}{c}\text { Not } \\
\text { Mentioned }\end{array}$ & $\begin{array}{l}\text { Binary Classification: } \\
\text { Normal and Malicious. }\end{array}$ & $\begin{array}{l}\text { Flow-based } \\
\text { Packet-based }\end{array}$ & [184] \\
\hline $\begin{array}{c}\text { A hybrid-CNN based } \\
\text { abnormal flow detection } \\
\text { model in the SDN-Based } \\
\text { Smart Grid. }\end{array}$ & $\begin{array}{l}\mathrm{CNN} \\
\mathrm{FCN}\end{array}$ & $\begin{array}{l}\text { UNSW-NB15 } \\
\text { KDD'99 }\end{array}$ & $\begin{array}{c}\text { Not } \\
\text { Mentioned }\end{array}$ & Not Mentioned & 42 - Flow features & N/A & $\begin{array}{c}\text { Multiclass } \\
\text { Classification: } \\
\text { Normal, DoS, R2L, } \\
\text { Probe, U2R. }\end{array}$ & Flow-based & [227] \\
\hline $\begin{array}{l}\text { A novel hybrid model for } \\
\text { ids in SDN-based on } \\
\text { CNN and a new } \\
\text { regularization method. }\end{array}$ & $\begin{array}{c}\text { CNN } \\
\text { KNN } \\
\text { RF } \\
\text { SVM }\end{array}$ & InSDN & $\begin{array}{c}\text { Not } \\
\text { Mentioned }\end{array}$ & Not Mentioned & $\begin{array}{c}48 \text { - SDN specific flow } \\
\text { features }\end{array}$ & NA & $\begin{array}{c}\text { Both Multiclass and } \\
\text { Binary classification. } \\
\text { Binary: Normal and } \\
\text { Attack. } \\
\text { Multiclass: Normal, } \\
\text { U2R, Web, Probe, } \\
\text { Password guessing, DoS } \\
\text { (HULK, TorshHammer, } \\
\text { HTTP-Flood), DDoS, } \\
\text { and Botnet. }\end{array}$ & Flow-based & [228] \\
\hline $\begin{array}{c}\text { Suspicious Flow } \\
\text { Recognition in SDN. }\end{array}$ & $\begin{array}{c}\text { RBM } \\
\text { Gradient } \\
\text { Descent based } \\
\text { SVM }\end{array}$ & $\begin{array}{l}\text { Real-time data } \\
\text { traffic and KDD99 } \\
\text { benchmark dataset. }\end{array}$ & $\begin{array}{c}\text { Not } \\
\text { Mentioned }\end{array}$ & Not Mentioned & $\begin{array}{c}\text { Real-time: } 35 \text { features. } 10 \\
\text { basic features, } 8 \text { content- } \\
\text { based and } 6 \text { host-based } \\
\text { features. } \\
\text { KDD99: } 41 \text { features. }\end{array}$ & RBM & $\begin{array}{c}\text { Multiclass } \\
\text { Classification: } \\
\text { Normal, DoS, R2L, } \\
\text { Probe, U2R and } \\
\text { Unknown Attacks. }\end{array}$ & Flow-based & [229] \\
\hline $\begin{array}{l}\text { A hybrid DL-based SDN- } \\
\text { enabled malware } \\
\text { detection framework for } \\
\text { IoMT. }\end{array}$ & $\begin{array}{l}\text { CNN } \\
\text { LSTM }\end{array}$ & IoT-23 [242] & $\begin{array}{c}\text { Floodlight, } \\
\text { POX, and } \\
\text { Open daylight }\end{array}$ & Not Mentioned & $24-$ Flow features & NA & $\begin{array}{l}\text { Binary classification: } \\
\text { Normal and Malware. }\end{array}$ & Flow-based & [230] \\
\hline $\begin{array}{c}\text { IDS solution for 5G } \\
\text { enabled SDN paradigm. }\end{array}$ & $\begin{array}{c}\text { RF, } \\
\text { K-Means, } \\
\text { AdaBoost }\end{array}$ & $\begin{array}{c}\text { KDD99, } \\
\text { NSL-KDD }\end{array}$ & $\begin{array}{c}\text { Not } \\
\text { Mentioned }\end{array}$ & Not Mentioned & $\begin{array}{c}\text { KDD99 - } 41 \text { Features } \\
\text { NSL-KDD - } 23 \text { Features }\end{array}$ & $\mathrm{RF}$ & $\begin{array}{c}\text { Multiclass } \\
\text { Classification: } \\
\text { Normal, DoS, R2L, } \\
\text { Probe, U2R. }\end{array}$ & Flow-based & [231] \\
\hline $\begin{array}{l}\text { DL-based hybrid IDS } \\
\text { solution in SDN-IoT } \\
\text { environment. }\end{array}$ & $\begin{array}{c}\text { DNN-LSTM } \\
+ \\
\text { DNN-GRU }\end{array}$ & CIC-DDoS-2019 & $\begin{array}{c}\text { Not } \\
\text { Mentioned }\end{array}$ & Not Mentioned & 80 Flow features. & N/A & $\begin{array}{c}\text { Multiclass } \\
\text { Classification: } \\
\text { Normal, SYN, PortMap, } \\
\text { DrDoS_MSSQL, } \\
\text { DrDoS_SSDP, } \\
\text { WebDDoS, WebDDoS, } \\
\text { DR DoS, DrDoS_UDP, } \\
\text { UDP-Lag. }\end{array}$ & Flow-based & [233] \\
\hline $\begin{array}{c}\text { A hybrid trigger } \\
\text { mechanism-based DDoS } \\
\text { detection on SDN data } \\
\text { plane. }\end{array}$ & $\begin{array}{c}\text { KNN } \\
+ \\
\text { K-Means }\end{array}$ & NSL-KDD & ONOS & Mininet & 41 - Flow Features & K-Means & $\begin{array}{l}\text { Binary Classification: } \\
\text { Normal and DDoS. }\end{array}$ & Flow-based & {$[234]$} \\
\hline $\begin{array}{l}\text { A hybrid entropy and } \\
\text { C4.5-based two-level IDS } \\
\text { solution for SDN. }\end{array}$ & $\begin{array}{c}\text { Entropy } \\
+ \\
\text { C4.5 }\end{array}$ & $\begin{array}{l}\text { Self-Generated } \\
\text { using synthetic } \\
\text { tool. }\end{array}$ & POX & Mininet & $\begin{array}{c}\text { 6 Features: Average } \\
\text { number of Packets per Flow, } \\
\text { Average number of Bytes } \\
\text { per Flow, Average of } \\
\text { Duration per Flow, Pair } \\
\text { Flows Percentage, Growth } \\
\text { of Single Flows, and Growth } \\
\text { of Different Ports. }\end{array}$ & $\mathrm{C} 4.5$ & $\begin{array}{l}\text { Binary Classification: } \\
\text { Normal and Attack. }\end{array}$ & $\begin{array}{c}\text { Flow-based } \\
\text { Statistics- } \\
\text { based }\end{array}$ & {$[235]$} \\
\hline
\end{tabular}


Shahzeb et al. [243] present a DL-based ensemble model for classifying DDoS attacks using the ISCX dataset. They provided an ensemble solution by implementing two CNN models. The authors also claimed that the proposed models are scalable and cost-effective in terms of CPU utilization. The experiment result shows that the proposed model achieved $99.48 \%$ accuracy to identify DDoS attacks.

Zwane et al. [244] evaluate different ensemble methods to detect abnormal flows in SDN and compare their performance with single classifier models. The experiment is conducted using a flow-based CIDDS-001 dataset. They used the DT, NB, and SVM as a single classifier. On the other hand, Bagging, Adaboost, RF, and Majority Voting are used as ensemble methods. The accuracy of the ensemble methods is slightly greater than the accuracy of the individual SL-based nonensemble methods. The NB model achieved $69.56 \%$ accuracy, whereas the bagging version of NB model achieves $70.74 \%$ accuracy.

Zwane et al. [245] propose an ML-based IDS for Tactical Ad hoc Mobile Network (TMANET) that resembles the concept of SDN. Their proposed model consists of two SDN controllers: local and global. The global controller manages the overall network and different ML methods. SVM, DT, Bagging, Adaboost, and RF are used as classification methods. On the other hand, the local controller deals with D2D connection, roles of nodes, embedded sampling agent, etc. A flow-based CIDDS001 dataset is used to train and test the ML models. Ensemble method Adaboost performs best among other models with $90.3 \%$ accuracy according to the experiment result.

Yang et al. [246] present a DL-based UL-based model named Griffin, to detect SDN anomaly. Griffin is a packet-based anomaly recognition system that can revise the model dynamically to adapt to the recent cyberattacks. Griffin architecture has four parts, which are deployed in SDN, utilizing an ensemble of AutoEncoders. These four parts are packet capture module, feature extraction module, feature mapper module, and anomaly detector, respectively. Each packet with nine characteristics is accumulated in packet capture parts. Feature extractor generates statistically significant features. Feature mapper subdivides each feature into several instances for increasing memory efficiency. The anomaly detector is placed in the SDN control plane, where the ensemble of AutoEncoders is responsible for filtering out the anomalous packet. The model's performance was tested with an open data set contributed by Yisroel Mirsky [247]. The dataset contains packets with four different attack types: DoS, MITM, Recon, and Bot Malware.

To detect the DDoS attacks in the SDN cloud, a DT-based ensemble method is proposed by Cheng et al. [248]. The authors claimed that their proposed eXtreme Gradient Boosting (XGBoost) models exhibit higher accuracy and provide highspeed anomaly detection. The experiment is executed in a simulated SDN topology using Mininet, and Hyenae is used as attack-generation tools. The XGBoost model is trained and tested using the KDD'99 dataset by selecting nine significant features out of 41 features from the dataset. The proposed XGBoost model achieved $98.53 \%$ accuracy.

Sen et al. [249] propose a DDoS attack recognition model by creating a Mininet testbed for SDN. They have used the sFlowRT network analyzer for traffic monitoring. Their model achieved 93\% detection accuracy using the AdaBoost classifier.

Van et al. [250] present an anomaly detection framework based on the NetFPGA-10G board, including an anomaly-based IDS based on the J48 algorithm. The suggested framework is separated into three sections: Input, Processing, and Output. The data and control input ports on the Input unit are used to receive incoming packets. The processing unit handles incoming packet processing, OpenFlow processing, security processing, and outbound packet processing. After being processed by the processing components, the packet is transferred to an output queue. The queue delivers this packet to a relevant data output port based on OpenFlow protocol routing information. Initially, they conducted five J48 DT analyses on the KDD CUP 1999 dataset. The Bagging method was then used to generate the outcomes of five different DTs, and electoral rules were used to choose the final result.

To predict attacks and effectively reduce the packet drop ratio, Alamri and Thayananthan [251] propose an adaptive bandwidth control procedure. Their framework consists of three phases: monitoring, bandwidth control, and attack detection and alleviation phase. They used a bandwidth management algorithm combined with an adaptive threshold to reprimand flows that exceeded the threshold by using a punishment mechanism that confines the bandwidth by half for flow exceeding the threshold, preventing bandwidth exhaustion and ensuring smooth operation of the network even during an attack. They used the XGBoost algorithm to analyze and identify traffic as normal or DDoS using a trigger-based identification and classification strategy. They have tested their model in three datasets: CICDDoS2019, CAIDA-DDoS and NSL-KDD. They have performed both binary and multiclass classification. The binary module classifies normal and DDoS attacks. The multiclass classifier classifies Normal, DNS, LDAP, MSSQL, SYN, UDP, NetBIOS, TFTP, NTP, UDP-lag, and SSDP attack from CICDDoS2019 dataset and Normal, Apache2, Back, Land, Mailbomb, Smurf, Teardrop, Pod, Neptune, and Processtable attack from NSL-KDD dataset.

Alzahrani and Alenazi [252] explore the tree-based DT, RF, and XGBoost algorithm to analyze their performance in detecting attacks in SDN using only five features of the NSLKDD dataset. XGBoost performs better with a higher accuracy rate of $96.55 \%$ in the binary classification problem and similar performances in the multiclass problem.

Table $\mathrm{X}$ presents a tabular summary of the reviewed literature based on utilizing ensemble models for developing IDS solution for SDN infrastructure. 


\begin{tabular}{|c|c|c|c|c|c|c|c|c|c|}
\hline \multicolumn{10}{|c|}{$\begin{array}{c}\text { TABLE X } \\
\text { MBLE LEARNING-BASED MODELS TO DETECT INTRUSION IN SDN }\end{array}$} \\
\hline Objective & Algorithm & Dataset & Controller & Testbed & Features Selection (FS) & FS Approach & Classification Type & $\begin{array}{c}\text { IDS } \\
\text { Domain }\end{array}$ & Ref. \\
\hline $\begin{array}{c}\text { Intrusion Detection in } \\
\text { SDN using Few Shots } \\
\text { ensemble learning, and } \\
\text { proposing a novel SDN } \\
\text { specific dataset. }\end{array}$ & $\begin{array}{l}\text { FSLC, } \\
\text { SVM }\end{array}$ & $\begin{array}{l}\text { Bot-IoT, UNSW- } \\
\text { NB15, and } \\
\text { proposed SDN } \\
\text { dataset. }\end{array}$ & ONOS & Mininet & $\begin{array}{l}\text { UNSW-NB15-16 Features. } \\
\text { Bot-IoT - 16 Features. } \\
\text { SDN dataset - 10 Features } \\
\text { (Duration, Mean, Standard } \\
\text { Deviation, Summation, } \\
\text { Minimum, Maximum of } \\
\text { records duration, packet } \\
\text { count from src. to dst. and } \\
\text { vice versa, byte count from } \\
\text { src. to dst. and vice versa ). }\end{array}$ & $\begin{array}{c}\text { Based on } \\
\text { Flow entry } \\
\text { statistics. }\end{array}$ & $\begin{array}{c}\text { Multiclass } \\
\text { Classification: } \\
\text { DDoS, DoS, Port } \\
\text { Scanning, Fuzzing, } \\
\text { Exploits, Fuzzers, } \\
\text { Reconnaissance, } \\
\text { Generic, } \\
\text { Os_Fingerprint, OS_and } \\
\text { Service_Detection } \\
\text { Service_scan, Theft. }\end{array}$ & $\begin{array}{l}\text { Flow-based } \\
\text { Statistics- } \\
\text { based }\end{array}$ & [239] \\
\hline $\begin{array}{l}\text { Developing voting- } \\
\text { based DDoS attack } \\
\text { detection framework } \\
\text { against SDN. }\end{array}$ & $\begin{array}{c}\text { CART, MLP, } \\
\text { NB, RF, } \\
\text { KNN, NB }\end{array}$ & $\begin{array}{l}\text { UNSW-NB15, } \\
\text { CICIDS2017, and } \\
\text { NSL-KDD }\end{array}$ & $\begin{array}{c}\text { Not } \\
\text { Mentioned }\end{array}$ & Not Mentioned & $\begin{array}{l}\text { UNSW-NB15-49 Features. } \\
\text { CICIDS2017-78 Features. } \\
\text { NSL-KDD-41 Features. }\end{array}$ & $\begin{array}{c}\text { Manual } \\
\text { Selection }\end{array}$ & $\begin{array}{l}\text { Binary Classification: } \\
\text { Normal and DDoS. }\end{array}$ & Flow-based & [240] \\
\hline $\begin{array}{c}\text { DDoS attack detection } \\
\text { performance analysis } \\
\text { between individual ML } \\
\text { algorithm and ensemble } \\
\text { learning. }\end{array}$ & $\begin{array}{l}\text { SVM, } \\
\text { KNN, } \\
\text { NB, } \\
\text { SOM }\end{array}$ & CAIDA 2016 & POX & Mininet & $\begin{array}{l}\text { Not Mentioned } \\
\text { Specifically. }\end{array}$ & $\begin{array}{c}\text { Manual } \\
\text { Extraction of } \\
\text { qualitative } \\
\text { and } \\
\text { quantitative } \\
\text { features. } \\
\end{array}$ & $\begin{array}{l}\text { Binary Classification: } \\
\text { Normal and DDoS. }\end{array}$ & Flow-based & {$[232]$} \\
\hline $\begin{array}{c}\text { Detection of DDoS } \\
\text { attack using CNN in } \\
\text { SDN } \\
\end{array}$ & $\mathrm{CNN}$ & ISCX 2012 & $\begin{array}{c}\text { Not } \\
\text { Mentioned }\end{array}$ & Not Mentioned & $\begin{array}{l}4 \text { Features-Packet length, } \\
\text { Avg. packet size, duration, } \\
\text { and IAT. }\end{array}$ & $\begin{array}{c}\text { Z-score } \\
\text { normalization. }\end{array}$ & $\begin{array}{l}\text { Binary Classification: } \\
\text { Normal and DDoS }\end{array}$ & Flow-based & {$[243]$} \\
\hline $\begin{array}{c}\text { Compare the } \\
\text { performance of different } \\
\text { ensemble methods with } \\
\text { single model classifiers. }\end{array}$ & $\begin{array}{l}\text { SVC, NB, } \\
\text { DT, RF, } \\
\text { Bagging, } \\
\text { Adaboost, } \\
\text { Majority } \\
\text { Voting. }\end{array}$ & CIDDS-001 & $\begin{array}{c}\text { Not } \\
\text { Mentioned }\end{array}$ & Not Mentioned & 14 Features-Flow based. & $\begin{array}{c}\text { Not } \\
\text { Mentioned }\end{array}$ & $\begin{array}{c}\text { Multiclass } \\
\text { Classification: } \\
\text { 5 Classes (normal, } \\
\text { attacker, victim, } \\
\text { suspicious, unknown) }\end{array}$ & Flow-based & {$[244]$} \\
\hline $\begin{array}{c}\text { Design a flow-based } \\
\text { anomaly classifier for } \\
\text { Tactical Mobile Adhoc } \\
\text { Network (TMANET) } \\
\text { incorporating with ML } \\
\text { and SDN. }\end{array}$ & $\begin{array}{c}\text { SVM, } \\
\text { DT, } \\
\text { Bagging, } \\
\text { Adaboost, } \\
\text { RF }\end{array}$ & CIDDS-001 & $\begin{array}{c}\text { Not } \\
\text { Mentioned }\end{array}$ & Not Mentioned & 14 Features-Flow based. & $\begin{array}{l}\text { Manual } \\
\text { Selection }\end{array}$ & $\begin{array}{c}\text { Multiclass } \\
\text { Classification: } \\
5 \text { Classes (normal, } \\
\text { attacker, victim, } \\
\text { suspicious, unknown) }\end{array}$ & Flow-based & {$[245]$} \\
\hline $\begin{array}{c}\text { Develop an } \\
\text { unsupervised DL-based } \\
\text { model to intensify the } \\
\text { accuracy through } \\
\text { dynamically train the } \\
\text { model. }\end{array}$ & Autoencoder & $\begin{array}{l}\text { Open-Source } \\
\text { Dataset }\end{array}$ & $\begin{array}{c}\text { Not } \\
\text { Mentioned }\end{array}$ & Mininet & $\begin{array}{l}23 \text { Features-based on } \\
\text { packets size, jitter, } \\
\text { magnitude, and count. }\end{array}$ & $\mathrm{RF}$ & Not Mentioned. & Flow-based & [246] \\
\hline $\begin{array}{l}\text { Detection of DDoS } \\
\text { attack using DT based } \\
\text { ensemble method }\end{array}$ & XGBoost & KDD'99 & POX & Mininet & $\begin{array}{c}9 \text { Features: } \\
\text { Service, source bytes, } \\
\text { packet count, service count, } \\
\text { dst. host count, dst. host } \\
\text { service count, dst. host } \\
\text { service rate, dst. host } \\
\text { service error rate } \\
\end{array}$ & $\begin{array}{c}\text { Not } \\
\text { Mentioned }\end{array}$ & $\begin{array}{c}\text { Multiclass } \\
\text { Classification: } \\
\text { Normal, DoS, R2L, } \\
\text { Probe, U2R. }\end{array}$ & Flow-based & [248] \\
\hline $\begin{array}{l}\text { DDoS attack detection } \\
\text { through various ML } \\
\text { algorithms. }\end{array}$ & $\begin{array}{c}\text { AdaBoost, } \\
\text { Bayes Net, } \\
\text { NB, SVM, } \\
\text { MLP, J48, RF }\end{array}$ & $\begin{array}{c}\text { Self-collected } \\
\text { dataset generated } \\
\text { from synthetic } \\
\text { topology. }\end{array}$ & $\begin{array}{c}\text { Not } \\
\text { Mentioned }\end{array}$ & Mininet & $\begin{array}{l}9 \text { Features: Service, TTL, } \\
\text { Header Length, Flags, } \\
\text { Protocols, Data Bytes, } \\
\text { Land, Epoch Time and } \\
\text { Reply Response Time }\end{array}$ & $\begin{array}{c}\text { Not } \\
\text { Mentioned }\end{array}$ & $\begin{array}{l}\text { Binary Classification: } \\
\text { Normal and DDoS. }\end{array}$ & Flow-based & [249] \\
\hline $\begin{array}{l}\text { Implementing a 3-phase } \\
\text { IDS solution in SDN to } \\
\text { detect DDoS attack } \\
\text { along with a bandwidth } \\
\text { control method utilizing } \\
\text { a threshold function to } \\
\text { penalize the corrupted } \\
\text { flow. }\end{array}$ & XGBoost & $\begin{array}{l}\text { CIC-DDoS-2019, } \\
\text { CAIDA-DDoS, } \\
\text { NSL-KDD. }\end{array}$ & RYU & Mininet & $\begin{array}{c}\text { CICDDoS2019 - } 20 \\
\text { Features } \\
\text { NSL-KDD - 24 Features } \\
\text { CAIDA - } 7 \text { Features }\end{array}$ & $\begin{array}{l}\text { Information } \\
\text { Gain }\end{array}$ & $\begin{array}{c}\text { Both Binary } \\
\text { Classification and } \\
\text { Multiclass } \\
\text { Classification: } \\
\text { Normal, DoS, R2L, } \\
\text { Probe, U2R. }\end{array}$ & Flow-based & {$[251]$} \\
\hline $\begin{array}{c}\text { Evaluating the attack } \\
\text { detection performance } \\
\text { of the Tree-based DT, } \\
\text { RF and XGBoost } \\
\text { classifier. }\end{array}$ & $\begin{array}{c}\text { DT, RF, } \\
\text { XGBoost }\end{array}$ & NSL-KDD & $\begin{array}{c}\text { Not } \\
\text { Mentioned }\end{array}$ & Not Mentioned & $\begin{array}{c}5 \text { Features: } \\
\text { Duration, protocol-type, } \\
\text { source bytes, and service } \\
\text { count }\end{array}$ & $\begin{array}{l}\text { Experimental } \\
\text { Trial-based. } \\
\text { (Manual) }\end{array}$ & $\begin{array}{c}\text { Both Binary } \\
\text { Classification and } \\
\text { Multiclass } \\
\text { Classification: } \\
\text { Normal, DoS, R2L, } \\
\text { Probe, U2R. }\end{array}$ & Flow-based & {$[252]$} \\
\hline $\begin{array}{c}\text { Anomaly detection } \\
\text { using payload analysis } \\
\text { in OpenFlow switches }\end{array}$ & $\mathrm{J} 48$ & KDD'99 & $\begin{array}{c}\text { Not } \\
\text { Mentioned }\end{array}$ & Not Mentioned & 41 Features & $\begin{array}{l}\text { Statistical } \\
\text { Analysis on } \\
\text { the features. }\end{array}$ & $\begin{array}{c}\text { Multiclass } \\
\text { Classification: } \\
\text { Normal, DoS, R2L, } \\
\text { Probe, U2R. } \\
\end{array}$ & $\begin{array}{c}\text { Packet- } \\
\text { based } \\
\text { Statistics- } \\
\text { based }\end{array}$ & {$[250]$} \\
\hline
\end{tabular}

Other than the reviewed ML-DL-based IDS solutions in SDN, researchers all over the world also adopted numerous other technologies to detect various attacks in the context of SDNs. For instance, statistics algorithms [37], [111], [253]-[255], [134], [144], [159], [167], [170]-[172], [226], similarity-based methods [256], graph model-based methods [257]-[259], third-party software like Snort [57], [94], [95], [260]-[262], threshold-based methods [51], [108], [254], [263], [264], fuzzy evaluations [265]-[267], inference-based methods [257], [268], blockchainbased methods [269]-[271], and honeynet-based methods [71], [272]-[274].

In the next section, we summarize the reviewed studies focusing on the learning categories of ML-DL algorithms, detection and mitigation approach, and hyperparameters of 
utilized ML-DL models as well as their performance evaluation metrics.

\section{TAXONOMY BASED SUMMARY OF EXISTING WORK}

Not all ML and DL algorithms are being used equally in developing IDS. By studying the literature, we have identified the commonly used ML and DL algorithms in designing IDS and thus we have developed a taxonomy of ML-DL algorithms utilized in IDS. The taxonomy is presented in Fig. 7. After reviewing all the existing works that use ML-DL algorithms, we have prepared Table XI that presents a comparative analysis of the existing works based on the ML-DL taxonomy presented in Fig. 7.

For this summarization, we have considered the learning techniques of the ML-DL algorithms, detection and mitigation approach, cross validation, train test split ratio, and various evaluation metrics such as accuracy, TPR, F1-score, recall, and FAR. Among different ML-DL algorithms, different supervised learning algorithms are commonly used with relatively low or medium complexity. On the other hand, different unsupervised algorithms, supervised and unsupervised algorithms together and reinforcement learning algorithms can achieve better accuracy with relatively higher complexities.

Based on different IDS techniques we have developed an IDS taxonomy that has been presented in Fig. 6. After studying the existing ML-DL based different IDSs, we can classify these IDSs using the IDS taxonomy presented in Fig. 6. In Table XII, we present the classification of the existing works based on the IDS taxonomy presented in Fig. 6 . In this table, all the existing works are categorized into five IDS types, such as flow-based, logbased, session-based, statistical analysis-based, and packetbased since all the existing works mainly focused on those types. Note that among different types of IDS flow-based IDSs are most common in SDN.

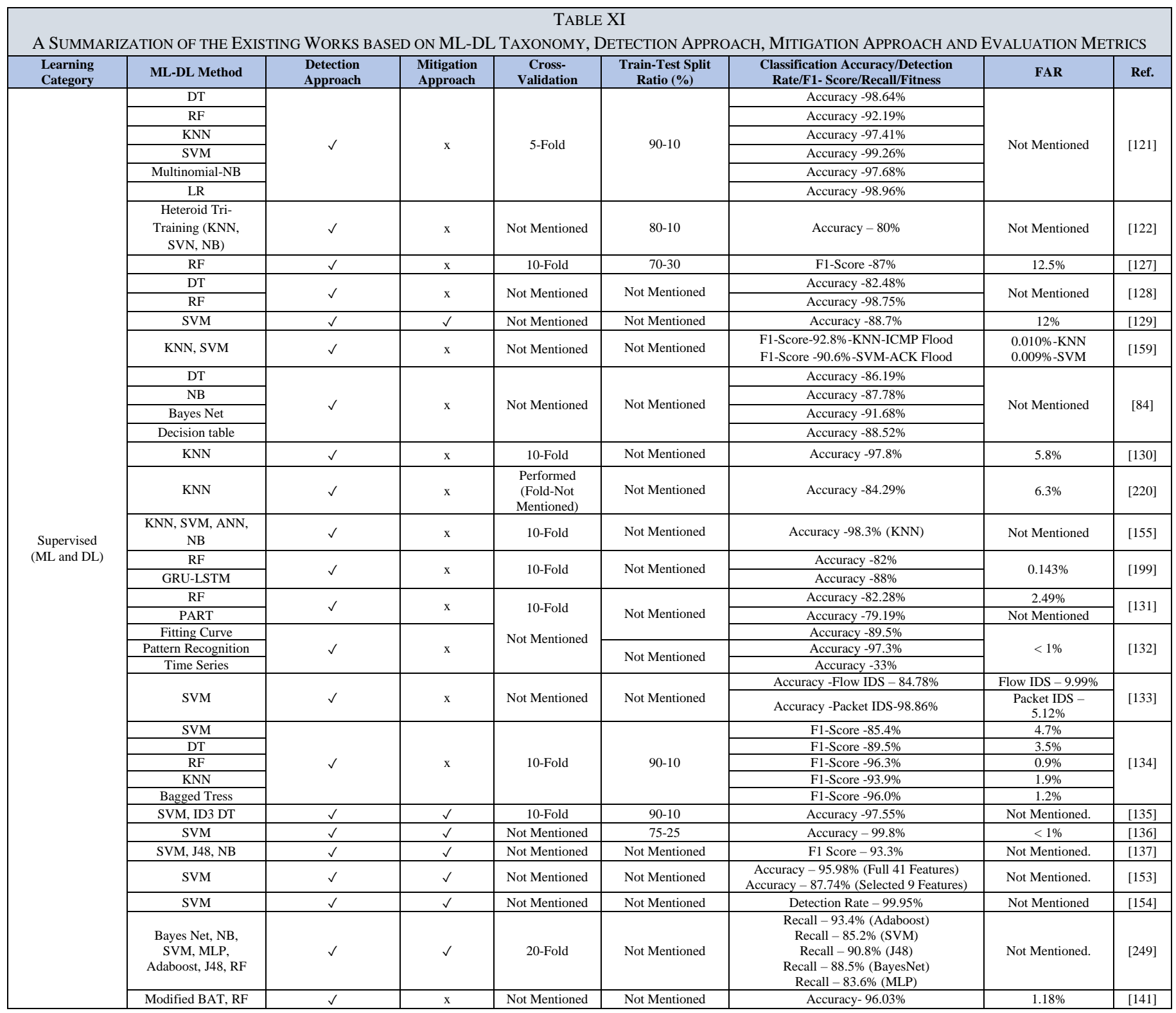




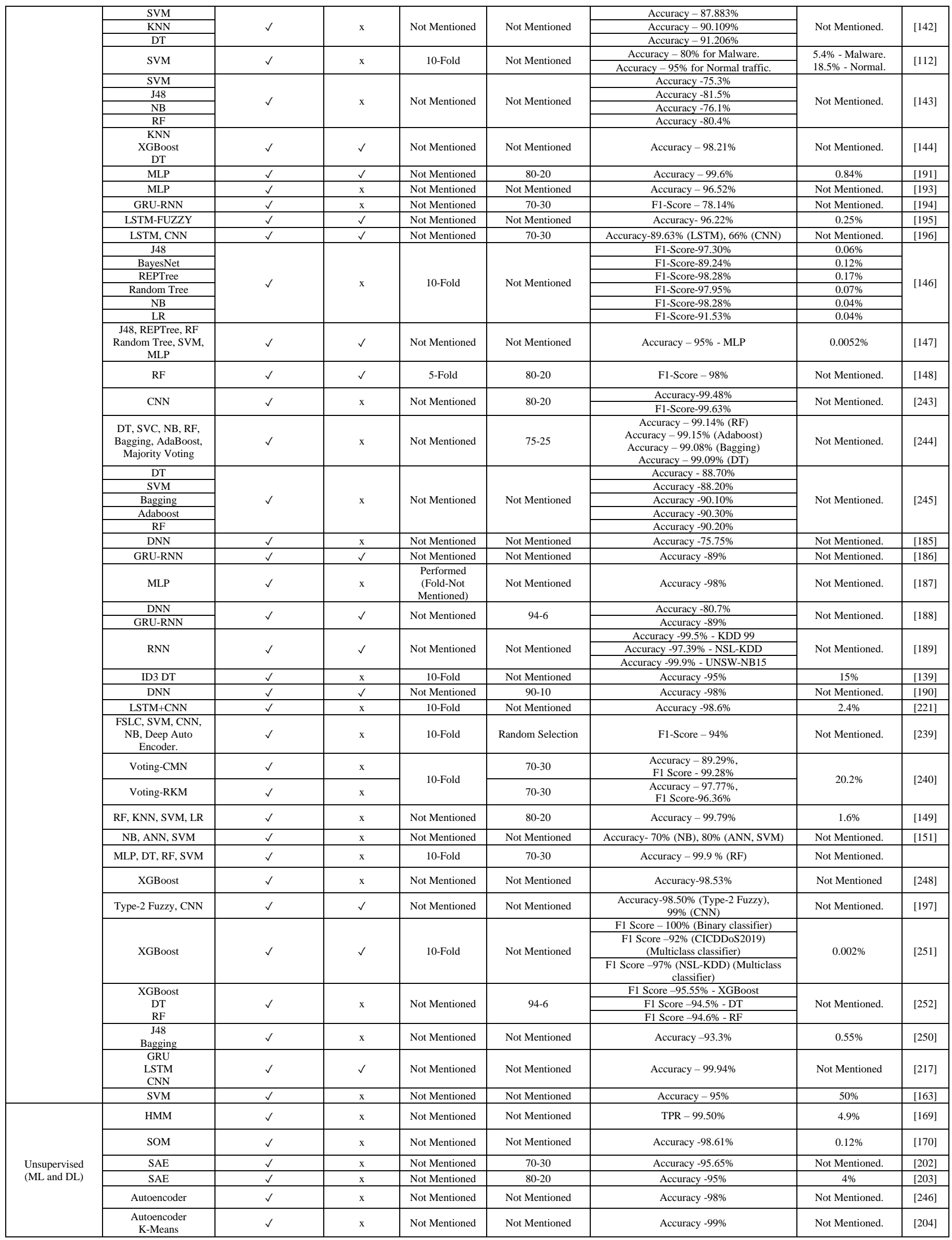




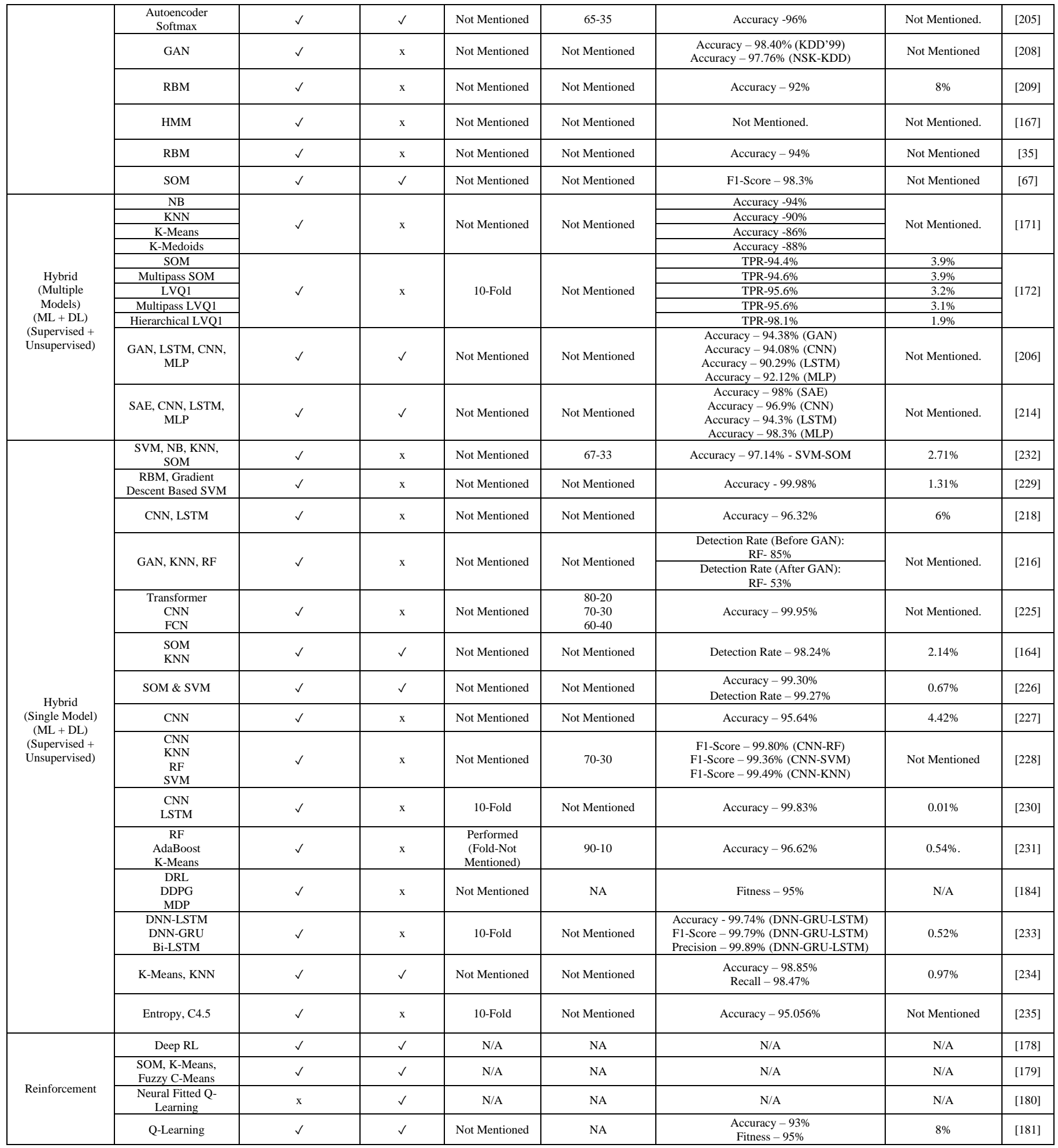

\section{BENCHMARK DATASETS IN IDS RESEARCH}

ML-DL method's role is to extract useful insight from data. Thus, based on the quality of input data the performance of MLDL method is enhanced. The foundation of the ML-DL approach depends on the in-depth understanding of data. The approved dataset for IDS must be simple to obtain and it must represent the host's activities or systems. However, creating a dataset is difficult and laborious too. Therefore, whenever a standard dataset is created, researchers from the entire world can reuse it in their work. A few most important of them are briefly described here.

DARPA1998: Developed by MIT's Lincoln laboratory, DARPA1998 dataset is a frequently used benchmark dataset in the IDS study. There are raw data packets along with labels in the dataset. Five classes of labels are available in this dataset, 


\begin{tabular}{|c|c|c|c|}
\hline \multicolumn{3}{|c|}{ TABLE XII } \\
\multicolumn{3}{|c|}{ CLASSIFICATION AND MAPPING OF THE REVIEWED WORKS BASED ON } \\
THE IDS AND ML-DL TAXONOMY
\end{tabular}

namely Normal, Probe, U2R, DoS, and R2L [275]. One of the limitations of this dataset is the existence of raw packets, as it cannot be applied explicitly to customary ML algorithms.

KDD'99: The most popular IDS evaluation dataset at present is the KDD99 dataset [277]. KDD99 was developed to solve the limitations of the DARPA1998 dataset. Its compilers derived forty-one (41) distinguishing features signifying the distinctiveness of network connections. The KDD99 labels are the same as the DARPA1998 labels. In KDD'99, there are four kinds of features: primary, content, host-based statistical aspect and statistical aspects dependent on time. However, The KDD'99 is an imbalanced dataset with duplicate data, which brings a bias factor in the result set. The significant amount of data replication prohibits detection methods from providing precise accuracy in attacks like R2L and U2R.

NSL-KDD: In the NSL-KDD dataset [278], data records of various groups are balanced, which eliminates the issue of bias problems in the classification task. Data records of NSL-KDD were taken from KDD99 without duplication. There are basically four types of features presented in NSL-KDD dataset, namely Basic, Content, Time-based, and Host-based features. Major drawback of this dataset is that it does not comprise any novel data items, and its data items are backdated to some extent and do not match the present network scenario. Thus, attacks like DoS can be detected smoothly, however, attacks like R2L and $\mathrm{U} 2 \mathrm{R}$ presents a considerable FAR.
Denial of Service (DoS): DoS is an attack intended to close down a device or network, thus making it unavailable to legitimate users. A basic illustration of a DoS attack is refusing rightful users access to a web service when the server is overwhelmed through multiple connection requests, e.g., SYN flood. A DoS attack's primary objective is not to obtain data but to delay or take down a website/service.

Remote to Local (R2L): With R2L attack, one tries to access a remote machine in an unauthorized manner, e.g., guessing a password using brute force technique by an automated script. R2L is usually initiated while legitimately accessing a local computer to acquire the root access unlawfully.

User to Root (U2R): The attacker begins with accessing the credentials of a standard user account on the device in the U2R attack and can leverage the weakness to obtain root privileges to the system. The attacker will access a regular user account using a social engineering method or by sniffing password and then exploiting flaws in the system to receive a super user's right, e.g., numerous buffer overflow attacks.

Probe: In probing, the attacker scans a network, a process called reconnaissance, to gain access to data or discover security flaws or identify the machines' deficiencies. These fraudulent activities conducted by Metasploit, a penetration testing software or the Nmap tools provide the intruders with knowledge about the possible targets of the attacks, e.g., port scanning, version scanning, vulnerability scanning etc.

The attack categories, along with their names, related services, mechanisms of the attacks, existing in the most commonly used DARPA1998, KDD99, and NSL-KDD dataset for IDS is presented in Table XIII.

\begin{tabular}{|c|c|c|c|}
\hline $\begin{array}{l}\text { LIST C } \\
\text { MEC }\end{array}$ & $\begin{array}{r}\text { TABI } \\
\text { ATTACK CATEGORIES WITH T } \\
\text { ANISM PRESENTED IN THE DAR } \\
\text { DATA }\end{array}$ & $\begin{array}{l}\text { XIII } \\
\text { EIR CORRESPOND } \\
\text { A1998, KDD'99 } \\
\text { STS. }\end{array}$ & $\begin{array}{l}\text { NG SERVICES AND } \\
\text { AND NSL-KDD }\end{array}$ \\
\hline Type & Name of the Attack & Services & Mechanism \\
\hline DoS & $\begin{array}{l}\text { Neptune, Pod, Teardrop, } \\
\text { Apache2, Back, Mailbomb, } \\
\text { UDP storm, Smurf, Land, } \\
\text { Process table, Arp poison, } \\
\text { Self ping, TCP reset, Ping of } \\
\text { death, Dos nuke, SSH } \\
\text { process table, SYN flood, } \\
\text { Crashiis, and Syslogd. }\end{array}$ & $\begin{array}{l}\text { HTTP, TCP, } \\
\text { ICMP, Syslog, } \\
\text { Echo. }\end{array}$ & $\begin{array}{l}\text { Bug, Misuse of } \\
\text { the feature. }\end{array}$ \\
\hline R2L & $\begin{array}{l}\text { Sendmail, Multihop, Guess } \\
\text { password, Spy, Xsnoop, Phf, } \\
\text { Imap, Snmpguess, Worm, } \\
\text { Warezmaster, Warezclient, } \\
\text { Xlock, Ftpwrite, ncftp, } \\
\text { netbus, netcat, ppmacro, } \\
\text { sshtrojan, and named. }\end{array}$ & $\begin{array}{l}\text { Telnet, Pop, } \\
\text { FTP, rlogin, } \\
\text { IMAP, HTTP, } \\
\text { SMTP, DNS. }\end{array}$ & $\begin{array}{l}\text { Misuse of the } \\
\text { feature, Bug, } \\
\text { Misconfiguration }\end{array}$ \\
\hline Probe & $\begin{array}{l}\text { Satan, Mscan, Saint, } \\
\text { Portsweep, resetscan, queso, } \\
\text { IPsweep, Insidesniffer, } \\
\text { Mscan, Ntinfoscan, and Is } \\
\text { domain. }\end{array}$ & $\begin{array}{l}\text { SNMP, ICMP, } \\
\text { TCP, HTTP. }\end{array}$ & $\begin{array}{l}\text { Misuse of the } \\
\text { feature. }\end{array}$ \\
\hline $\mathrm{U} 2 \mathrm{R}$ & $\begin{array}{l}\text { HTTP tunnel, Load module, } \\
\text { SQL attack, Rootkit, Perl, } \\
\text { Xterm, Buffer overflow, ps, } \\
\text { yaga, Eject, anypw, casesen, } \\
\text { sechole, ntfsdos, and Xterm. }\end{array}$ & User Session. & $\begin{array}{l}\text { Buffer overflow, } \\
\text { Bad management } \\
\text { of Temporary } \\
\text { file. }\end{array}$ \\
\hline
\end{tabular}


UNSW-NB15: This dataset includes nine diverse categories of attacks and a wide category of regular activities in practical life. Training set contains 175,341 records, and 82,332 records present in the test set collected from various forms, attacks, and regular records [279]. This dataset solves many of the problems faced by researchers using previous datasets resulting in a higher detection rate as well as faster training of the model.

ISCX 2012: To generate the dataset the authors used the concept of profiling [280]. The authors used two profiles: named Alpha and Beta. Alpha is used to generate traffic related to attack and Beta is used to generate regular traffic flow. DoS and Brute force attacks are two main attack types presented in this dataset. It has two formats: packet and flow. It contains data traffic containing an entire packet payload of the protocols: HTTP, SSH, IMAP, SMTP, FTP, and Post Office Protocol 3. Nevertheless, the features that were extracted from ISCX 2012 dataset are not quite enough for ML algorithm's proper evaluation. However, only HTTP traffic is included in the dataset, which does not represent modern traffic, in which most existing Internet traces are focused on HTTPS traffic [281].

CIC-IDS 2017: In [281], the authors proposed a new modernday network traffic-based dataset named CICIDS2017. It spans over 80 features of the network traffic. It contains two types of network traffic: normal and attack for five days in eight different files collecting attack types such as brute force, Botnet, PortScan, XSS, Infiltration, SQL injection, along with normal traffic. It contains whole packet payload with labeled flows in a PCAP format. One major drawback of CICIDS2017 dataset is that it has class imbalance issues [282]. The number of benign instances is way too much than other attack categories, which is almost $83.34 \%$. Thus, opens up the bias issue towards the benign traffic of the ML-DL-based IDS models in SDN.

CIDDS-001: CIDDS-001 [283], [284] is a flow-based labeled dataset. It comprises of three $\log$ files: attack logs, client configuration, client logs, also uses two servers, OpenStack server (e.g. mail, web, etc.) and External server (e.g. file synchronization, web server, etc.) [285] for data collection. DoS, PortScan, and Brute Force attacks are carried out on an emulated business network using the OpenStack environment to generate malicious traffic flow.

CSE-CIC-IDS2018: CSE-CIC-IDS2018 [286] is the newest dataset made accessible by the Canadian Institute for Cybersecurity in 2018/2019. The concept profiling was used to form this dataset [281]. This dataset takes into account profiles for human operators or agents responsible for creating network traffic events for numerous network protocols with varying topologies. In the dataset, two profiles were categorized, and five distinct attack mechanisms were applied. It employs two sorts of profiles: B-profiles and M-profiles. The B-profiles include user behavior such as distribution of packet sizes, number of packets in each flow, patterns and size of the payload, and request time distribution of protocols including FTP, HTTP, HTTPS, IMAP, POP3, SMTP, and SSH. An M-Profile, on the other hand, unambiguously depicts a security attack scenario. The dataset includes attack scenarios for security threats such bruteforce, web attacks, botnets, and DDoS. With 80 features, it also contains each machine's log and collected network traffic [5].

CTU-13: This dataset was generated in CTU University, Czech Re-public [287]. It is one of the most comprehensive labeled datasets accessible, containing botnet traffic as well as regular and background labeled data [259]. The dataset includes botnet traffic as well as regular and background communication traffic. The traffic capture procedure comprises 13 separate scenarios of different botnet samples.

InSDN: The InSDN dataset [228] is regarded as one of the first to build a comprehensive dataset for evaluating IDSs specific to SDN paradigm. The InSDN dataset's regular traffic includes standard application services, including HTTP, HTTPS, FTP, DNS, Email, and SSH. DoS, DDoS, Probe, Botnet, Exploitation, Password-Guessing, and Web attacks are among the attack types this dataset addresses. Furthermore, these attacks come from a variety of internal and external sources to mimic real-world attack scenarios. The dataset contains a total of 361317 occurrences of normal and attack traffic, where 68424 occurrences are normal traffic and 292893 occurrences are attack-oriented traffic [228].

CIC-DDoS-2019: CIC-DoS-2019 is a novel dataset that includes a wide range of DDoS attacks. This dataset includes 28 typical network behaviors as well as the most recent prevalent forms of DDoS attacks [206]. The data has been divided into two sections, one for training and the other for testing. For Training, WebDDoS, NetBIOS, LDAP, MSSQL, SNMP, UDP-Lag, DNS, SYN, SSDP, NTP, UDP, and TFTP DDoS attacks were executed and for testing they have executed the SYN, NetBIOS, MSSQL, LDAP, UDP, UDP-Lag and PortScan attacks. Some other notable datasets that are used in SDN-IDS research are CAIDA, Hogzilla, and ISOT.

A summary of the aforementioned public benchmark datasets is presented in Table XIV. The comparison was done based on the data collection process, labeling, formatting, number of features, attack variant and their class balance issues of the collected data

Table XV summarizes the reviewed studies that used the benchmark public datasets discussed in section $\mathrm{X}$ and the assessment criteria/metrics listed in Table $\mathrm{X}$ to evaluate the efficacy of their proposed solutions. 


\begin{tabular}{|c|c|c|c|c|c|c|c|c|c|c|c|c|}
\hline \multicolumn{13}{|c|}{$\begin{array}{c}\text { TABLE XIV } \\
\text { IC BENCHMARK DATASETS UTILIZED IN IDS: }(\sqrt{ }: \text { YES, } x: \text { NO) }\end{array}$} \\
\hline Dataset & Number of & Feature & Label & Metadata & Format & Attack & Number and Name of & \multirow{2}{*}{$\begin{array}{l}\text { Traffic } \quad+ \\
\text { Network Type }\end{array}$} & \multirow[t]{2}{*}{ Balanced } & \multicolumn{2}{|c|}{ Instances } & \multirow[t]{2}{*}{ 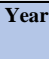 } \\
\hline & Features & Extraction Tool & & & & Diversity & Available Attack Types & & & Training & Testing & \\
\hline DARPA & $\begin{array}{l}\text { TCP } \\
\text { Connections } \\
\& \quad \text { High- } \\
\text { level } \\
\text { Features. }\end{array}$ & $\begin{array}{l}\text { Raw files } \\
\text { Features need to } \\
\text { be extracted by } \\
\text { the researchers. }\end{array}$ & $\checkmark$ & $\mathrm{x}$ & $\begin{array}{l}\text { Data, } \\
\text { Packet, } \\
\text { Logs }\end{array}$ & $\checkmark$ & $\begin{array}{l}4 \\
\text { DoS, Probe, U2R, and } \\
\text { R2L. }\end{array}$ & \multirow{4}{*}{$\begin{array}{l}\text { Emulated } \\
\text { Traffic } \\
+ \\
\text { Small-Scale } \\
\text { Network }\end{array}$} & $\mathrm{x}$ & $\begin{array}{l}\text { Not } \\
\text { Mentioned }\end{array}$ & 201 & 1998 \\
\hline KDD99 & 41 & Pre-processed & $\checkmark$ & $\mathbf{x}$ & Other & $\checkmark$ & $\begin{array}{l}4 \\
\text { DoS, Probe, U2R, and } \\
\text { R2L. }\end{array}$ & & $\mathrm{x}$ & 4898431 & 311029 & 1998 \\
\hline NSL-KDD & 41 & Pre-processed & $\checkmark$ & $\mathbf{x}$ & Other & $\checkmark$ & $\begin{array}{l}4 \\
\text { DoS, Probe, U2R, and } \\
\text { R2L. }\end{array}$ & & $\mathrm{x}$ & 125973 & 22544 & 2009 \\
\hline $\begin{array}{l}\text { UNSW- } \\
\text { NB15 }\end{array}$ & 49 & $\begin{array}{l}\text { Argus and Bro- } \\
\text { IDS Tools [288] }\end{array}$ & $\checkmark$ & $\mathbf{x}$ & $\begin{array}{l}\text { Packet } \\
+ \text { Other }\end{array}$ & $\checkmark$ & 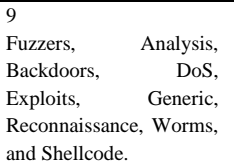 & & $\mathrm{x}$ & 175341 & 82332 & 2015 \\
\hline ISCX 2012 & 20 & Not Mentioned & $\checkmark$ & $\checkmark$ & $\begin{array}{l}\text { Packet } \\
\text { + Flow }\end{array}$ & $\mathrm{x}$ & $\begin{array}{l}4 \\
\text { Brute Force, SSH, } \\
\text { Infiltrating, HTTP DoS, } \\
\text { and DDoS. }\end{array}$ & $\begin{array}{l}\text { Realistic } \\
\text { network traffic } \\
+\quad \text { Small-Scale } \\
\text { Network }\end{array}$ & $\mathrm{x}$ & 1167479 & 629274 & 2012 \\
\hline $\begin{array}{l}\text { CIC- } \\
\text { IDS2017 }\end{array}$ & 83 & $\begin{array}{l}\text { CICFlow-Meter } \\
\text { [289] }\end{array}$ & $\bar{v}$ & $\bar{v}$ & $\begin{array}{l}\text { Packet } \\
+ \text { Flow }\end{array}$ & $\checkmark$ & $\begin{array}{l}7 \\
\text { Web based, Brute force, } \\
\text { DoS, DDoS, Infiltration, } \\
\text { Heart-bleed, Bot, and } \\
\text { PortScan. }\end{array}$ & $\begin{array}{l}\text { Realistic } \\
\text { network traffic } \\
+\quad \text { Small-Scale } \\
\text { Network }\end{array}$ & $\mathbf{x}$ & & & 2017 \\
\hline CIDDS-001 & 14 & Not Mentioned & $\checkmark$ & $\checkmark$ & Flow & $\checkmark$ & $\begin{array}{l}4 \\
\text { PortScan, } \quad \text { PingScan, } \\
\text { Brute-Force, and DoS. }\end{array}$ & $\begin{array}{l}\text { Both } \text { Emulated } \\
\text { and Realistic } \\
\text { network Traffic } \\
+ \\
\text { Small-Scale } \\
\text { Network }\end{array}$ & $\mathrm{x}$ & & & 2017 \\
\hline $\begin{array}{l}\text { CSE-CIC- } \\
\text { IDS2018 }\end{array}$ & 83 & CICFlow-Meter & $\checkmark$ & $\mathrm{x}$ & $\begin{array}{l}\text { Packet } \\
\text { +Flow }\end{array}$ & $\checkmark$ & $\begin{array}{l}7 \\
\text { Web based, Brute force, } \\
\text { DoS, DDoS, Infiltration, } \\
\text { Heart-bleed, Bot, and } \\
\text { PortScan. }\end{array}$ & 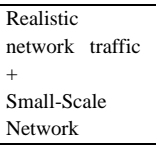 & $\mathbf{x}$ & & & 2018 \\
\hline CTU-13 & $\begin{array}{l}\text { Flow-based } \\
\text { Features }\end{array}$ & Argus [290] & $\checkmark$ & $\checkmark$ & Packets & $\checkmark$ & $\begin{array}{l}8 \\
\text { IRC, SPAM, CF, PS, } \\
\text { HTTP, DDoS, P2P } \\
\text { Botnet, and US. [291] }\end{array}$ & $\begin{array}{l}\text { Realistic } \\
\text { network traffic } \\
+ \\
\text { Small-Scale } \\
\text { Network }\end{array}$ & $\mathrm{x}$ & & & 2014 \\
\hline InSDN & 83 & CICFlow-Meter & $\checkmark$ & $\checkmark$ & $\begin{array}{l}\text { Packet } \\
+ \text { Flow }\end{array}$ & $\checkmark$ & $\begin{array}{l}7 \\
\text { U2R, Web attack, Probe, } \\
\text { Password guessing, DoS } \\
\text { (HULK, TorshHammer, } \\
\text { HTTP-Flood), DDoS, } \\
\text { and Botnet. }\end{array}$ & $\begin{array}{l}\text { Realistic } \\
\text { network traffic } \\
+ \\
\text { Small-Scale } \\
\text { Network }\end{array}$ & $\mathrm{x}$ & & & 2020 \\
\hline $\begin{array}{l}\text { CIC- } \\
\text { DDoS2019 }\end{array}$ & 87 & CICFlow-Meter & $\checkmark$ & $\checkmark$ & $\begin{array}{l}\text { Packets } \\
\text { +Flow }\end{array}$ & $\checkmark$ & $\begin{array}{l}12 \\
\text { WebDDoS, NetBIOS, } \\
\text { LDAP, MSSQL, SNMP, } \\
\text { UDP-Lag, DNS, SYN, } \\
\text { SSDP, NTP, UDP, and } \\
\text { TFTP. }\end{array}$ & $\begin{array}{l}\text { Realistic } \\
\text { network traffic } \\
+ \\
\text { Small-Scale } \\
\text { Network. }\end{array}$ & $\mathrm{x}$ & & & 2019 \\
\hline
\end{tabular}

TABLE XV

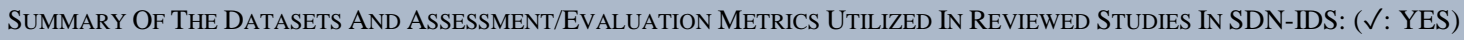

\begin{tabular}{|c|c|c|c|c|c|c|c|c|c|c|c|c|c|c|c|c|c|c|c|c|c|c|c|c|}
\hline \multirow{3}{*}{ Ref. } & \multicolumn{13}{|c|}{ Datasets } & \multicolumn{11}{|c|}{ Assessment/Evaluation Metrics } \\
\hline & \multicolumn{13}{|c|}{$\begin{array}{l}\text { NSLKDD - NK, KDD CUP' } 99 \text { - KD, UNSWNB15 - UN, InSDN - IN, CICIDS2017 - C7, } \\
\text { CSECICIDS2018 - C } 8 \text {, ISCX } 2012 \text { - IS, DARPA1998 - DA, CIDDS001 - C1, CTU13 - } \\
\text { C9, CICDDoS - C10, Self-Collected/Simulated - SC, Others - OT (HogZilla, ISOT, } \\
\text { CAIDA etc.) }\end{array}$} & \multicolumn{11}{|c|}{$\begin{array}{l}\text { Accuracy - ACC, Precision - PR, F-Measure - F1, Recall - RC, Receiver Operating } \\
\text { Characteristic - ROC, Precision Recall Curve - PRC, Area Under the Curve - AUC, } \\
\text { Fitness - FT, True Positive Rate - TPR, False Alarm Rate - FAR, Others - OT (Mean } \\
\text { Absolute Error, Reward, Root Mean Squared Error, CPU Utilization, Bandwidth } \\
\text { Involvement etc.) }\end{array}$} \\
\hline & NK & KD & UN & IN & $\overline{\mathrm{DA}}$ & IS & $\begin{array}{l}\mathrm{C} \\
1\end{array}$ & & & $\begin{array}{l}\mathrm{C} \\
9 \\
\end{array}$ & $\begin{array}{l}\mathrm{C} \\
10 \\
\end{array}$ & OT & SC & $\mathrm{ACC}$ & PR & F1 & $\mathrm{RC}$ & ROC & PRC & AUC & TPR & FAR & FT & OT \\
\hline [67] & & & & & & & & & & & & & $\checkmark$ & & & $\checkmark$ & & & & & & & & $\checkmark$ \\
\hline$[121]$ & & & & & & & & & & $\checkmark$ & & $\checkmark$ & & $\checkmark$ & $\checkmark$ & $\checkmark$ & $\checkmark$ & $\checkmark$ & $\checkmark$ & & $\checkmark$ & & & \\
\hline$[122]$ & & & & & & & & & & & & $\checkmark$ & & & $\checkmark$ & $\checkmark$ & & & & $\checkmark$ & & & & \\
\hline [127] & & & & & & & & & & & & $\checkmark$ & & & & $\checkmark$ & & $\checkmark$ & & $\checkmark$ & & $\checkmark$ & & \\
\hline$[128]$ & & $\checkmark$ & & & & & & & & & & & & $\checkmark$ & $\checkmark$ & $\checkmark$ & $\checkmark$ & & & & & & & \\
\hline [129] & & & & & & & & & & & & $\checkmark$ & & $\checkmark$ & $\checkmark$ & $\checkmark$ & $\checkmark$ & & & & $\checkmark$ & $\checkmark$ & & \\
\hline
\end{tabular}




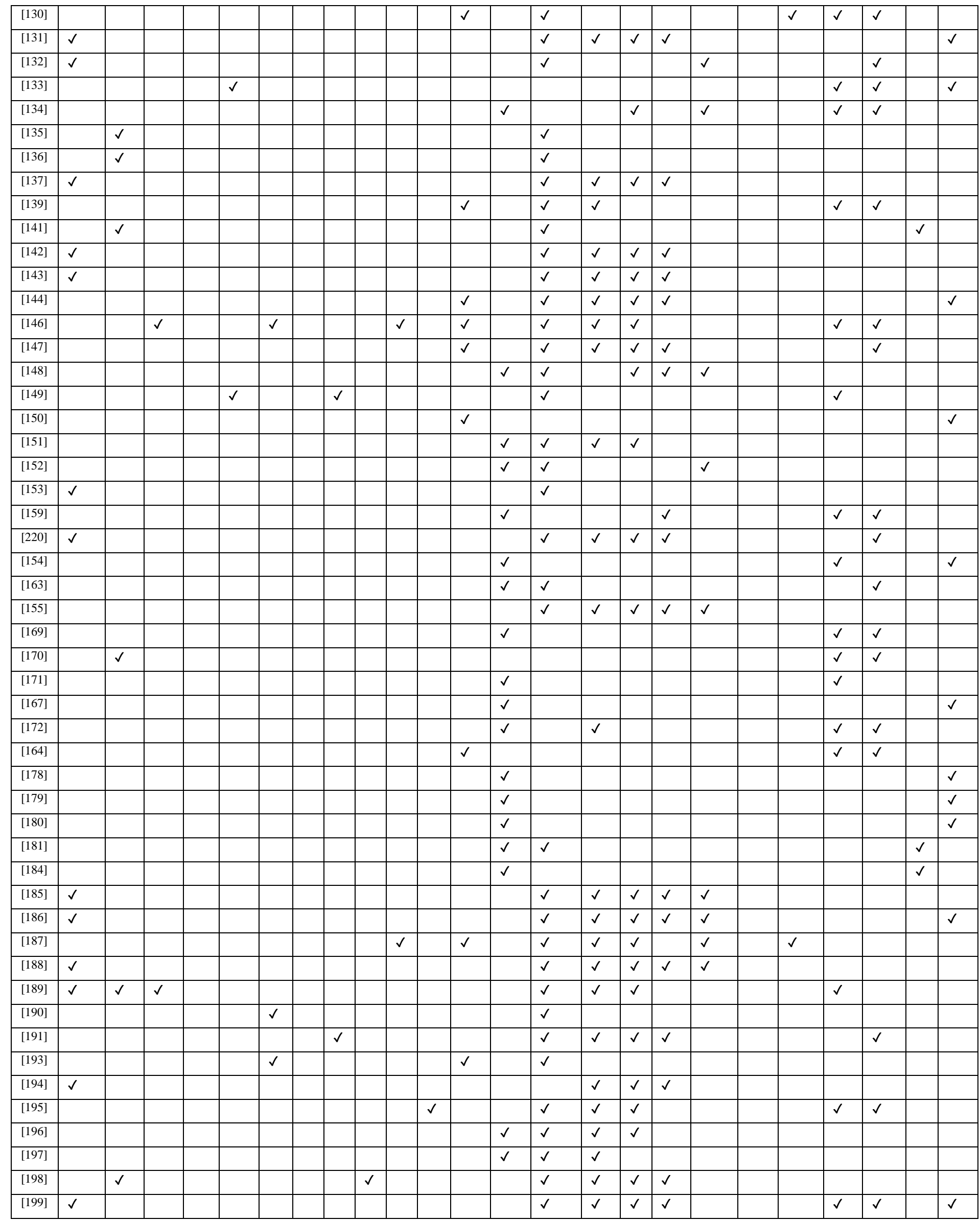




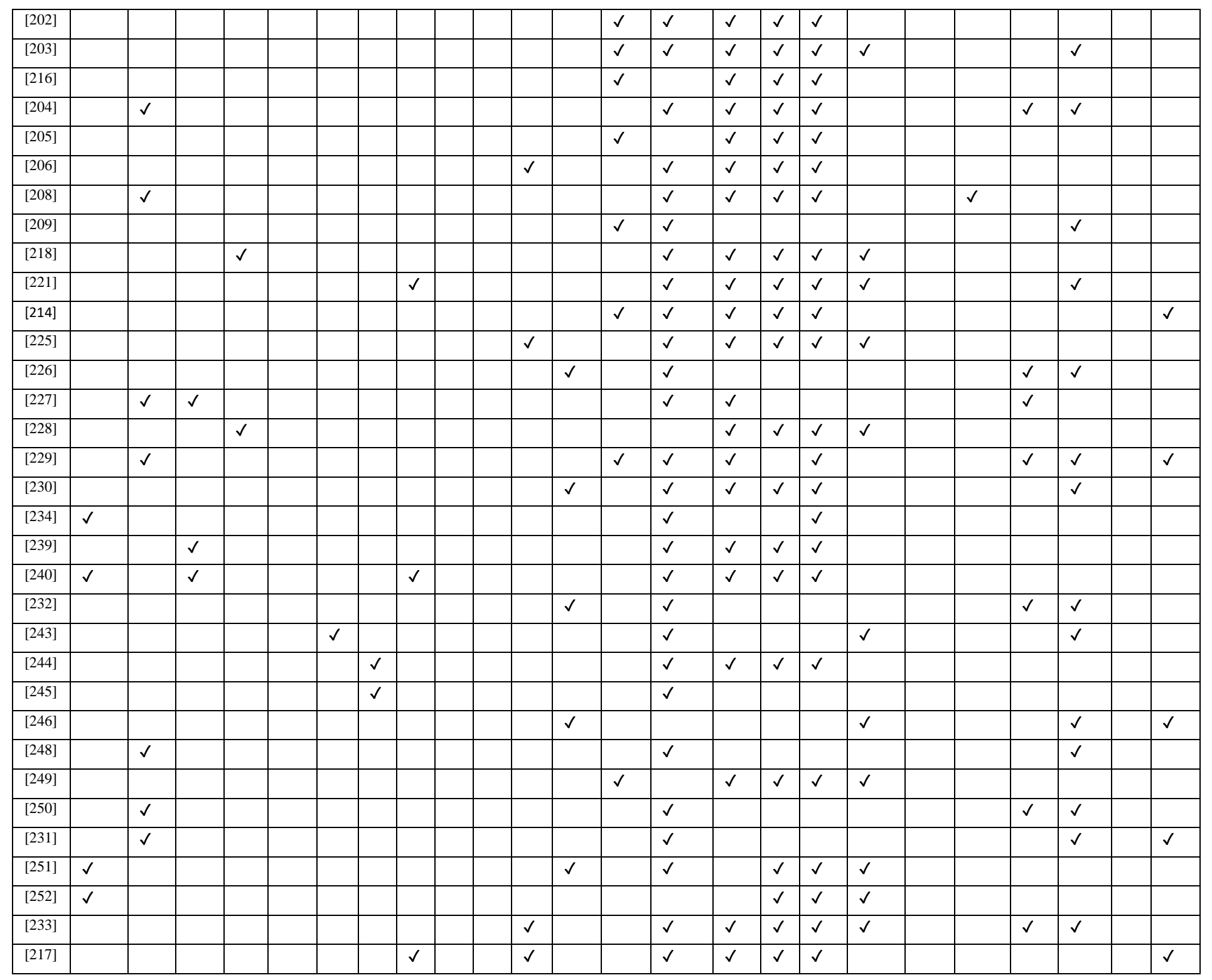

\section{Challenges, IsSues, InSightS AND Future RESEARCH DIRECTIONS}

Many researchers in SDN paradigm have explored Machine Learning and Deep Learning-based IDS solution in the past few years. However, many important issues remain unexplored. In this section, we address some of the challenges that are faced while developing an efficient ML-DL-based IDS in SDN.

\section{Single Controller Issue}

A centralized control layer is included in the SDN design for increased network control, programmability, and management. However, the lack of a robust and secure controller platform makes this centralization vulnerable to various attacks [68]. The controller is the principal target of the attackers. Since network congestion might cause buffer overflows in OF switches, SDN may encounter control packet loss. Control packet loss impairs the network policies' reliability. The solution of how a single controller can maintain network information effectively and consistently in the event of control packet loss remains an open issue [292]. By delivering unknown packets to OF-based switches, SDN controllers might be overloaded. When a packet reaches an OF-based switch, it is forwarded according to the rule defined in the OpenFlow switches' flow table. The packet is passed to the controller for further action if no matching flow entry is detected. The controller then selects how to deal with the packet and, if necessary, adds a new rule to the OF-based switch. Vast volumes of such traffic directed at SDN controllers might take the controller down and consume a large amount of bandwidth on the communication link between switches and controllers in the SDN paradigm [68]. The majority of the studies have deployed only a single controller in the SDN topology, such as NOX, POX, ONOS, RYU, OpenDayLight, [128], [132], [181], [220], [248], [251]. Plus, managing the network through 
this centralized controller creates a considerable overhead for the control plane in SDN. Thus, to reduce this overhead, multiple controllers in the control plane can be an option for future IDS solutions in SDN to distribute the overhead among controllers, thus minimizing the overhead. Some studies have adopted this multi-controller technique in the control plane of SDN architecture [293]-[296], nevertheless, maintaining a wellorganized synchronization among the controllers is also a research gap that needs addressing. However, a pool of controllers may be accessed by applications from multiple networks. If an adversary impersonates the controllers or applications associated with it, it can easily access network resources and eventually disrupt network operations. The adversary may misconfigure the controllers, causing network performance to suffer, reducing the degree of reliability, that is another open research area [297]-[299]. Communication links between switches and controllers must be protected in the same way. Any attack on the controller-to-switch communication link has the potential to inflict significant network damage [300].

\section{Bottleneck Creation Due to Lack of Scalability, Evaluation and Testing}

SDN was initially designed for a low-scaled network, but with increasing network architecture size over time, the controller could face significant problems in a large number of incoming and outgoing flow statistics, thus creating a bottleneck. With the increased network size, there is always a chance for congestion and attacks between the controllers and switches. This lack of SDN scalability might prove costly for an automated ML-DLbased IDSs operation. Hence, it is important to associate security and scalability in SDN to create secure SDN-based architectures. Furthermore, fellow researchers set up their suggested experimental settings on a single machine, which is an obstacle in evaluating and verifying security measures. As a result, another open issue is using multiple physical computers to evaluate suggested security measures in the SDN paradigm. The Mininet emulator was used to replicate and test the majority of the reviewed ML-DL-based IDS solutions, such as [167], [171], [189], [191], [234], [246]. On the one hand, a Mininet simulator makes prototyping an SDN easier and may be used to illustrate the concept of the proposed system. However, it does place restrictions on network designs, sizes, and functionalities, which brings the discussion about the scalability issue. Compared to the Mininet environment, deploying such a solution in real-life network infrastructure is costly, time-consuming, and challenging. A hardware-based IDS implementation, on the other hand, is another method of analyzing and verifying the proposed IDS mechanism.

\section{Scarcity of Proper Model Selection Study}

To create any ML-DL-based model, it is necessary to hypertune the model to find the best parameters for effective training.
However, we have found an apparent lack of study in all the research papers, especially IDSs that are DL based and did not mention much about the hyper-tuning parameters to train the model. Some papers [147], [152], [186], [189], [203], [221] mentioned finding the useful hyperparameters using the grid search technique. Though feature selection significantly influences IDS' performance, only a few researchers use it as the primary component of their studies. When constructing an IDS for SDN, most researchers overlook the feature selection process. As per our study, although SDN presents the opportunity for many more features to be extracted from the network traffic or the applied dataset, the most notable features used in various attack detection systems in SDN are the number of packets per flow, number of hosts, protocol, source, and destination port number, number of bytes per flow, flow duration, server count, entropy of the source, and destination IP addresses, and entropy of source, and destination ports, and flags, which are selected based on the researchers' individual choice, experience or simply being encouraged from previous studies. Most of the datasets used by the researchers do not adequately represent the actual flow-based architecture of SDN. Although some studies performed feature selection and discussed about its significance in the development of ML-DL-based IDS [181], [234], [281], to enhance the effectiveness and scalability of IDS, dynamic selection and continuous updating of SDN-based features are still a necessity.

\section{Lack of SDN Specific Dataset for Model Training}

Utilizing non-specific SDN datasets may result in a compatibility issue because the deployment of attack vectors must take into account the network's new architecture [301]. Most of the researchers have used publicly available datasets like KDD99, NSL-KDD, UNSW-NB15, etc. The attacks presented in those datasets are not adequately suited for SDN architecture. Most of the attacks are outdated, and attack types suffers imbalance issue, majority of the traffic is labeled as normal, as shown in Table XIII. Thus, ML-DL-based IDS trained on those datasets shows a high FAR due to its inefficiency in detecting novel attack patterns. Cyber hackers are continually attempting to infiltrate in a new and more effective way, which may not prove fruitful with ML- DL-based IDS models trained on historical datasets. A potential strategy to fix the problem by creating new attacks is the GAN. Self-collected network traffic data used by researchers are generated using a synthetic network paradigm, thus lacking real-time efficacy, and brings the discussion about biases in the datasets as the tools used to create those synthetic datasets cannot replicate real network traffic. A research gap in the present research work is the training of machines with accurate normal behavior-based traffic data. Data collection and processing feature selection plays an essential role in any ML-DL-based IDS model to train better and reduce the overall complexity. Features collected from the publicly 
available datasets are not SDN specific. Feature extraction and selection is one of the most important part of any ML-based solutions, however, this core part of ML was not mentioned by many reviewed papers in this survey, such as [133], [144], [171], [178], [180]. One noticeable point of the review is the lack of established reasoning regarding data collection, feature extraction, and feature selection and their suitability with SDN in papers where synthetic network data was used. As a result, more study is needed in generating public datasets specific to SDN regarding consistent and precise IDS solution.

Lack of Diverse Attack Detection and Additional Focus Towards Detection Rather Than Mitigation and Prevention Approach

With the advancement of technology along with the increasing number of malicious hackers, more and more different types of attacks are being generated. In SDN, whenever a switch cannot find a flow that matches a specific packet, it sends a "packet_in" instruction for the packet to the SDN controller. Attackers can create loads of packets with new flows and attack the network by flooding it with these packets, creating a DoS attack. Current SDN specific IDSs inspect the first packet of every flow and proceeds with the same decision for the next packets in the same flow. An attacker could exploit this characteristic by sending malicious packets after the first one. If any attacker attacks by combining two different types of attack in a single packet, the controller might match only with the known attack types, the unknown one might be undetected. One evident shortcoming of some of the reviewed papers [84], [128]-[130], [136], [152], [190], [240], [243] is their binary attack detection mode. Most of them can only identify between normal flow and DDoS attack like TCP SYN Flood, ICMP Flood, etc. However, new attack categories are affecting SDN architecture. Aside from typical DDoS attacks, new forms of DDoS attacks are emerging all the time. These new kinds of DDoS attacks utilize a different approach than regular DDoS attacks. For example, the link flooding attack, which seeks to flood network links rather than servers, and the Crossfire attack targets the servers around the target server rather than the target server itself. Many forms are taken by these DDoS attacks in the network, which implies that typical DDoS detection techniques may be unable to identify them [16]. Even though some researchers have made efforts to identify DDoS and MITM attacks quickly and early in the process, such as [65], [148], additional study on developing DDoS and MITM detection mechanisms to detect attacks early is needed. GAN and Variational Auto Encoders (VAE) can be implemented to augment the dataset to increase the diversity of the intrusion in DL based IDS. Hence, more research and indepth study are needed to detect novel attack types in SDN using ML-DL-based IDS. Another point is that, most of the ML-DLbased solutions focused on the intrusion detection approach only, few have discussed about the mitigation approach along with the detection approach, such as [129], [144], [147], [188], [205], [249], but there is a clear lack of studies which discussed about the prevention mechanism along with the detection and mitigation. We think that preventing the attack and keeping the SDN-based system functional while under attack is more critical than detecting or mitigating the attack. In the prevention phase, the attack is prevented from expanding into the network before it consumes network resources and necessitates detection and mitigation procedures. The prevention mechanism provides fairness among the connected hosts regarding proper bandwidth allocation, energy consumption, and request handling. Thus, more studies and required to prevent the attack in SDN-enabled infrastructure rather than detection and mitigation only [15].

\section{Lack of Low-Rate DDoS Attack Detection}

One noticeable point from this survey is that most current research aims to detect high-rate DDoS attacks. A minor amount of study has been done on detecting low-rate DDoS attacks. A low-rate DDoS attacker makes use of TCP's congestion-control vulnerability by frequently sending burst attack packets over short periods or continually sending attack packets at a low rate [302]. A low-rate DDoS attack would deliver a smaller number of packets than a high-rate DDoS attack; as a result, features that may represent a high-rate DDoS attack will be inappropriate for identifying a low-rate DDoS attack [16]. As a result, detecting a low-rate DDoS attack is still challenging and needs more attention. Though some studies [147], [169], [226], [303] have tried to detect low-rate DDoS in SDN using ML-based approaches, more research is needed in this area.

\section{Lesser Exploration of Unsupervised DL approaches}

Most of the fellow researchers focused on supervised MLDL algorithms to build an IDS solution for SDN environment, such as [121], [130], [148], [189], [221]. Unsupervised DL algorithms such as GAN, VAE, SAE, and Sparse AutoEncoder have not been extensively explored in the IDS solution in the SDN paradigm. It is confounding to generate attack signatures, definitions, states, or even IDS policies relevant to the dynamic nature of SDN. GAN networks are particularly good at forming adversarial examples that can trick IDS models in SDN. Hence, investigation regarding unsupervised DL method GAN, SAE, VAE, Sparse, and Denoising AutoEncoder should be explored more to generate synthetic attack signatures and train the SDN to distinguish diverse attack categories of different kinds. There is a shortage of SDN-specific datasets in the literature, and the procedure of obtaining SDN-specific labeled data is costly and requires manual labeling by network professionals. As a result, research into the development of unsupervised learning algorithms for intrusion detection systems is worthwhile. Another noticeable point is that, most of the ML-DL-based IDS solution are evaluated using the conventional accuracy, precision, and F-measure metrics. However, only a few studies 
[131], [226], [229], [304] have highlighted the throughput, bandwidth involvement, energy consumption, and CPU utilization of the SDN controller.

\section{CONCLUSIONS}

Traditional networking control is shifted from hardware to software with the utilization of SDN-enabled infrastructure. The network operations and management are simplified as a result of this adjustment. Network designers' focus turns away from coding related to the low-level device configurations and toward developing a software-based solution that allows for network administration and troubleshooting. On the negative side, SDN's decoupled design through the separation of data plane and control plane has added to the network's security issues. SDN introduces new areas of threats and attacks due to its programming capabilities and centralization of the controller.

This survey provides a comprehensive overview of the studies conducted on developing automated IDS utilizing various MLDL algorithms in the SDN paradigm. Due to the centralized control of the entire SDN, security carries a greater significance. Integration of a large number of novel attacks makes this centralized control a point of significant vulnerability too. Hence, necessary security measures are needed to identify attacks in the SDN. From our review, it is clear that the DoS attacks are considered the most noteworthy extrinsic threats in SDN. Since the attacks essence is dynamic, numerous aspects should be considered when implementing IDS in SDN. As a result, the detection process must be adaptable to the varying attack types. Numerous ML-DL-based methods are extensively used in developing IDS by training a model to distinguish normal activities and intrusions.

This survey evaluates the SDN paradigm's challenges, issues, and future research prospects by reviewing current ML-DLbased IDS studies and provides a direction of references to other researchers performing meticulous investigations. In this survey, we have provided an overview of these methods and provided a comparative analysis of the studies conducted based on their learning category, datasets used in the study, feature selection process, utilized controller, attack classification types, and achieved performance in detecting attacks in SDN. SDN related research challenges, issues, and future research directions were also briefly analyzed and examined. Most of the researches are based on utilizing SL-based ML-DL algorithms for developing IDS solution in SDN. The attacks are evolving day by day. With the lack of SDN specific datasets, the field of UL-based methods is a clear scope of further research to deal with unknown attack types. Finally, we discussed some research problems and obstacles for prospective researchers working on a new SDNbased IDS solution. The field of applying ML-DL algorithms to SDN is vast, and there are numerous challenges ahead. Nonetheless, it is in the network community's best interests to resolve the problems and move forward. We strongly believe that this survey will help future researchers using ML-DL methods to build IDS solutions as a guideline to secure the SDN paradigm.

\section{REFERENCES}

[1] H. J. Liao, C. H. Richard Lin, Y. C. Lin, and K. Y. Tung, "Intrusion detection system: A comprehensive review," J. Netw. Comput. Appl., vol. 36, no. 1, pp. 16-24, 2013.

[2] "Software Defined Networking Market Size, Share and Global Market Forecast to 2025 | COVID-19 Impact Analysis," Marketsandmarkets.com, 2020. [Online]. Available: 14 Feb 2021.

[3] D. Kreutz, F. M. V. Ramos, P. E. Verissimo, C. E. Rothenberg, S. Azodolmolky, and S. Uhlig, "Software-defined networking: A comprehensive survey," Proc. IEEE, vol. 103, no. 1, pp. 14-76, 2015. W. Xia, Y. Wen, C. H. Foh, D. Niyato, and H. Xie, "A Survey on Software-Defined Networking," IEEE Commun. Surv. Tutorials, vol. 17, no. 1, pp. 27-51, 2015.

[5] S. W. Lee et al., "Towards secure intrusion detection systems using deep learning techniques: Comprehensive analysis and review," J. Netw. Comput. Appl., vol. 187, no. December 2020, p. 103111, 2021. H. Liu and B. Lang, "Machine learning and deep learning methods for intrusion detection systems: A survey," Appl. Sci., vol. 9, no. 20, 2019. P. Mishra, V. Varadharajan, S. Member, and U. Tupakula, "A Detailed Investigation and Analysis of using Machine Learning Techniques for Intrusion Detection," IEEE Commun. Surv. Tutorials, vol. 21, no. 1, pp. 686-728., 2018.

[8] A. Khraisat, I. Gondal, P. Vamplew, and J. Kamruzzaman, "Survey of intrusion detection systems: techniques, datasets and challenges," Cybersecurity, vol. 2, no. 1, 2019.

[9] Z. Ahmad, A. Shahid Khan, C. Wai Shiang, J. Abdullah, and F. Ahmad, "Network intrusion detection system: A systematic study of machine learning and deep learning approaches," Trans. Emerg. Telecommun. Technol., vol. 32, no. 1, pp. 1-29, 2021.

[10] N. Sultana, N. Chilamkurti, W. Peng, and R. Alhadad, "Survey on SDN based network intrusion detection system using machine learning approaches," Peer-to-Peer Netw. Appl., vol. 12, no. 2, pp. 493-501, 2019.

[11] J. Xie et al., "A survey of machine learning techniques applied to software defined networking (SDN): Research issues and challenges," IEEE Commun. Surv. Tutorials, vol. 21, no. 1, pp. 393-430, 2019.

[12] J. Singh and S. Behal, "Detection and mitigation of DDoS attacks in SDN: A comprehensive review, research challenges and future directions," Comput. Sci. Rev., vol. 37, p. 100279, 2020.

[13] T. Jafarian, M. Masdari, A. Ghaffari, and K. Majidzadeh, "A survey and classification of the security anomaly detection mechanisms in software defined networks," Cluster Comput., vol. 1, 2020.

[14] Y. Hande and A. Muddana, "A survey on intrusion detection system for software defined networks (SDN)," Int. J. Bus. Data Commun. Netw., vol. 16, no. 1, pp. 28-47, 2020.

[15] L. F. Eliyan and R. Di Pietro, "DoS and DDoS attacks in Software Defined Networks: A survey of existing solutions and research challenges," Futur. Gener. Comput. Syst., vol. 122, pp. 149-171, 2021.

[16] Y. Cui et al., "Towards DDoS detection mechanisms in SoftwareDefined Networking," J. Netw. Comput. Appl., vol. 190, no. July, p. 103156, 2021.

[17] "Open Networking Foundation. 2021. Software-Defined Networking (SDN) Definition - Open Networking Foundation. [online]," Open Networking Foundation, 2013. [Online]. Available: https://searchnetworking.techtarget.com/definition/software-definednetworking-SDN. [Accessed: 08-Feb-2021].

[18] W. Braun and M. Menth, "Software-Defined Networking Using OpenFlow: Protocols, Applications and Architectural Design Choices," Futur. Internet, vol. 6, no. 2, pp. 302-336, 2014.

[19] “Open vSwitch." [Online]. Available: https://www.openvswitch.org/. [Accessed: 07-Nov-2021].

[20] "Pantou - OpenFlow 1.3 for OpenWRT." [Online]. Available: https://github.com/CPqD/openflow-openwrt. [Accessed: 07-Nov2021].

[21] "Indigo - Core OpenFlow agent and infrastructure modules." [Online]. Available:

https://floodlight.atlassian.net/wiki/spaces/Indigo/overview. 
[Accessed: 07-Nov-2021]

[22] A. Voellmy and P. Hudak, "Nettle: Functional Reactive Programming of OpenFlow Networks," in YALE UNIV NEW HAVEN CT DEPT OF COMPUTER SCIENCE, 2010.

[23] J. W. Lockwood et al., "NetFPGA-an open platform for gigabit-rate network switching and routing," in IEEE International Conference on Microelectronic Systems Education (MSE’07), 2007, p. 177.

[24] G. Lu et al., "Serverswitch: a programmable and high performance platform for data center networks," USENIX Symp. Networked Syst. Des. Implement., vol. 11, pp. 1-14, 2011.

[25] M. B. Anwer, M. Motiwala, M. Bin Tariq, and N. Feamster, "SwitchBlade: A platform for rapid deployment of network protocols on programmable hardware," in Proceedings of the ACM SIGCOMM 2010 conference, pp. 183-194.

[26] A. Ben Letaifa, SSIM and ML based QoE enhancement approach in SDN context, 1st ed., vol. 114. Elsevier Inc., 2019.

[27] S. Gao, Z. Li, B. Xiao, and G. Wei, "Security Threats in the Data Plane of Software-Defined Networks," IEEE Netw., vol. 32, no. 4, pp. 108 113,2018

[28] D. B. Rawat and S. R. Reddy, "Software defined networking architecture, security and energy efficiency: A survey," Softw. Defin. Netw. Archit. Secur. Energy Effic. A Surv., vol. 19, no. 1, pp. 325346, 2017.

[29] "RYU Controller." [Online]. Available: https://ryu-sdn.org/. [Accessed: 25-Dec-2020].

[30] L. R. Prete, A. A. Shinoda, C. M. Schweitzer, and R. L. S. De Oliveira, "Simulation in an SDN network scenario using the POX Controller," in 2014 IEEE Colombian Conference on Communications and Computing, COLCOM 2014 - Conference Proceedings, 2014, no. June, pp. 1-6.

[31] N. Gude et al., "NOX: towards an operating system for networks," ACM SIGCOMM Comput. Commun. Rev., vol. 38, no. 3, pp. 105110, 2008.

[32] "Opendaylight Controller." [Online]. Available: https://www.opendaylight.org/about/platform-overview. [Accessed: 28-Dec-2020].

[33] FloodLight Controller, "FloodLight Controller." [Online]. Available: https://floodlight.atlassian.net/wiki/spaces/floodlightcontroller/overvi ew. [Accessed: 27-Dec-2020].

[34] "ONOS Controller." [Online]. Available: https://opennetworking.org/onos/. [Accessed: 27-Dec-2020].

[35] A. Dawoud, S. Shahristani, and C. Raun, "Deep learning and softwaredefined networks: Towards secure IoT architecture," Internet of Things, vol. 3-4, pp. 82-89, 2018.

[36] A. Yazdinejadna, R. M. Parizi, A. Dehghantanha, and M. S. Khan, "A kangaroo-based intrusion detection system on software-defined networks," Comput. Networks, vol. 184, p. 107688, 2021.

[37] P. Dong, X. Du, H. Zhang, and T. Xu, "A detection method for a novel DDoS attack against SDN controllers by vast new low-traffic flows," in 2016 IEEE International Conference on Communications, ICC 2016, 2016.

[38] "OpenFlow Switch Specification Version 1.3.5 ( Protocol version 0x04 )," 2015. [Online]. Available: https://opennetworking.org/wpcontent/uploads/2014/10/openflow-switch-v1.3.5.pdf. [Accessed: 19Dec-2021].

[39] “'Openflow - The Workflow Introduction'. SDN For You, 2020," Openflow - The Workflow Introduction, 2015. [Online]. Available: https://sdnforyou.wordpress.com/2015/08/12/openflow-the-

workflow-introduction/. [Accessed: 19-Nov-2020].

[40] A. Bahnasse, F. E. Louhab, H. Ait Oulahyane, M. Talea, and A. Bakali, "Novel SDN architecture for smart MPLS Traffic EngineeringDiffServ Aware management," Futur. Gener. Comput. Syst., vol. 87, pp. 115-126, 2018.

[41] S. Agarwal, K. Murali, and L. T.V, "Traffic Engineering in Software Defined Networks," in Proceedings IEEE INFOCOM, 2013, pp. 22112219.

[42] D. M. F. Mattos et al., "OMNI: OpenFlow MaNagement Infrastructure,” 2011 Int. Conf. Netw. Futur. NOF 2011 - Proc., pp. 52 56, 2011.

[43] Y. Minlam, J. Lavanya, and M. Rui, "Software Defined Traffic Measurement with OpenSketch," in USENIX Symposium on Networked Systems Design and Implementation (NSDI '13), 2013, pp. 29-42.

[44] R. Van Der Pol et al., "Multipathing with MPTCP and open flow," in Proceedings - 2012 SC Companion: High Performance Computing,
Networking Storage and Analysis, SCC 2012, 2012, pp. 1617-1624. R. Trestian, G. M. Muntean, and K. Katrinis, "MiceTrap: Scalable traffic engineering of datacenter mice flows using OpenFlow," Proc. 2013 IFIP/IEEE Int. Symp. Integr. Netw. Manag. IM 2013, pp. 904907, 2013.

[46] R. Bennesby, P. Fonseca, E. Mota, and A. Passito, "An inter-AS routing component for software-defined networks," Proc. 2012 IEEE Netw. Oper. Manag. Symp. NOMS 2012, pp. 138-145, 2012.

[47] H. Zhang and J. Yan, "Performance of SDN Routing in Comparison with Legacy Routing Protocols," Proc. - 2015 Int. Conf. CyberEnabled Distrib. Comput. Knowl. Discov. CyberC 2015, pp. 491-494, 2015.

[48] R. R. Fontes, S. Afzal, S. H. B. Brito, M. A. S. Santos, and C. E. Rothenberg, "Mininet-WiFi: Emulating software-defined wireless networks," Proc. 11th Int. Conf. Netw. Serv. Manag. CNSM 2015, pp. 384-389, 2015.

[49] P. Jakma and D. Lamparter, "Introduction to the Quagga Routing Suite," IEEE Netw., vol. 28, no. 2, pp. 42-48, 2014.

[50] M. Hata, M. Soylu, S. Izumi, T. Abe, and T. Suganuma, "SDN based end-to-end inter-domain routing mechanism for mobility management and its evaluation," Sensors (Switzerland), vol. 18, no. 12, 2018.

[51] S. A. Mehdi, J. Khalid, and S. A. Khayam, "Revisiting Traffic Anomaly Detection Using Software Defined Networking," in RAID 2011: Recent Advances in Intrusion Detection, 2011, pp. 161-180.

[52] N. Ankur, R. Alex, F. Nick, and C. Russ, "Resonance: Dynamic Access Control for Enterprise Networks," in WREN '09: Proceedings of the 1st ACM workshop on Research on enterprise networking, 2009, no. 9783662530924, pp. 11-18.

[53] C. Yu Hunag, T. Min Chi, C. Yao Ting, C. Yu Chieh, and C. Yan Ren, "A novel design for future on-demand service and security," Int. Conf. Commun. Technol. Proceedings, ICCT, pp. 385-388, 2010.

[54] N. . M. Chowdhury and R. Boutaba, "A Survey of Network Virtualization," Comput. Networks, vol. 54, no. 8, pp. 862-876, 2010. Q. Duan, N. Ansari, and M. Toy, "Software-defined network virtualization: An architectural framework for integrating SDN and NFV for service provisioning in future networks," IEEE Netw., vol. 30, no. 5, pp. 10-16, 2016.

[56] A. D. Ferguson, A. Guha, C. Liang, R. Fonseca, and S. Krishnamurthi, "Hierarchical policies for software defined networks," HotSDN'12 Proc. 1st ACM Int. Work. Hot Top. Softw. Defin. Networks, pp. 3742, 2012.

[57] P. Manso and J. Moura, "SDN-Based Intrusion Detection System for Early Detection and Mitigation of DDoS Attacks," Inf., vol. 10, no. 3, pp. 1-17, 2019.

[58] A. Garg and P. Maheshwari, "A Hybrid Intrusion Detection System: A Review," in International Conference on (ISCO) Intelligent Systems and Control, 2016, pp. 1-5.

[59] L. T. Heberlein, G. V. Dias, K. N. Levitt, B. Mukherjee, J. Wood, and D. D. Wolber, "A network security monitor," in Proceedings of the Symposium on Security and Privacy, 1990, pp. 296-304.

[60] Q. Yan, F. R. Yu, Q. Gong, and J. Li, "Software-defined networking (SDN) and distributed denial of service (DDOS) attacks in cloud computing environments: A survey, some research issues, and challenges," IEEE Commun. Surv. Tutorials, vol. 18, no. 1, pp. $602-$ 622, 2016.

[61] S. Madhawa, P. Balakrishnan, and U. Arumugam, "Data driven intrusion detection system for software defined networking enabled industrial internet of things," J. Intell. Fuzzy Syst., vol. 34, no. 3, pp. 1289-1300, 2018.

[62] P. Wang, L. T. Yang, X. Nie, Z. Ren, J. Li, and L. Kuang, "Data-driven software defined network attack detection: State-of-the-art and perspectives," Inf. Sci. (Ny)., vol. 513, no. xxxx, pp. 65-83, 2020.

[63] P. García-Teodoro, J. Díaz-Verdejo, G. Maciá-Fernández, and E. Vázquez, "Anomaly-based network intrusion detection: Techniques, systems and challenges," Comput. Secur., vol. 28, no. 1-2, pp. 18-28, 2009.

[64] B. Mantur, A. Desai, and K. S. Nagegowda, "Centralized Control Signature-Based Firewall and Statistical-Based Network Intrusion Detection System (NIDS) in Software Defined Networks (SDN)," in Emerging Research in Computing, Information, Communication and Applications, 2015.

[65] S. M. Mousavi and M. St-Hilaire, "Early detection of DDoS attacks against SDN controllers," in nternational Conference on Computing, Networking and Communications, ICNC, 2015, pp. 77-81.

[66] R. Sahay et al., "Towards autonomic DDoS mitigation using Software 
Defined Networking," in SENT 2015: NDSS workshop on security of emerging networking technologies. Internet society, 2015.

[67] S. Jevtic, H. Lotfalizadeh, and D. S. Kim, "Toward network-based DDoS detection in software-defined networks," in 12th International Conference on Ubiquitous Information Management and Communication, 2018.

[68] N. Z. Bawany, J. A. Shamsi, and K. Salah, "DDoS Attack Detection and Mitigation Using SDN: Methods, Practices, and Solutions," Arab. J. Sci. Eng., vol. 42, no. 2, pp. 425-441, 2017.

[69] S. Shin, P. Porras, V. Yegneswaran, M. Fong, G. Gu, and M. Tyson, "Fresco: Modular composable security services for software-defined networks," in 20th Annual Network \& Distributed System Security Symposium. Ndss, 2013, no. February.

[70] R. Jin and B. Wang, "Malware detection for mobile devices using software-defined networking," in Proceedings - 2013 2nd GENI Research and Educational Experiment Workshop, GREE, 2013, pp. 81-88.

[71] M. Zuzčák and M. Zenka, "Expert system assessing threat level of attacks on a hybrid SSH honeynet," Comput. Secur., vol. 92, p. 101784, 2020.

[72] A. K. Ghosh, A. Schwartzbard, and M. Schatz, "Learning program behavior profiles for intrusion detection," in Proceedings of the Workshop on Intrusion Detection and Network Monitoring, 1999, pp. $1-13$.

[73] Q. Niyaz, W. Sun, A. Y. Javaid, and M. Alam, "A deep learning approach for network intrusion detection system," in Eai Endorsed Transactions on Security and Safety, 2016, vol. 3, no. 9.

[74] A. Le, P. Dinh, H. Le, and N. C. Tran, "Flexible Network-Based Intrusion Detection and Prevention System on Software-Defined Networks," in Proceedings - 2015 International Conference on Advanced Computing and Applications, ACOMP 2015, 2016, pp. $106-111$.

[75] Y. J. Chew, S. Y. Ooi, K.-S. Wong, and Y. H. Pang, "Decision Tree with Sensitive Pruning in Network-based Intrusion Detection System," in Computational Science and Technology. Lecture Notes in Electrical Engineering, 2020, vol. 603, no. August, pp. 425-433.

[76] N. Thapa, Z. Liu, D. B. Kc, B. Gokaraju, and K. Roy, "Comparison of machine learning and deep learning models for network intrusion detection systems," Futur. Internet, vol. 12, no. 10, pp. 1-16, 2020.

[77] N. Chaabouni, M. Mosbah, A. Zemmari, C. Sauvignac, and P. Faruki, "Network Intrusion Detection for IoT Security Based on Learning Techniques," IEEE Commun. Surv. Tutorials, vol. 21, no. 3, pp. 26712701, 2019.

[78] L. Nicholas, S. Y. Ooi, Y. H. Pang, S. O. Hwang, and S.-Y. Tan, "Study of long short-term memory in flow-based network intrusion detection system,” J. Intell. Fuzzy Syst., vol. 35, no. 6, pp. 5947-5957, 2018.

[79] A. R. Mohammed, S. A. Mohammed, and S. Shirmohammadi, "Machine Learning and Deep Learning Based Traffic Classification and Prediction in Software Defined Networking," in 2019 IEEE International Symposium on Measurements and Networking, $\mathrm{M}$ and $\mathrm{N}$ 2019 - Proceedings, 2019.

[80] Y. Zeng, H. Gu, W. Wei, and Y. Guo, "Deep-Full-Range: A Deep Learning Based Network Encrypted Traffic Classification and Intrusion Detection Framework," IEEE Access, vol. 7, pp. 4518245190, 2019.

[81] C. Zhang et al., "A Novel Framework Design of Network Intrusion Detection Based on Machine Learning Techniques," Secur. Commun. Networks, vol. 2021, 2021.

[82] P. Lichodzijewski, A. N. Zincir-Heywood, and M. I. Heywood, "Hostbased intrusion detection using self-organizing maps," in Proceedings of the International Joint Conference on Neural Networks, 2002, vol. 2, pp. 1714-1719.

[83] I. Khalkhali, R. Azmi, M. Azimpour-kivi, and M. Khansari, "Hostbased Web Anomaly Intrusion Detection System , an Artificial Immune System Approach,” J. Comput. Sci., vol. 8, no. 5, pp. 14-24, 2011.

[84] E. W. and B. Y. Saurav Nanda, Faheem Zafari, Casimer DeCusatis, "Predicting Network Attack Patterns in SDN using Machine Learning Approach," in IEEE Conference on Network Function Virtualization and Software Defined Networks (NFV-SDN), 2016, pp. 261-266.

[85] G. Serpen and E. Aghaei, "Host-based misuse intrusion detection using PCA feature extraction and kNN classification algorithms," Intell. Data Anal., vol. 22, no. 5, pp. 1101-1114, 2018.

[86] M. Nobakht, V. Sivaraman, and R. Boreli, "A host-based intrusion detection and mitigation framework for smart home IoT using
OpenFlow," in 11th International Conference on Availability, Reliability and Security, ARES, 2016, pp. 147-156.

[87] M. Xie and J. Hu, "Evaluating host-based anomaly detection systems: A preliminary analysis of ADFA-LD," in Proceedings of the 2013 6th International Congress on Image and Signal Processing, CISP 2013, 2013, vol. 3, no. Cisp, pp. 1711-1716.

[88] D. Y. Yeung and Y. Ding, "Host-based intrusion detection using dynamic and static behavioral models," Pattern Recognit., vol. 36, no. 1, pp. 229-243, 2003.

[89] X. Xu and T. Xie, "A reinforcement learning approach for host-based intrusion detection using sequences of system calls," Adv. Intell. Comput. ICIC 2005. Lect. Notes Comput. Sci., vol. 3644 LNCS, pp. 995-1003, 2005

[90] A. Chawla, B. Lee, S. Fallon, and P. Jacob, "Host Based Intrusion Detection System with Combined CNN/RNN Model," in ECML PKDD 2018 Workshops. ECML PKDD 2018. Lecture Notes in Computer Science, 2019, vol. 11329 LNAI, pp. 149-158.

[91] H. Y. Gyuwan Kim, J. Lee, Y. Paek, and S. Yoon, "LSTM-based system-call language modeling and robust ensemble method for designing host-based intrusion detection system," arXiv:1611.01726v1, pp. 1-12, 2016.

[92] D. H. Lee, D. Y. Kim, and J. Il Jung, "Multi-stage intrusion detection system using hidden Markov model algorithm," in Proceedings of the International Conference on Information Science and Security, ICISS 2008, 2008, pp. 72-77.

[93] T. M. Chen and P. J. Walsh, "Guarding Against Network Intrusions," in Network and System Security: Second Edition, 2014, pp. 57-82.

[94] M. A. Aydin, A. H. Zaim, and K. G. Ceylan, "A hybrid intrusion detection system design for computer network security," Comput. Electr. Eng., vol. 35, no. 3, pp. 517-526, 2009.

[95] T. Ha et al., "Suspicious traffic sampling for intrusion detection in software-defined networks," Comput. Networks, vol. 109, pp. 172$182,2016$.

[96] O. Depren, M. Topallar, E. Anarim, and M. K. Ciliz, “An intelligent intrusion detection system (IDS) for anomaly and misuse detection in computer networks," Expert Syst. Appl., vol. 29, no. 4, pp. 713-722, 2005.

[97] K. Giotis, G. Androulidakis, and V. Maglaris, "Leveraging SDN for efficient anomaly detection and mitigation on legacy networks," Proc. - 2014 3rd Eur. Work. Software-Defined Networks, EWSDN 2014, no. i, pp. 85-90, 2014.

[98] M. T. Ali A. Ghorbani, Wei Lu, "Network Intrusion Detection and Prevention Advances in Information Security," vol. 47, 2010.

[99] F. Ali and B. Hamid, "Development of Host Based Intrusion Detection System for Log Files," in IEEE Symposium on Business, Engineering and Industrial Applications (ISBEIA), 2011, pp. 281-285.

[100] Y. Lin, Y. Zhang, and Y. J. Ou, "The design and implementation of host-based intrusion detection system," 3rd Int. Symp. Intell. Inf. Technol. Secur. Informatics, IITSI 2010, pp. 595-598, 2010.

[101] Y. Yu, J. Long, and Z. Cai, "Session-based network intrusion detection using a deep learning architecture," in International Conference on Modeling Decisions for Artificial Intelligence, 2017, no. October, pp. 144-155.

[102] S. Ponomarev and T. Atkison, "Session Duration Based Feature Extraction for Network Intrusion Detection in Control System Networks," in Proceedings - 2016 International Conference on Computational Science and Computational Intelligence, CSCI 2016, 2017, pp. 892-896.

[103] B. D. Caulkin, J. Lee, and M. Wang, "Packet- vs. session-based modeling for intrusion detection systems," in International Conference on Information Technology: Coding and Computing, ITCC, 2005, vol. 1, pp. 116-121.

[104] M. F. Umer, M. Sher, and Y. Bi, "Flow-based intrusion detection: Techniques and challenges," Comput. Secur., vol. 70, pp. 238-254, 2017.

[105] A. Qayyum, M. H. Islam, and M. Jamil, "Taxonomy of statistical based anomaly detection techniques for intrusion detection," in Proceedings - IEEE 2005 International Conference on Emerging Technologies, ICET 2005, 2005, vol. 2005, pp. 270-276.

[106] Bavani K, R. M. P, and E. S. G. S. R, "Statistical Approach Based Detection of Distributed Denial of Service Attack in a Software Defined Network," in 6th International Conference on Advanced Computing \& Communication Systems (ICACCS), 2020.

[107] A. Sperotto, R. Sadre, and A. Pras, "Anomaly characterization in flowbased traffic time series," in IP Operations and Management. IPOM 
2008. Lecture Notes in Computer Science, 2008, vol. 5275 LNCS, pp. $15-27$.

[108] G. Fernandes, J. J. P. C. Rodrigues, and M. L. Proença, “Autonomous profile-based anomaly detection system using principal component analysis and flow analysis," Appl. Soft Comput. J., vol. 34, pp. 513$525,2015$.

[109] C. Zhang, Z. Cai, W. Chen, X. Luo, and J. Yin, "Flow level detection and filtering of low-rate DDoS," Comput. Networks, vol. 56, no. 15, pp. 3417-3431, 2012.

[110] H. A. Nguyen, T. Van Nguyen, D. Il Kim, and D. Choi, "Network Traffic Anomalies Detection and Identification with Flow Monitoring," in 5th IFIP International Conference on Wireless and Optical Communications Networks (WOCN'08), 2008.

[111] T. Naqash, S. H. Shah, and M. N. U. Islam, "Statistical Analysis Based Intrusion Detection System for Ultra-High-Speed Software Defined Network,” Int. J. Parallel Program., vol. 0, pp. 1-26, 2021.

[112] L. Boero, M. Marchese, and S. Zappatore, "Support Vector Machine Meets Software Defined Networking in IDS Domain," Proc. 29th Int. Teletraffic Congr. ITC 2017, vol. 3, pp. 25-30, 2017.

[113] G. Li, M. Dong, K. Ota, J. Wu, J. Li, and T. Ye, "Deep Packet Inspection based Application-Aware Traffic Control for Software Defined Networks," in IEEE Global Communications Conference (GLOBECOM), 2016

[114] G. Schaffrath and B. Stiller, "Conceptual integration of flow-based and packet-based network intrusion detection," in FIP International Conference on Autonomous Infrastructure, Management and Security, 2008, vol. 5127 LNCS, pp. 190-194.

[115] B. Andreas, J. Dilruksha, E. McCandless, S. Chakrabarty, and O. Youssef, "Flow-Based and Packet-Based Intrusion Detection Using BLSTM," SMU Data Sci. Rev., vol. 3, no. 3, 2020.

[116] Q. Dang, "Studying machine learning techniques for intrusion detection systems," in International Conference on Future Data and Security Engineering, 2019, pp. 411-426.

[117] Willi Richert and Luis Pedro Coelho, Building Machine Learning Systems with Python. 2013.

[118] K. S.B., "Supervised Machine Learning: A Review of Classification Techniques," Informatica, vol. 31, pp. 249-268, 2007.

[119] Lowd Daniel and D. Pedro, "Naive Bayes models for probability estimation.pdf," in International Conference on Machine Learning, 2005, pp. 529-536.

[120] P.-Y. Zhou and K. C. C. Chan, "A Model-Based Multivariate Time Series Clustering Algorithm Pei-Yuan," in PAKDD 2014: Trends and Applications in Knowledge Discovery and Data Mining, 2014, pp. 805-817.

[121] Q. Cheng et al., "Machine learning based malicious payload identification in software-defined networking," J. Netw. Comput. Appl., vol. 192, p. 103186, 2021.

[122] C. Yu, J. Lan, J. C. Xie, and Y. Hu, "QoS-aware traffic classification architecture using machine learning and deep packet inspection in SDNs," Procedia Comput. Sci., vol. 131, pp. 1209-1216, 2018.

[123] Z. Zhou and M. Li, "Tri-Training: Exploiting Unlabeled Data Using Three Classifiers," IEEE Trans. Knowl. Data Eng., vol. 17, no. 11, pp. 1529-1541, 2005.

[124] Y. D. Lin, P. C. Lin, C. H. Yeh, Y. C. Wang, and Y. C. Lai, “An extended SDN architecture for network function virtualization with a case study on intrusion prevention," IEEE Netw., vol. 29, no. 3, pp. $48-53,2015$.

[125] T. C. Jr and X. Mountrouidou, "Selective Packet Inspection to Detect DoS Flooding Using Software Defined Networking ( SDN )," in IEEE 35th international conference on distributed computing systems workshops., 2015.

[126] K. Cabaj, M. Gregorczyk, and W. Mazurczyk, "Software-defined networking-based crypto ransomware detection using HTTP traffic characteristics," Comput. Electr. Eng., vol. 66, pp. 353-368, 2018.

[127] G. Cusack, O. Michel, and E. Keller, "Machine learning-based detection of ransomware using SDN," SDN-NFVSec 2018 - Proc. 2018 ACM Int. Work. Secur. Softw. Defin. Networks Netw. Funct. Virtualization, Co-located with CODASPY 2018, vol. 2018-Janua, pp. $1-6,2018$.

[128] C. Song, Y. Park, K. Golani, Y. Kim, K. Bhatt, and K. Goswami, "Machine-learning based threat-aware system in software defined networks," 2017 26th Int. Conf. Comput. Commun. Networks, ICCCN 2017, 2017.

[129] A. Santos Da Silva, J. A. Wickboldt, L. Z. Granville, and A. SchaefferFilho, "ATLANTIC: A framework for anomaly traffic detection, classification, and mitigation in SDN," Proc. NOMS 2016 - 2016 IEEE/IFIP Netw. Oper. Manag. Symp., no. Noms, pp. 27-35, 2016.

[130] H. Peng, Z. Sun, X. Zhao, S. Tan, and Z. Sun, "A Detection Method for Anomaly Flow in Software Defined Network," IEEE Access, vol. 6, pp. 27809-27817, 2018.

[131] N. Satheesh et al., "Flow-based anomaly intrusion detection using machine learning model with software defined networking for OpenFlow network," Microprocess. Microsyst., vol. 79, p. 103285, 2020.

[132] A. Abubakar and B. Pranggono, "Machine Learning Based Intrusion Detection System for Software Defined Networks," Int. Conf. Emerg. Secur. Technol., pp. 138-143, 2015.

[133] Q. Schueller, K. Basu, M. Younas, M. Patel, and F. Ball, "A Hierarchical Intrusion Detection System using Data Center," in 2018 28th International Telecommunication Networks and Applications Conference (ITNAC), 2018, pp. 1-6.

[134] G. A. Ajaeiya, N. Adalian, I. H. Elhajj, A. Kayssi, and A. Chehab, "Flow-based Intrusion Detection System for SDN," in Proceedings IEEE Symposium on Computers and Communications, 2017, pp. 787793.

[135] P. Wang, K. M. Chao, H. C. Lin, W. H. Lin, and C. C. Lo, “An Efficient Flow Control Approach for SDN-Based Network Threat Detection and Migration Using Support Vector Machine," Proc. - 13th IEEE Int. Conf. E-bus. Eng. ICEBE 2016 - Incl. 12th Work. Serv. Appl. Integr. Collab. SOAIC 2016, pp. 56-63, 2017

[136] L. Yang and H. Zhao, "DDoS attack identification and defense using SDN based on machine learning method," in Proceedings - 2018 15th International Symposium on Pervasive Systems, Algorithms and Networks, I-SPAN 2018, 2019, pp. 174-178.

[137] A. Alshamrani, A. Chowdhary, S. Pisharody, D. Lu, and D. Huang, "A defense system for defeating DDoS attacks in SDN based networks," in MobiWac 2017 - Proceedings of the 15th ACM International Symposium on Mobility Management and Wireless Access, Colocated with MSWiM 2017, 2017, pp. 83-92.

[138] M. A. Hall, "Correlation-based Feature Selection for Machine Learning," 1999.

[139] V. Vetriselvi, P. S. Shruti, and S. Abraham, "Two-level intrusion detection system in SDN using machine learning," in Proceedings of the International Conference on Communications and Cyber Physical Engineering, 2018, pp. 449-461.

[140] X. S. Yang and A. H. Gandomi, "Bat algorithm: A novel approach for global engineering optimization," Eng. Comput. (Swansea, Wales), vol. 29 , no. 5, pp. 464-483, 2012.

[141] J. Li, Z. Zhao, R. Li, and H. Zhang, "AI-based two-stage intrusion detection for software defined IoT networks," IEEE Internet Things J., vol. 6, no. 2, pp. 2093-2102, 2019.

[142] A. M. Fatih, G. Cengiz, and K. Enis, "Usage of Machine Learning Algorithms for Flow Based Anomaly Detection System in Software Defined Networks," in Intelligent and Fuzzy Techniques: Smart and Ennovative Solutions, 2021, pp. 1156-1163.

[143] M. S. Elsayed, N. A. Le-Khac, S. Dev, and A. D. Jurcut, "MachineLearning Techniques for Detecting Attacks in SDN," in Proceedings of IEEE 7th International Conference on Computer Science and Network Technology, ICCSNT 2019, 2019, pp. 277-281.

[144] N. N. Tuan, P. H. Hung, N. D. Nghia, N. Van Tho, T. Van Phan, and N. H. Thanh, "A DDoS attack mitigation scheme in ISP networks using machine learning based on SDN," Electron., vol. 9, no. 3, pp. 1-19, 2020.

[145] "Goldstein, M., 2021. Markus-Go/bonesi. [online] GitHub. Available at: <https://github.com/Markus-Go/bonesi> [Accessed 15 February 2021].".

[146] A. Banitalebi Dehkordi, M. R. Soltanaghaei, and F. Z. Boroujeni, "The DDoS attacks detection through machine learning and statistical methods in SDN," J. Supercomput., vol. 77, no. 3, pp. 2383-2415, 2020.

[147] J. A. Perez-Diaz, I. A. Valdovinos, K. K. R. Choo, and D. Zhu, “A Flexible SDN-Based Architecture for Identifying and Mitigating LowRate DDoS Attacks Using Machine Learning," IEEE Access, vol. 8, pp. 155859-155872, 2020.

[148] A. Sebbar, K. Zkik, Y. Baddi, M. Boulmalf, and M. D. E. C. El Kettani, "MitM detection and defense mechanism CBNA-RF based on machine learning for large-scale SDN context," J. Ambient Intell. Humaniz. Comput., vol. 11, no. 12, pp. 5875-5894, 2020.

[149] J. Aiken and S.-H. Sandra, "Investigating Adversarial Attacks against Network Intrusion Detection Systems in SDNs," in IEEE Conference 
on Network Functions Virtualization and Software Defined Networks, 2019, pp. 1-7.

[150] S. Lee, J. Kim, S. Shin, P. Porras, and V. Yegneswaran, "Athena: A Framework for Scalable Anomaly Detection in Software-Defined Networks," in Proceedings - 47th Annual IEEE/IFIP International Conference on Dependable Systems and Networks, DSN 2017, 2017, pp. 249-260.

[151] N. Meti, D. G. Narayan, and V. P. Baligar, "Detection of distributed denial of service attacks using machine learning algorithms in software defined networks," in 2017 International Conference on Advances in Computing, Communications and Informatics, ICACCI, pp. 13661371.

[152] R. Santos, D. Souza, W. Santo, A. Ribeiro, and E. Moreno, "Machine learning algorithms to detect DDoS attacks in SDN," Concurr. Comput. Pract. Exp., vol. 32, no. 16, pp. 1-14, 2020.

[153] P. Hadem, D. K. Saikia, and S. Moulik, "An SDN-based Intrusion Detection System using SVM with Selective Logging for IP Traceback," Comput. Networks, vol. 191, no. September 2020, p. 108015, 2021.

[154] D. Hu, P. Hong, and Y. Chen, "FADM : DDoS Flooding Attack Detection and Mitigation System in Software-Defined Networking," in GLOBECOM 2017-2017 IEEE Global Communications Conference., 2017, pp. 1-7.

[155] H. Polat and O. Polat, "Detecting DDoS Attacks in Software-Defined Networks Through Feature Selection Methods and Machine Learning Models," sustainability, vol. 12, no. 3,1035, 2020.

[156] T. Kohonen, "Essentials of the self-organizing map," Neural Networks, vol. 37, pp. 52-65, 2013.

[157] “CTU-BOTNET." [Online]. Available: https://mcfp.weebly.com/. [Accessed: 20-Oct-2021].

[158] "Malware Traffic Analysis," Malware-Traffic-Analysis.net, 2018 [Online]. Available: https://www.malware-traffic-analysis.net/. [Accessed: 21-Jan-2021].

[159] G. Sun, W. Jiang, Y. Gu, D. Ren, and H. Li, "DDoS Attacks and Flash Event Detection Based on Flow Characteristics in SDN," in Proceedings of AVSS 2018-15th IEEE International Conference on Advanced Video and Signal-Based Surveillance, 2018, pp. 1-6.

[160] P. Phaal, S. Panchen, and N. McKee, "Traffic Monitoring using sFlow," RFC3176 InMon Corp. sFlow A Method Monit. Traffic Switch. Routed Networks, 2001.

[161] L. Van Der Maaten, "Accelerating t-SNE using tree-based algorithms," J. Mach. Learn. Res., vol. 15, pp. 3221-3245, 2015.

[162] "Faucet controller." [Online]. Available: https://faucet.nz/. [Accessed: 20-Feb-2021].

[163] M. M. Oo, S. Kamolphiwong, and T. Kamolphiwong, "The Design of SDN Based Detection for Distributed Denial of Service (DDoS) Attack," ICSEC - 21st Int. Comput. Sci. Eng. Conf., vol. 6, pp. 258263, 2018.

[164] T. M. Nam et al., "Self-organizing map-based approaches in DDoS flooding detection using SDN," in International Conference on Information Networking, 2018, pp. 249-254.

[165] M. Almi, A. A. Ghazleh, A. Al-rahayfeh, and A. Razaque, "Intelligent intrusion detection system using clustered self organized map," in International Conference on Software Defined Systems, 2018, no. 1, pp. $138-144$.

[166] H. Gunes Kayacik, A. Nur Zincir-Heywood, and M. I. Heywood, “A hierarchical SOM-based intrusion detection system," Eng. Appl. Artif. Intell., vol. 20, no. 4, pp. 439-451, 2007.

[167] T. Hurley, J. E. Perdomo, and A. Perez-Pons, "HMM-based intrusion detection system for software defined networking," in 15th IEEE International Conference on Machine Learning and Applications, ICMLA 2016, 2017, pp. 617-621.

[168] T. Guelzim and M. S. Obaidat, Formal methods of attack modeling and detection. Elsevier Inc., 2015.

[169] W. Wang, X. Ke, and L. Wang, "A HMM-R approach to detect LDDoS attack adaptively on SDN controller," Futur. Internet, vol. 10, no. $9,2018$.

[170] R. Braga, E. Mota, and A. Passito, "Lightweight DDoS flooding attack detection using NOX/OpenFlow," in Proceedings - 35th Annual IEEE Conference on Local Computer Networks, LCN, 2010, pp. 408-415.

[171] L. Barki, A. Shidling, N. Meti, D. G. Narayan, and M. M. Mulla, "Detection of distributed denial of service attacks in software defined networks," in 2016 International Conference on Advances in Computing, Communications and Informatics, ICACCI 2016, 2016, pp. 2576-2581.
D. Jankowski and M. Amanowicz, "On Efficiency of Selected Machine Learning Algorithms for Intrusion Detection in Software Defined Networks," Int. J. Electron. Telecommun., vol. 62, no. 3, pp. 247-252, 2016.

[173] D. E. Moriarty, A. C. Schultz, and J. J. Grefenstette, "Evolutionary Algorithms for Reinforcement Learning," J. Artif. Intell. Res., vol. 11, pp. 241-276, 1999

[174] C. J. C. H. WATKINS and P. DAYA, "Q-Learning," Mach. Learn., vol. 8, pp. 279-292, 2020.

[175] V. Mnih et al., "Human-level control through deep reinforcement learning," Nature, vol. 518, no. 7540, pp. 529-533, 2015.

[176] J. Schulman, F. Wolski, P. Dhariwal, A. Radford, and O. Klimov, "Proximal Policy Optimization Algorithms," arXiv:1707.06347v2 [cs.LG], pp. 1-12, 2017.

[177] C. C.-Y. Hsu, C. Mendler-Dünner, and M. Hardt, "Revisiting Design Choices in Proximal Policy Optimization," arXiv:2009.10897v1 [cs.LG], 2020.

[178] L. S. R. Sampaio, P. H. A. Faustini, A. S. Silva, L. Z. Granville, and A. Schaeffer-Filho, "Using NFV and Reinforcement Learning for Anomalies Detection and Mitigation in SDN," in Proceedings - IEEE Symposium on Computers and Communications, 2018, pp. 432-437.

[179] M. Zolotukhin, S. Kumar, and T. Hamalainen, "Reinforcement learning for attack mitigation in SDN-enabled networks," in Proceedings of the 2020 IEEE Conference on Network Softwarization: Bridging the Gap Between AI and Network Softwarization, NetSoft 2020, 2020, pp. 282-286.

[180] I. Akbari, E. Tahoun, M. A. Salahuddin, N. Limam, and R. Boutaba, "ATMoS: Autonomous Threat Mitigation in SDN using Reinforcement Learning," in Proceedings of IEEE/IFIP Network Operations and Management Symposium 2020: Management in the Age of Softwarization and Artificial Intelligence, NOMS 2020, 2020, pp. $1-9$.

[181] T. V Phan, T. M. R. Gias, and S. T. Islam, "Q-MIND: Defeating Stealthy DoS Attacks in SDN with a Machine-learning based Defense Framework," in IEEE Global Communications Conference (GLOBECOM), 2019, pp. 1-6.

[182] P. T. Dinh and M. Park, "BDF-SDN: A Big Data Framework for DDoS Attack Detection in Large-Scale SDN-Based Cloud," in IEEE Conference on Dependable and Secure Computing, DSC, 2021, pp. 18 .

[183] P. Wette, A. Schwabe, F. Wallaschek, M. H. Zahraee, and H. Karl, "MaxiNet: Distributed Emulation of Software-Defined Networks," in IFIP Networking Conference. IEEE, 2014.

[184] S. Kim and S. Member, "Deep Reinforcement Learning-Based Traffic Sampling for Multiple Traffic Analyzers on Software-Defined Networks," IEEE Access, vol. 9, pp. 47815-47827, 2021.

[185] T. A. Tang, L. Mhamdi, D. McLernon, S. A. R. Zaidi, and M. Ghogho, "Deep learning approach for Network Intrusion Detection in Software Defined Networking," in International Conference on Wireless Networks and Mobile Communications, WINCOM 2016: Green Communications and Networking, 2016, pp. 258-263.

[186] T. A. Tang, L. Mhamdi, D. McLernon, S. A. R. Zaidi, and M. Ghogho, "Deep Recurrent Neural Network for Intrusion Detection in SDNbased Networks," in 4th IEEE Conference on Network Softwarization and Workshops, NetSoft, 2018, pp. 462-469.

[187] S. Maeda, A. Kanai, S. Tanimoto, T. Hatashima, and K. Ohkubo, "A Botnet Detection Method on SDN using Deep Learning," in IEEE International Conference on Consumer Electronics, ICCE, 2019, pp. 16.

[188] T. A. Tang, L. Mhamdi, D. McLernon, S. A. R. Zaidi, M. Ghogho, and F. El Moussa, "DeepIDS: Deep learning approach for intrusion detection in software defined networking," Electron., vol. 9, no. 9, pp. $1-18,2020$

[189] M. A. Albahar, "Recurrent Neural Network Model Based on a New Regularization Technique for Real-Time Intrusion Detection in SDN Environments," Secur. Commun. Networks, 2019.

[190] L. Chuanhuang, W. Yan, Y. Xiaoyong, S. Zhengjun, and W. Weiming, "Detection and defense of DDoS attack-based on deep learning in OpenFlow-based SDN," Int. J. Commun. Syst., vol. 31, no. 5, pp. 115, 2017.

[191] S. Boukria and M. Guerroumi, "Intrusion detection system for SDN network using deep learning approach," in International Conference on Theoretical and Applicative Aspects of Computer Science, ICTAACS, 2019 , pp. 1-6.

[192] "Open Networking Foundation. 2021. Open Network Operating 
System (ONOS) SDN Controller for SDN/NFV Solutions. [online]." [Online]. Available: https://opennetworking.org/onos/. [Accessed: 15Feb-2021]

[193] I. Letteri, G. Della Penna, and G. De Gasperis, "Botnet detection in software defined networks by deep learning techniques," in International Symposium on Cyberspace Safety and Security, 2018, pp. $49-62$.

[194] I. I. Kurochkin and S. S. Volkov, "Using GRU based deep neural network for intrusion detection in software-defined networks," IOP Conf. Ser. Mater. Sci. Eng., vol. 927, no. 1, 2020.

[195] M. P. Novaes, L. F. Carvalho, J. Lloret, and M. L. Proenca, "Long short-term memory and fuzzy logic for anomaly detection and mitigation in software-defined network environment," IEEE Access, vol. 8, pp. 83765-83781, 2020.

[196] J. D. Gadze, A. A. Bamfo-Asante, J. O. Agyemang, H. Nunoo-Mensah, and K. A.-B. Opare, "An Investigation into the Application of Deep Learning in the Detection and Mitigation of DDOS Attack on SDN Controllers," Technologies, vol. 9, no. 1, p. 14, 2021.

[197] A. Ali and M. M. Yousaf, "Novel Three-Tier Intrusion Detection and Prevention System in Software Defined Network," IEEE Access, vol. 8, pp. 109662-109676, 2020.

[198] J. Kim, J. Kim, H. Kim, M. Shim, and E. Choi, "CNN-based network intrusion detection against denial-of-service attacks," Electron., vol. 9, no. 6 , pp. $1-21,2020$.

[199] S. K. Dey and R. M. Mahbubur, "Effects of Machine Learning Approach in Flow-Based Anomaly Detection on Software-Defined Networking," Symmetry (Basel)., vol. 12, no. 7, pp. 1-21, 2019.

[200] P. Baldi, "Autoencoders, Unsupervised Learning, and Deep Architectures," ICML Unsupervised Transf. Learn., pp. 37-50, 2012.

[201] F. Asja and I. Christian, "An introduction to restricted Boltzmann machines," in CIARP 2012: Progress in Pattern Recognition, Image Analysis, Computer Vision, and Applications, 2012, pp. 14-36.

[202] Q. Niyaz, W. Sun, and A. Y. Javaid, "A Deep Learning Based DDoS Detection System in Software-Defined Networking (SDN)," ICST Trans. Secur. Saf., vol. 4, no. 12, p. 153515, 2017.

[203] R. M. A. Ujjan, Z. Pervez, K. Dahal, A. K. Bashir, R. Mumtaz, and J. González, "Towards sFlow and adaptive polling sampling for deep learning based DDoS detection in SDN," Futur. Gener. Comput. Syst., vol. 111, pp. 763-779, 2020.

[204] A. Dawoud, S. Shahristani, and C. Raun, "Unsupervised Deep Learning for Software Defined Networks Anomalies Detection," Trans. Comput. Collect. Intell. XXXIII. Springer, Berlin, Heidelberg, 2019. 167-178., vol. 11610, pp. 167-178, 2019.

[205] B. Han and X. Yang, "OverWatch: A Cross-Plane DDoS Attack Defense Framework with Collaborative Intelligence in SDN," Secur. Commun. Networks, vol. 2018, 2018.

[206] M. P. Novaes, L. F. Carvalho, J. Lloret, and M. L. Proença, "Adversarial Deep Learning approach detection and defense against DDoS attacks in SDN environments," Futur. Gener. Comput. Syst., vol. 125, pp. 156-167, 2021.

[207] B. Hartpence, "The RIT SDN Testbed and GENI," Rochester Inst. Technol., 2015.

[208] J. Shu, L. Zhou, W. Zhang, X. Du, and M. Guizani, "Collaborative Intrusion Detection for VANETs: A Deep Learning-Based Distributed SDN Approach,” IEEE Trans. Intell. Transp. Syst., vol. 22, no. 7, pp. 4519-4530, 2021.

[209] P. Mohanapriya and S. M. Shalinie, "Restricted Boltzmann Machine based detection system for DDoS attack in Software Defined Networks," in 4th International Conference on Signal Processing, Communication and Networking, ICSCN, 2017, pp. 14-19.

[210] "Controller Benchmark-Cbench," 2013. [Online]. Available: https://floodlight.atlassian.net/wiki/spaces/floodlightcontroller/pages/ 1343657/Cbench+New.

[211] D. Erickson, "The Beacon OpenFlow controller," HotSDN 2013 Proc. 2013 ACM SIGCOMM Work. Hot Top. Softw. Defin. Netw., pp. $13-18,2013$.

[212] “CIC-DDOS 2019." [Online]. Available: https://www.unb.ca/cic/datasets/ddos-2019.html. [Accessed: 20-Feb2020].

[213] I. Sharafaldin, A. H. Lashkari, S. Hakak, and A. A. Ghorbani, "Developing realistic distributed denial of service (DDoS) attack dataset and taxonomy," in International Carnahan Conference on Security Technology (ICCST),IEEE., 2019, pp. 1-8.

[214] T. H. Lee, L. H. Chang, and C. W. Syu, "Deep learning enabled intrusion detection and prevention system over SDN networks," in
IEEE International Conference on Communications Workshops, ICC Workshops, 2020, pp. 2-7.

[215] M. Berman et al., "GENI: A federated testbed for innovative network experiments," Comput. Networks, vol. 61, no. 2014, pp. 5-23, 2014.

[216] A. AlEroud and G. Karabatis, "SDN-GAN: Generative adversarial deep NNs for synthesizing cyber attacks on software defined networks," in OTM Confederated International Conferences" On the Move to Meaningful Internet Systems". Springer, Cham, 2019., 2019, vol. 11878 LNCS, pp. 211-220.

[217] M. V. O. Assis, L. F. Carvalho, J. Lloret, and M. L. Proença, "A GRU deep learning system against attacks in software defined networks," J. Netw. Comput. Appl., vol. 177, p. 102942, 2021.

[218] M. Abdallah, N.-A. Le-Khac, H. Jahromi, and A. Delia Jurcut, "A Hybrid CNN-LSTM Based Approach for Anomaly Detection Systems in SDNs," in 6th International Conference on Availability, Reliability and Security, 2021, pp. 1-7.

[219] M. S. Elsayed, N. A. Le-Khac, and A. D. Jurcut, "InSDN: A novel SDN intrusion dataset," IEEE Access, vol. 8, pp. 165263-165284, 2020.

[220] M. Latah and L. Toker, "An efficient flow-based multi-level hybrid intrusion detection system for software-defined networks," CCF Trans. Netw., vol. 3, no. 3, pp. 261-267, 2019.

[221] J. Malik, A. Akhunzada, I. Bibi, M. Imran, A. Musaddiq, and S. W. Kim, "Hybrid Deep Learning: An Efficient Reconnaissance and Surveillance Detection Mechanism in SDN," IEEE Access, vol. 8, pp. 134695-134706, 2020

[222] A. Vaswani et al., "Attention Is All You Need," in 31st Conference on Neural Information Processing Systems (NIPS 2017), 2017, pp. 59986008 .

[223] H. Y. He, Z. G. Yang, and X. N. Chen, "PERT: Payload Encoding Representation from Transformer for Encrypted Traffic Classification," in ITU Kaleidoscope: Industry-Driven Digital Transformation (ITU K), 2020.

[224] R. F. Bikmukhamedo and A. F. Nadeev, "Generative transformer framework for network traffic generation and classification," T-Comm, vol. 14 , no. 11, pp. 64-71, 2020.

[225] H. Wang and W. Li, "DDosTC: A transformer-based network attack detection hybrid mechanism in SDN," Sensors (Switzerland), vol. 21, no. 15,2021

[226] T. V. Phan and M. Park, "Efficient Distributed Denial-of-Service Attack Defense in SDN-Based Cloud," IEEE Access, vol. 7, pp. 18701-18714, 2019.

[227] P. Ding, J. Li, L. Wang, M. Wen, and Y. Guan, "HYBRID-CNN: An Efficient Scheme for Abnormal Flow Detection in the SDN-Based Smart Grid," Secur. Commun. Networks, vol. 2020, 2020.

[228] M. S. ElSayed, N. A. Le-Khac, M. A. Albahar, and A. Jurcut, "A novel hybrid model for intrusion detection systems in SDNs based on CNN and a new regularization technique," J. Netw. Comput. Appl., vol. 191, no. July, p. 103160, 2021.

[229] S. Garg, N. Kumar, J. J. P. C. Rodrigues, and J. J. P. C. Rodrigues, "Hybrid deep-learning-based anomaly detection scheme for suspicious flow detection in SDN: A social multimedia perspective," IEEE Trans. Multimed., vol. 21, no. 3, pp. 566-578, 2019.

[230] S. Khan and A. Akhunzada, "A hybrid DL-driven intelligent SDNenabled malware detection framework for Internet of Medical Things (IoMT)," Comput. Commun., vol. 170, no. February, pp. 209-216, 2021.

[231] L. Jiaqi, Z. Zhifeng, and L. Rongpeng, "Machine learning-based IDS for software-defined 5G network," IET Networks, vol. 7, no. 2, pp. 5360, 2017.

[232] V. Deepa, K. M. Sudar, and P. Deepalakshmi, "Design of Ensemble Learning Methods for DDoS Detection in SDN Environment," in International Conference on Vision Towards Emerging Trends in Communication and Networking (ViTECoN), 2019, pp. 1-6.

[233] D. Javeed, T. Gao, and M. T. Khan, "Sdn-enabled hybrid dl-driven framework for the detection of emerging cyber threats in iot," Electron., vol. 10, no. 8, pp. 1-16, 2021.

[234] L. Tan, Y. Pan, J. Wu, J. Zhou, H. Jiang, and Y. Deng, "A New Framework for DDoS Attack Detection and Defense in SDN Environment," IEEE Access, vol. 8, pp. 161908-161919, 2020.

[235] K. Muthamil Sudar and P. Deepalakshmi, "A two level security mechanism to detect a DDoS flooding attack in software-defined networks using entropy-based and C4.5 technique," J. High Speed Networks, vol. 26, no. 1, pp. 55-76, 2020.

[236] D. Opitz and R. Maclin, "Popular Ensemble Methods: An Empirical Study,” J. Artif. Intell. Res., vol. 11, pp. 169-198, 1999. 
[237] G. Biau, “Analysis of a random forests model,” J. Mach. Learn. Res., vol. 13, no. 1, pp. 1063-1095., 2013.

[238] Peter Bühlmann, "Bagging, Boosting and Ensemble Methods," in Handbook of Computational Statistics: Concepts and Methods: Second Edition, Springer, 2012, pp. 985-1022.

[239] D. Ahmed, S. A. Kaan, and A. Pelin, "ProtÉdge- A few-shot ensemble learning approach to software-defined networking-assisted edge security," Emeging Telecommun. Technol., pp. 1-27, 2020.

[240] S. Rochak, D. Mayank, and R. Virender, "Voting-based intrusion detection framework for securing software-defined networks," Concurr. Comput. Pract. Experence., vol. 32, no. 24, pp. 1-16, 2019.

[241] "The CAIDA 'DDoS Attack 2007' Dataset," 2007. [Online]. Available: $\quad$ https://www.caida.org/catalog/datasets/ddos20070804_dataset/. [Accessed: 29-Oct-2021].

[242] "IoT-23 - A labeled dataset with malicious and benign IoT network traffic." [Online]. Available: https://www.stratosphereips.org/datasetsiot23. [Accessed: 29-Oct-2021].

[243] S. Haider, A. Akhunzada, G. Ahmed, and M. Raza, "Deep Learning based Ensemble Convolutional Neural Network Solution for Distributed Denial of Service Detection in SDNs," in UK/China Emerging Technologies, UCET 2019, 2019, pp. 1-4.

[244] S. Zwane, P. Tarwireyi, and M. Adigun, "Ensemble Learning Approach for Flow-based Intrusion Detection System," in IEEE AFRICON Conference, 2019, pp. 1-8.

[245] S. Zwane, P. Tarwireyi, and M. Adigun, "A Flow-based IDS for SDNenabled Tactical Networks," in International Multidisciplinary Information Technology and Engineering Conference, IMITEC, 2019.

[246] L. Yang, Y. Song, S. Gao, B. Xiao, and A. Hu, "Griffin: An Ensemble of AutoEncoders for Anomaly Traffic Detection in SDN," in IEEE Conference and Exhibition on Global Telecommunications (GLOBECOM), 2020, pp. 1-6.

[247] Y. Mirsky, T. Doitshman, Y. Elovici, and A. Shabtai, "Kitsune: An ensemble of autoencoders for online network intrusion detection," arXiv Prepr. arXiv1802.09089, no. February, 2018.

[248] Z. Chen, F. Jiang, Y. Cheng, X. Gu, W. Liu, and J. Peng, "XGBoost Classifier for DDoS Attack Detection and Analysis in SDN-Based Cloud," in IEEE International Conference on Big Data and Smart Computing, BigComp, 2018, pp. 251-256.

[249] S. Sen, K. D. Gupta, and M. Manjurul Ahsan, "Leveraging Machine Learning Approach to Setup Software-Defined Network(SDN) Controller Rules During DDoS Attack," in International Joint Conference on Computational Intelligence, 2019, pp. 49-60.

[250] N. Van Thanh, H. Bao, and T. N. Thinh, "An anomaly-based intrusion detection architecture integrated on openflow switch," in ACM International Conference Proceeding Series, 2016, pp. 96-100.

[251] H. A. Alamri and V. Thayananthan, "Bandwidth Control Mechanism and Extreme Gradient Boosting Algorithm for Protecting SoftwareDefined Networks Against DDoS Attacks," IEEE Access, vol. 8, pp. 194269-194288, 2020.

[252] A. O. Alzahrani and M. J. F. Alenazi, "Designing a network intrusion detection system based on machine learning for software defined networks," Futur. Internet, vol. 13, no. 5, 2021.

[253] C. B. Zerbini, L. F. Carvalho, T. Abrão, and M. L. Proença, "Wavelet against random forest for anomaly mitigation in software-defined networking," Appl. Soft Comput. J., vol. 80, pp. 138-153, 2019.

[254] M. Conti, A. Gangwal, and M. S. Gaur, "A comprehensive and effective mechanism for DDoS detection in SDN," in International Conference on Wireless and Mobile Computing, Networking and Communications, 2017, vol. October.

[255] R. F. Fouladi, O. Ermiş, and E. Anarim, "A DDoS attack detection and defense scheme using time-series analysis for SDN," J. Inf. Secur. Appl., vol. 54, no. August, p. 102587, 2020.

[256] D. Yin, L. Zhang, and K. Yang, "A DDoS Attack Detection and Mitigation with Software-Defined Internet of Things Framework," IEEE Access, vol. 6, pp. 24694-24705, 2018.

[257] A. Aleroud and I. Alsmadi, "Identifying DoS attacks on software defined networks: A relation context approach," in Proceedings of the NOMS 2016 - 2016 IEEE/IFIP Network Operations and Management Symposium, 2016, pp. 853-857.

[258] B. Wang, Y. Zheng, W. Lou, and Y. T. Hou, "DDoS attack protection in the era of cloud computing and software-defined networking," in Proceedings - International Conference on Network Protocols, ICNP, 2014, pp. 624-629.

[259] S. Chowdhury et al., "Botnet detection using graph-based feature clustering," J. Big Data, vol. 4, no. 1, 2017.
[260] T. Chin, X. Mountrouidou, X. Li, and K. Xiong, "An SDN-supported collaborative approach for DDoS flooding detection and containment," in Proceedings - IEEE Military Communications Conference MILCOM, 2015, pp. 659-664.

[261] N. Sampath, "Intrusion Detection in Software Defined Networking Using Snort and Mirroring," Int. J. Psychosoc. Rehabil., vol. 23, no. 4, pp. 1699-1710, 2019.

[262] A. Lakhina, M. Crovella, and C. Diot, "Mining anomalies using traffic feature distributions," CACM SIGCOMM Comput. Commun. Rev., vol. 35, no. 4, pp. 217-228, 2005.

[263] Y. Shen, C. Wu, D. Kong, and M. Yang, "TPDD: A Two-Phase DDoS Detection System in Software-Defined Networking," in IEEE International Conference on Communications, 2020, vol. June, p. 107. [264] C. Birkinshaw, E. Rouka, and V. G. Vassilakis, "Implementing an intrusion detection and prevention system using software-defined networking: Defending against port-scanning and denial-of-service attacks," J. Netw. Comput. Appl., vol. 136, pp. 71-85, 2019.

[265] Q. Yan, Q. Gong, and F. A. Deng, "Detection of DDoS attacks against wireless SDN controllers based on the fuzzy synthetic evaluation decision-making model," Ad-Hoc Sens. Wirel. Networks, vol. 33, no. 1-4, pp. 275-299, 2016.

[266] D. Gao, Z. Liu, Y. Liu, C. H. Foh, T. Zhi, and H. C. Chao, "Defending against Packet-In messages flooding attack under SDN context," Soft Comput., vol. 22, no. 20, pp. 6797-6809, 2018.

[267] T. Dang-Van and H. Truong-Thu, "A Multi-Criteria based Software Defined Networking System Architecture for DDoS-Attack Mitigation," REV J. Electron. Commun., vol. 6, no. 3-4, pp. 50-60, 2017.

[268] N. An, I. I. Detection, A. Aleroud, and I. Alsmadi, "Identifying CyberAttacks on Software Defined Networks : An Inference-based Intrusion Detection Approach,” J. Netw. Comput. Appl., 2016.

[269] I. Aliyu, M. C. Feliciano, S. Van Engelenburg, D. O. Kim, and C. G. Lim, "A Blockchain-Based Federated Forest for SDN-Enabled InVehicle Network Intrusion Detection System," IEEE Access, vol. 9, pp. 102593-102608, 2021.

[270] W. Li, J. Tan, and Y. Wang, "A Framework of Blockchain-Based Collaborative Intrusion Detection in Software Defined Networking," in International Conference on Network and System Security., 2020.

[271] I. H. Abdulqadder, S. Zhou, I. T. Aziz, D. Zou, X. Deng, and S. M. Abrar Akber, "An Effective Lightweight Intrusion Detection System with Blockchain to Mitigate Attacks in SDN/NFV Enabled Cloud," in 6th International Conference for Convergence in Technology, I2CT., 2021, pp. 1-8.

[272] J. Kim and S. Shin, "Software-Defined HoneyNet: Towards Mitigating Link Flooding Attacks," in Proceedings - 47th Annual IEEE/IFIP International Conference on Dependable Systems and Networks Workshops, DSN-W 2017, pp. 99-100.

[273] S. Kyung, W. Han, N. Tiwari, V. H. Dixit, and L. Srinivas, "HONEY PROXY : Design and Implementation of Next-Generation Honeynet via SDN," in IEEE Conference on Communications and Network Security (CNS), 2004.

[274] A. M. Zarca, J. B. Bernabe, A. Skarmeta, and J. M. Alcaraz Calero, "Virtual IoT HoneyNets to mitigate cyberattacks in SDN/NFVEnabled IoT networks," IEEE J. Sel. Areas Commun., vol. 38, no. 6, pp. 1262-1277, 2020.

[275] "DARPA1998 Dataset.," 1998. [Online]. Available: http://www.ll.mit.edu/r-d/datasets/1998-darpa-intrusiondetectionevaluation-dataset. [Accessed: 28-Sep-2020].

[276] S. Zwane, P. Tarwireyi, and M. Adigun, "Performance analysis of machine learning classifiers for intrusion detection," in international Conference on Intelligent and Innovative Computing Applications, ICONIC 2018, 2019, pp. 1-5.

[277] "KDD99 Dataset.," 1999. [Online]. Available: http://kdd.ics.uci.edu/databases/kddcup99/kddcup99.html. [Accessed: 28-Sep-2020].

[278] "NSL-KDD99 Dataset.," 2009. [Online]. Available: https://www.unb.ca/cic/datasets/nsl.html. [Accessed: 28-Sep-2020].

[279] N. Moustafa and J. Slay, "UNSW-NB15: A comprehensive data set for network intrusion detection systems (UNSW-NB15 network data set)," 2015 Mil. Commun. Inf. Syst. Conf. MilCIS 2015 - Proc., no. December, 2015.

[280] A. Shiravi, H. Shiravi, M. Tavallaee, and A. A. Ghorbani, "Toward developing a systematic approach to generate benchmark datasets for intrusion detection," Comput. Secur., vol. 31, no. 3, pp. 357-374, 2012. I. Sharafaldin, A. H. Lashkari, and A. A. Ghorbani, "Toward 
generating a new intrusion detection dataset and intrusion traffic characterization," in 4th International Conference on Information Systems Security and Privacy, ICISSP, 2018, pp. 108-116.

[282] R. Panigrahi and S. Borah, "A detailed analysis of CICIDS2017 dataset for designing Intrusion Detection Systems,” Int. J. Eng. Technol., vol. 7, no. 3.24 Special Issue 24, pp. 479-482, 2018.

[283] "CIDDS-001 dataset." [Online]. Available: https://www.hscoburg.de/forschung-kooperation/forschungsprojekteoeffentlich/ingenieurwissenschaften/cidds-coburg-intrusiondetection-data-sets.html. [Accessed: 20-Feb-2021].

[284] M. Ring, S. Wunderlich, D. Grüdl, D. Landes, and A. Hotho, "Flowbased benchmark data sets for intrusion detection," Eur. Conf. Inf. Warf. Secur. ECCWS, pp. 361-369, 2017.

[285] A. Verma and V. Ranga, "Statistical analysis of CIDDS-001 dataset for Network Intrusion Detection Systems using Distance-based Machine Learning," in Procedia Computer Science, 2018, vol. 125, pp. 709716.

[286] "CSE-CIC-IDS2018 on AWS - A collaborative project between the Communications Security Establishment (CSE) \& the Canadian Institute for Cybersecurity (CIC)," 2018. [Online]. Available: https://www.unb.ca/cic/datasets/ids-2018.html. [Accessed: 26-Oct2021].

[287] S. García, M. Grill, J. Stiborek, and A. Zunino, "An empirical comparison of botnet detection methods," Comput. Secur., vol. 45, pp. $100-123,2014$.

[288] "Bro-IDS -An open-source software framework for analyzing network traffic,” 2018. [Online]. Available: https://bricata.com/blog/what-isbro-ids/. [Accessed: 26-Oct-2021].

[289] "CICFlowMeter (ISCXFlowMeter)- A network traffic flow generator and analyser." [Online]. Available: https://www.unb.ca/cic/research/applications.html\#CICFlowMeter.

[Accessed: 26-Oct-2021].

[290] “Argus, Netflow, Flow Tools, Sflow and Jflow." [Online]. Available: https://qosient.com/argus/argusnetflow.shtml. [Accessed: 26-Oct2021].

[291] R. U. Khan, X. Zhang, R. Kumar, A. Sharif, N. A. Golilarz, and M. Alazab, "An adaptive multi-layer botnet detection technique using machine learning classifiers," Appl. Sci., vol. 9, no. 11, 2019.

[292] I. F. Akyildiz, A. Lee, P. Wang, M. Luo, and W. Chou, "Research challenges for traffic engineering in software defined networks," IEEE Netw., vol. 30, no. 3, pp. 52-58, 2016.

[293] Y. Wang, T. Hu, G. Tang, J. Xie, and J. Lu, "SGS: Safe-Guard Scheme for Protecting Control Plane Against DDoS Attacks in SoftwareDefined Networking," IEEE Access, vol. 7, no. 1, pp. 34699-34710, 2019.

[294] T. Hu, Z. Guo, P. Yi, T. Baker, and J. Lan, "Multi-controller Based Software-Defined Networking: A Survey,” IEEE Access, vol. 6, pp. 15980-15996, 2018.

[295] G. Li, X. Wang, and Z. Zhang, "SDN-Based Load Balancing Scheme for Multi-Controller Deployment," IEEE Access, vol. 7, pp. 39612 $39622,2019$.

[296] L. Zhang, Y. Wang, W. Li, X. Qiu, and Q. Zhong, "A survivabilitybased backup approach for controllers in multi-controller SDN against failures," in 19th Asia-Pacific Network Operations and Management Symposium: Managing a World of Things, APNOMS 2017, 2017, pp. 100-105.

[297] S. Scott-Hayward, C. Kane, and S. Sezer, “OperationCheckpoint: SDN application control," in Proceedings - International Conference on Network Protocols, ICNP, 2014, pp. 618-623.

[298] S. Saraswat, V. Agarwal, H. P. Gupta, R. Mishra, A. Gupta, and T. Dutta, "Challenges and solutions in Software Defined Networking: A survey,” J. Netw. Comput. Appl., vol. 141, no. March, pp. 23-58, 2019.

[299] S. Matsumoto, S. Hitz, and A. Perrig, "Fleet: Defending SDNs from malicious administrators," in Proceedings of the third workshop on Hot topics in software defined networking., 2014, pp. 103-108.

[300] D. Kreutz, F. M. V. Ramos, and P. Verissimo, "Towards secure and dependable software-defined networks," in Proceedings of the second ACM SIGCOMM workshop on Hot topics in software defined networking, 2013, no. August, pp. 55-60.

[301] M. Ring, S. Wunderlich, D. Scheuring, D. Landes, and A. Hotho, "A Survey of Network-based Intrusion Detection Data Sets," Comput. Secur., vol. 86, pp. 147-167, 2019.

[302] L. Zhou, M. Liao, C. Yuan, and H. Z. School, "Low-Rate DDoS Attack Detection Using Expectation of Packet Size," Secur. Commun. Networks, 2017.
[303] W. Zhijun, X. Qing, W. Jingjie, Y. Meng, and L. Liang, "Low-Rate DDoS Attack Detection Based on Factorization Machine in Software Defined Network," IEEE Access, vol. 8, pp. 17404-17418, 2020.

[304] W. Chen, S. Xiao, L. Liu, X. Jiang, and Z. Tang, "A DDoS attacks traceback scheme for SDN-based smart city," Comput. Electr. Eng., vol. 81, p. $106503,2020$. 